\title{
Regioselective N-Addition/Substitution Reaction of $\alpha$-Alkylidene Pyrazolinones with Propargyl Sulfonium Salts to Construct Allylthio-Containing Pyrazolones
}

\author{
Shou-Jie Shen, ${ }^{*}, \dagger$ Xiao-Li Du, ${ }^{\dagger}$ Xiao-Li Xu, ${ }^{\ddagger}$ Ming-Gang Zhao,${ }^{\dagger}$ and Jin-Yan Liang $*, \ddagger$ \\ ${ }^{\dagger}$ Key Laboratory of Magnetic Molecules, Magnetic Information Materials Ministry of Education, The \\ School of Chemical and Material Science, Shanxi Normal University, Linfen, 041004, China \\ ${ }^{\star}$ College of Life Science, Shanxi Normal University, Linfen, 041004, China \\ E-mail:shoujie_shen@outlook.com; jinyan_liang@outlook.com
}

Supporting Material
A. X-ray Structure of 3a S2-S3
B. X-ray Structure of 6 S4-S5
C. NMR Spectra .S6-S62 


\section{A. X-ray Structure of 3a}

Crystal of compound 3a was obtained by dissolving product in $n$-Hexane and Ethyl acetate (v/v 5:1) and allowing the solvent to slowly evaporate at room temperature. The single crystal X-ray diffraction study at room temperature revealed that compound $3 \mathbf{a}\left(\mathrm{C}_{27} \mathrm{H}_{24} \mathrm{~N}_{2} \mathrm{OS}\right)$ crystallizes as the centrosymmetric monoclinic space group $\mathrm{P} 21 / \mathrm{c}(Z=4)$ and the crystal structure consists of one crystallographically independent formula unit in the unit cell (Figure S1).
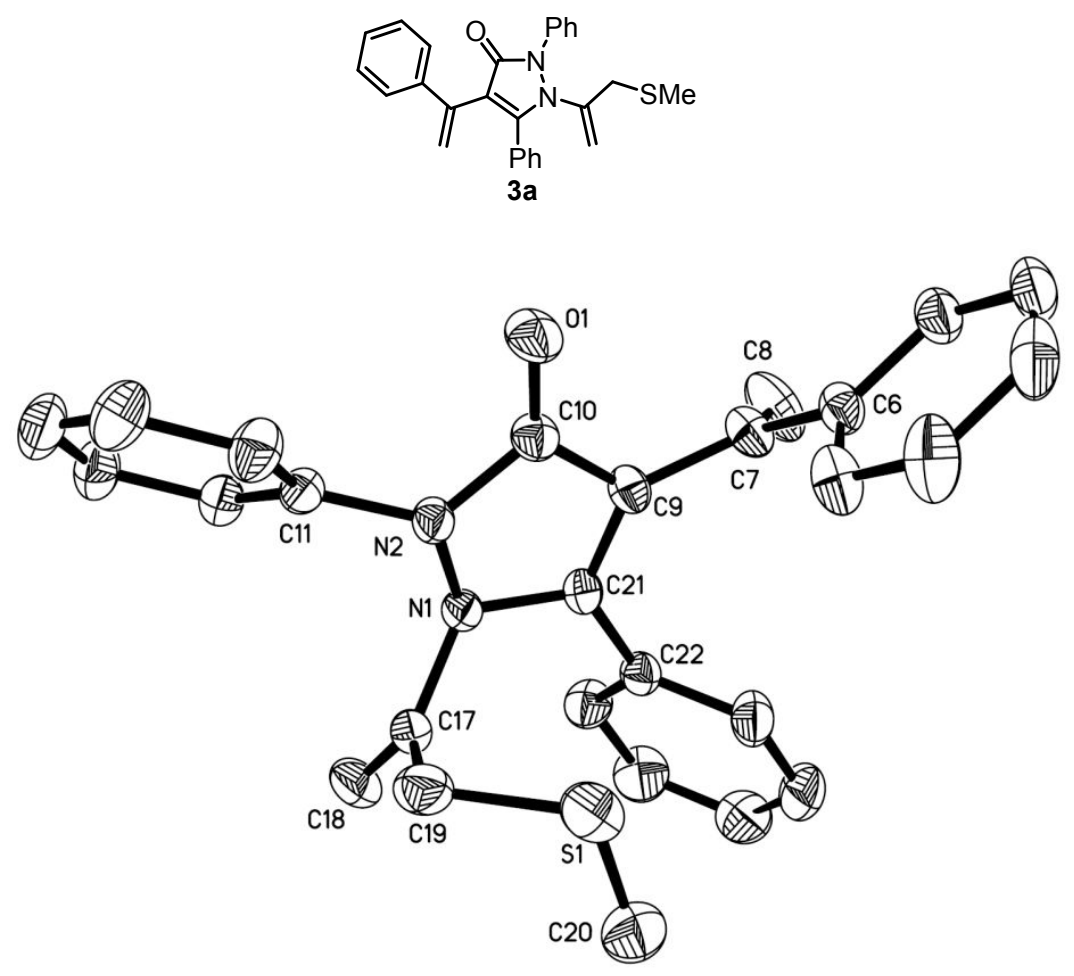

Figure S1. X-ray crystallography structure of 3a. Drawn with ellipsoids at $30 \%$ probability and hydrogen atoms omitted for clarity. 
Table S1. X-ray crystallography data of 3a

\begin{tabular}{|c|c|}
\hline Moiety formula & $\mathrm{C}_{27} \mathrm{H}_{24} \mathrm{~N}_{2} \mathrm{OS}$ \\
\hline $\mathrm{Mr}$ & 424.54 \\
\hline Dx & $1.240 \mathrm{~g} / \mathrm{cm}^{-3}$ \\
\hline Wavelength & 0.71073 \\
\hline Cell & $\begin{array}{c}\mathrm{a}=7.3362(3) \text { alpha }=90 \\
\mathrm{~b}=12.5626(4) \quad \text { beta }=96.443(3) \\
\mathrm{c}=24.8378(10) \text { gamma }=90\end{array}$ \\
\hline Temperature & $293 \mathrm{~K}$ \\
\hline Volume & $2274.64(15)$ \\
\hline Space group & P 21/c \\
\hline Hall group & $-P 2 y b c$ \\
\hline $\mathrm{Z}$ & 4 \\
\hline $\mathrm{Mu}$ & $0.163 / \mathrm{mm}^{-1}$ \\
\hline F000/F000' & $896.0 / 896.81$ \\
\hline $\mathrm{T}_{\min }, \mathrm{T}_{\max }, \mathrm{T}_{\min }$, & $0.976,0.981,0.976$ \\
\hline Data completeness & 1.004 \\
\hline Theta (max) & 26.343 \\
\hline $\mathrm{R}$ (reflections) & $0.0527(3038)$ \\
\hline $\mathrm{wR}_{2}$ (reflections) & $0.1361(4642)$ \\
\hline $\mathrm{S}$ & 1.055 \\
\hline Npar & 297 \\
\hline
\end{tabular}




\section{B. X-ray Structure of 6}

Crystal of compound 6 was obtained by dissolving product in $n$-Hexane and Ethyl acetate (v/v 3:1) and allowing the solvent to slowly evaporate at room temperature. The single crystal X-ray diffraction study at room temperature revealed that compound $\mathbf{6}\left(\mathrm{C}_{26} \mathrm{H}_{21} \mathrm{~N}_{2} \mathrm{OBr}\right)$ crystallizes as the centrosymmetric monoclinic space group $\mathrm{P} 21 / \mathrm{c}(\mathrm{Z}=4)$ and the crystal structure consists of one crystallographically independent formula unit in the unit cell (Figure S2).
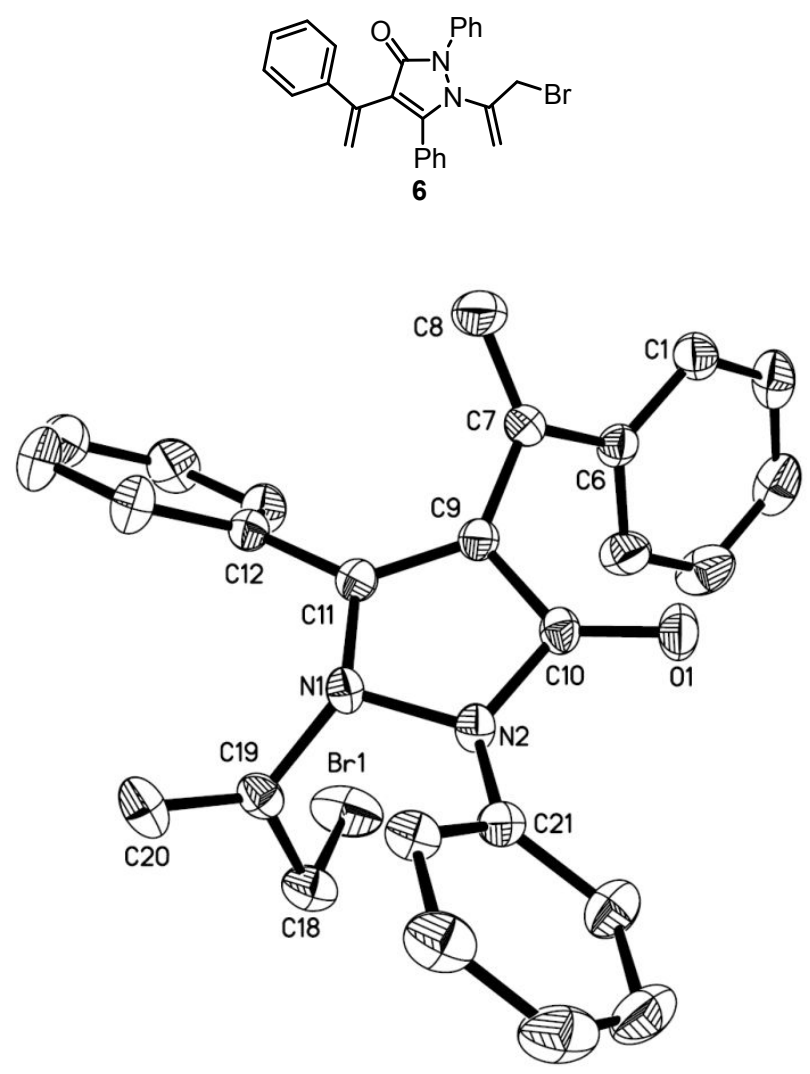

Figure S2. X-ray crystallography structure of 6. Drawn with ellipsoids at 30\% probability and hydrogen atoms omitted for clarity. 
Table S2. X-ray crystallography data of 6

\begin{tabular}{|c|c|}
\hline Moiety formula & $\mathrm{C}_{26} \mathrm{H}_{21} \mathrm{~N}_{2} \mathrm{OBr}$ \\
\hline $\mathrm{Mr}$ & 457.36 \\
\hline Dx & $1.378 \mathrm{~g} / \mathrm{cm}^{-3}$ \\
\hline Wavelength & 0.71073 \\
\hline Cell & $\begin{array}{c}\mathrm{a}=7.0382(2) \text { alpha }=90 \\
\mathrm{~b}=12.4284(4) \quad \text { beta }=95.463(3) \\
\mathrm{c}=25.3081(8) \text { gamma }=90\end{array}$ \\
\hline Temperature & $293 \mathrm{~K}$ \\
\hline Volume & $2203.73(12)$ \\
\hline Space group & P 21/c \\
\hline Hall group & $-\mathrm{P} 2 \mathrm{ybc}$ \\
\hline $\mathrm{Z}$ & 4 \\
\hline $\mathrm{Mu}$ & $1.885 / \mathrm{mm}^{-1}$ \\
\hline F000/F000' & 936.0/935.17 \\
\hline $\mathrm{T}_{\min }, \mathrm{T}_{\max }, \mathrm{T}_{\min }$ & $0.769,1.000,0.666$ \\
\hline Data completeness & 0.905 \\
\hline Theta (max) & 29.340 \\
\hline $\mathrm{R}$ (reflections) & $0.0494(3603)$ \\
\hline $\mathrm{wR}_{2}$ (reflections) & $0.0991(5479)$ \\
\hline $\mathrm{S}$ & 1.024 \\
\hline Npar & 271 \\
\hline
\end{tabular}




\section{NMR Spectra}

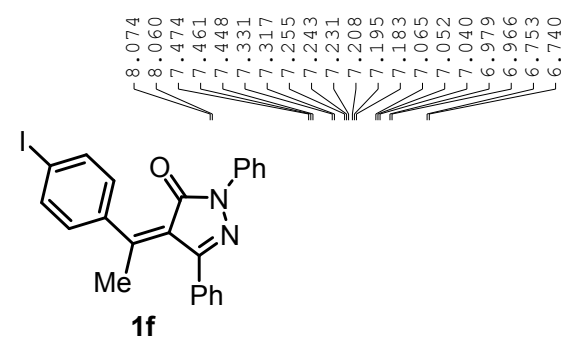

$\mathrm{CDCl}_{3}, 600 \mathrm{MHz}$

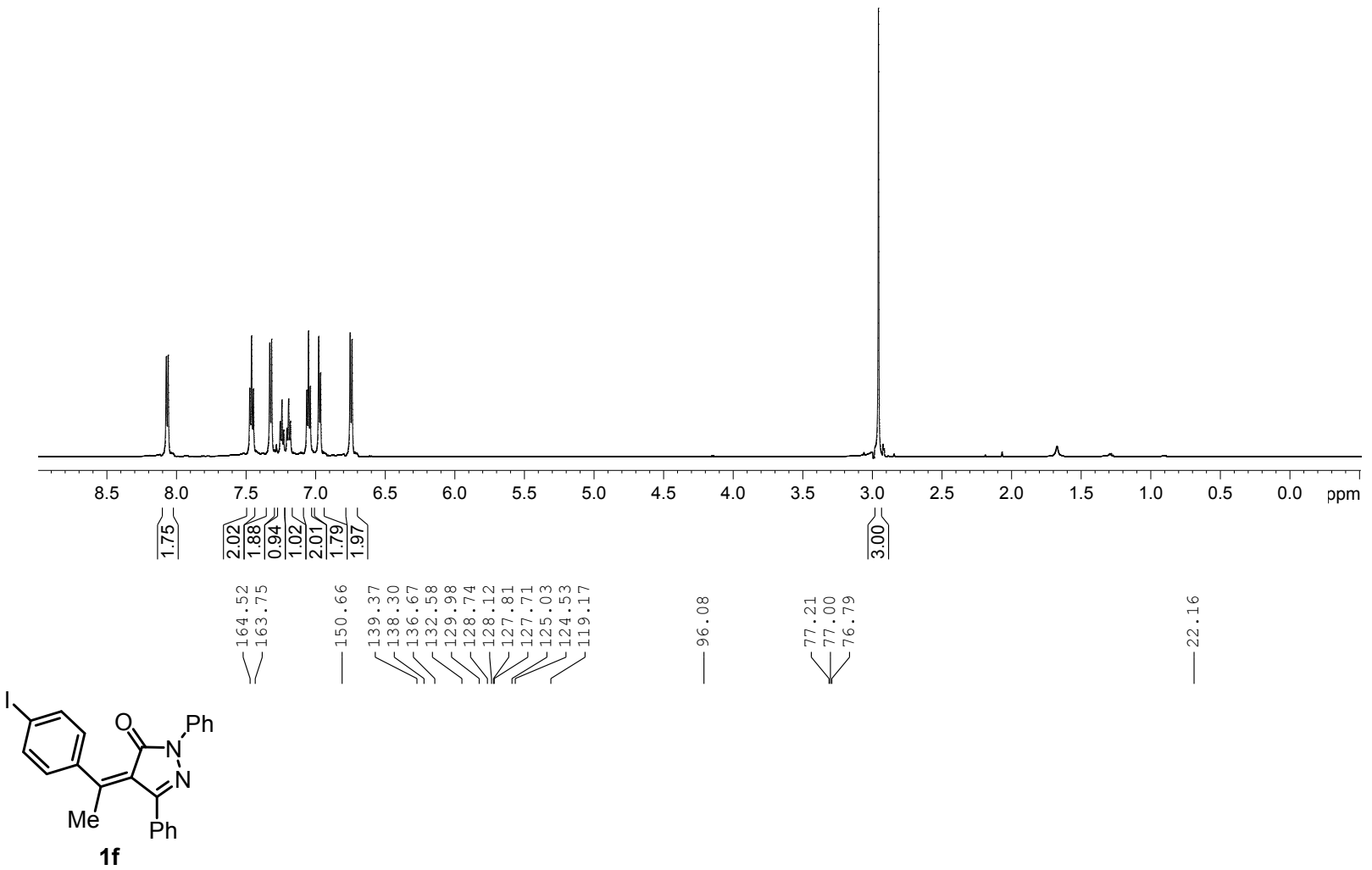

$\mathrm{CDCl}_{3}, 150 \mathrm{MHz}$

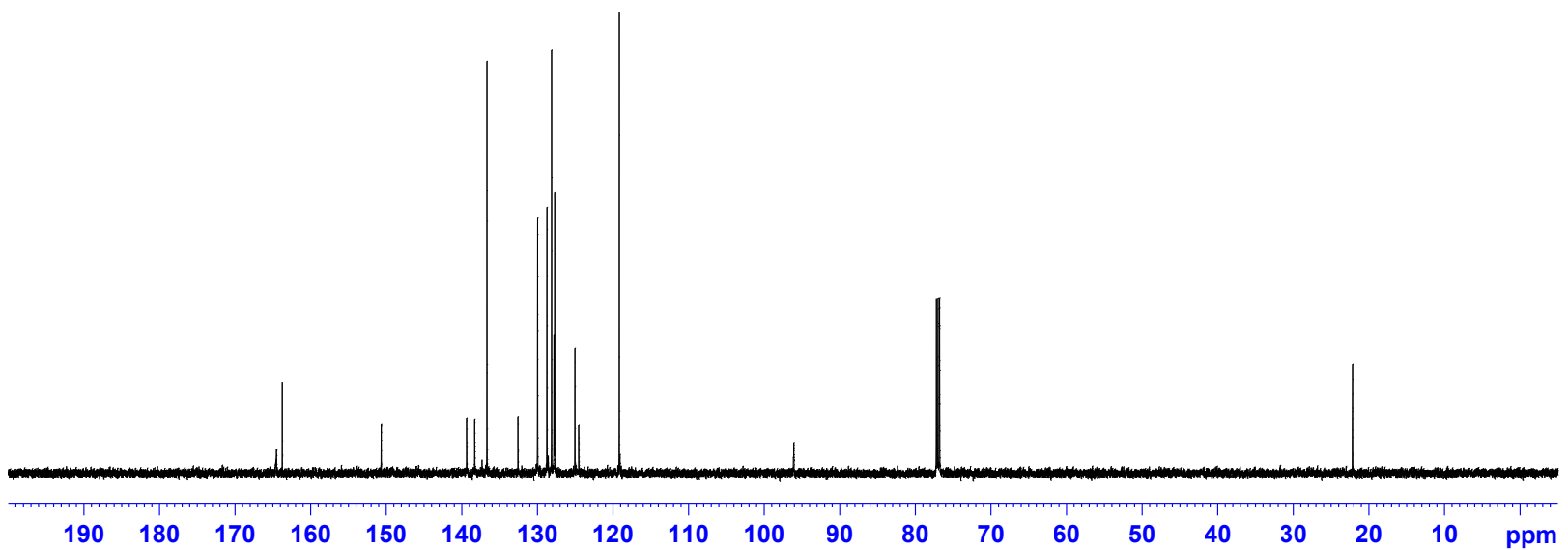



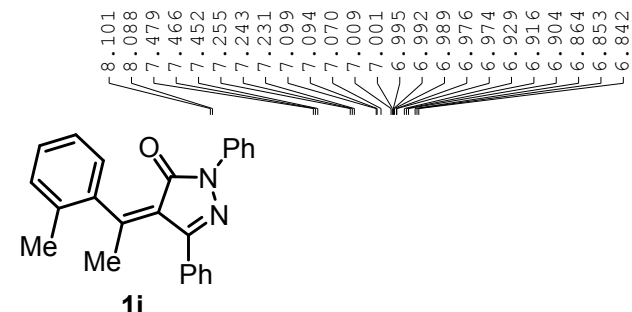

$\mathrm{CDCl}_{3}, 600 \mathrm{MHz}$

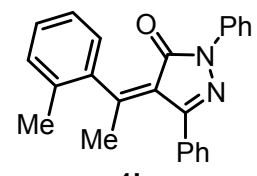

$1 \mathrm{i}$

$\mathrm{CDCl}_{3}, 150 \mathrm{MHz}$

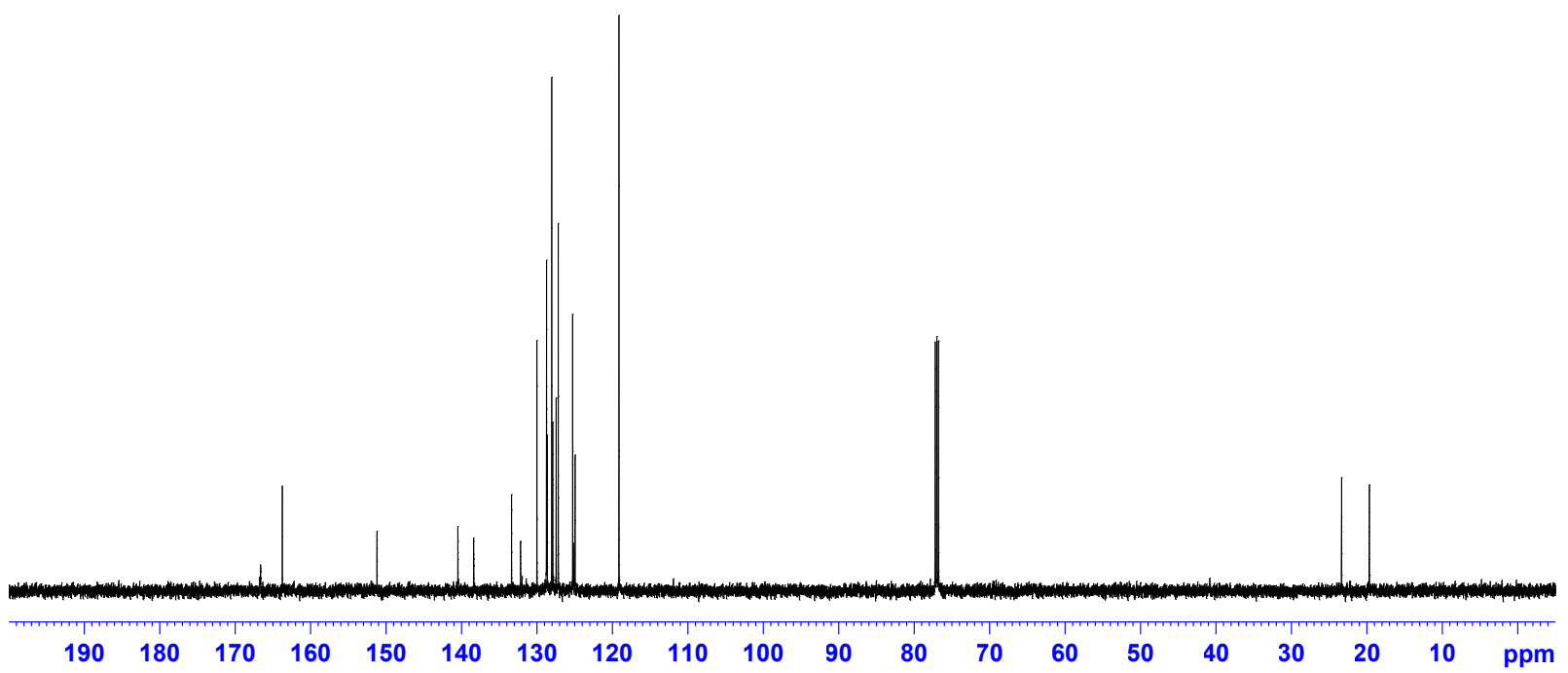




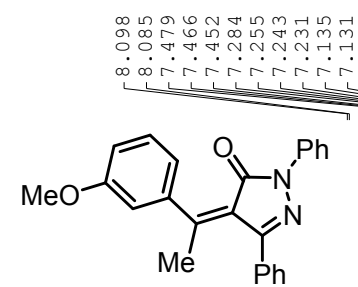

1j

$\mathrm{CDCl}_{3}, 600 \mathrm{MHz}$

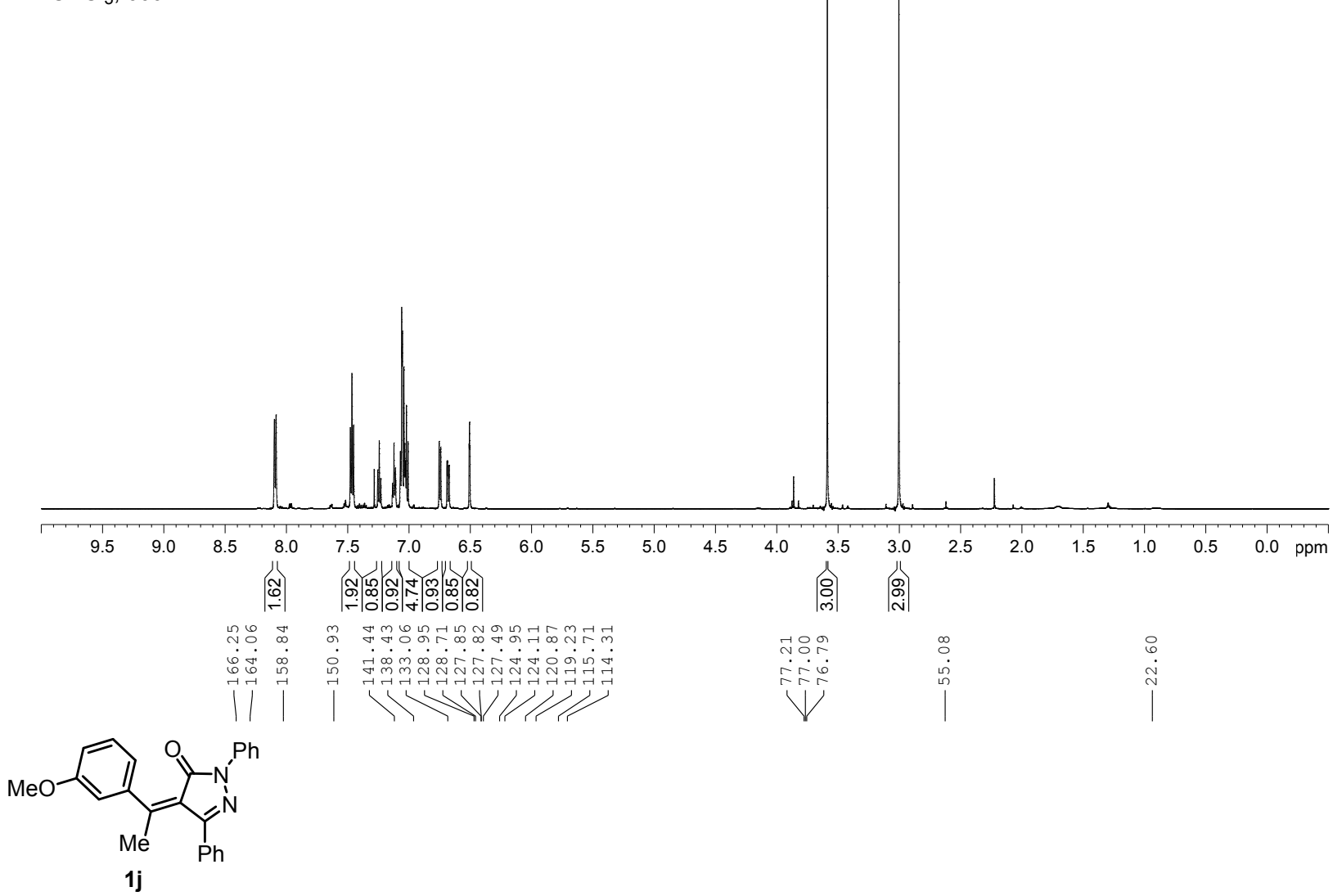

$\mathrm{CDCl}_{3}, 150 \mathrm{MHz}$

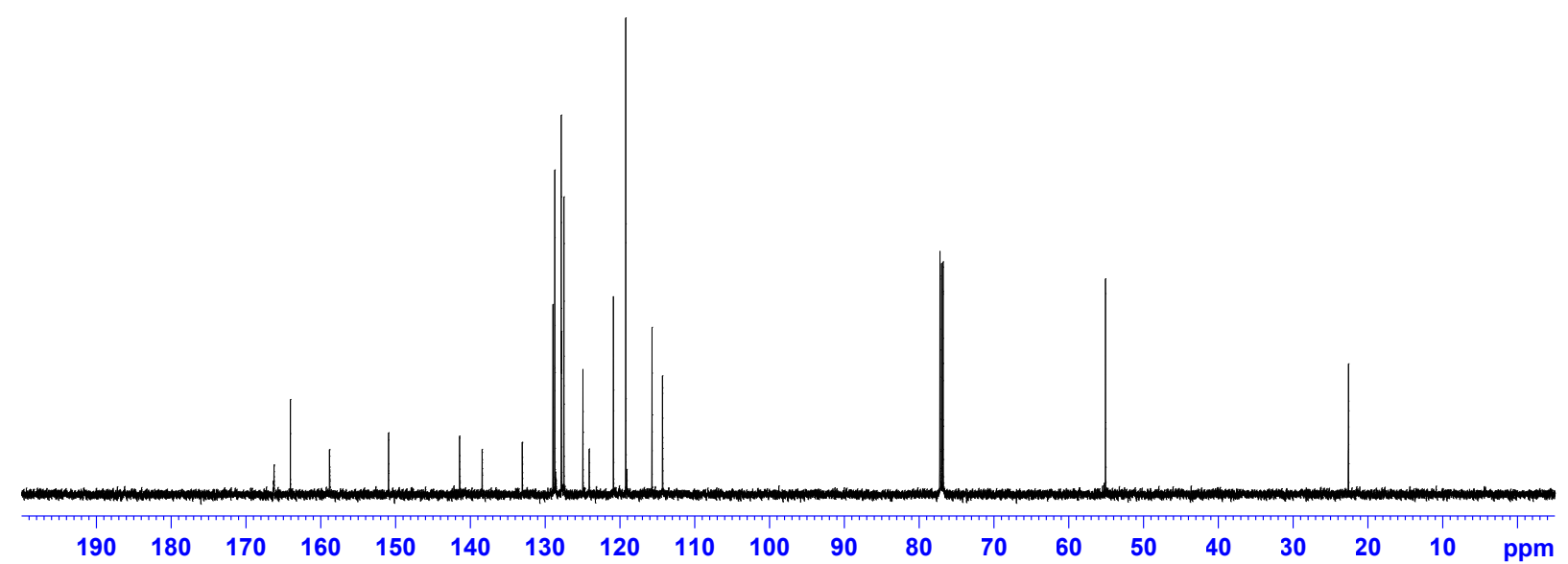




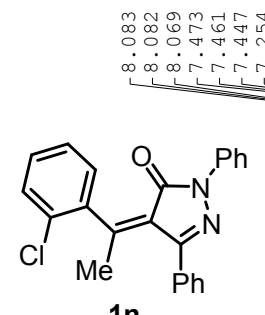

1n

$\mathrm{CDCl}_{3}, 600 \mathrm{MHz}$
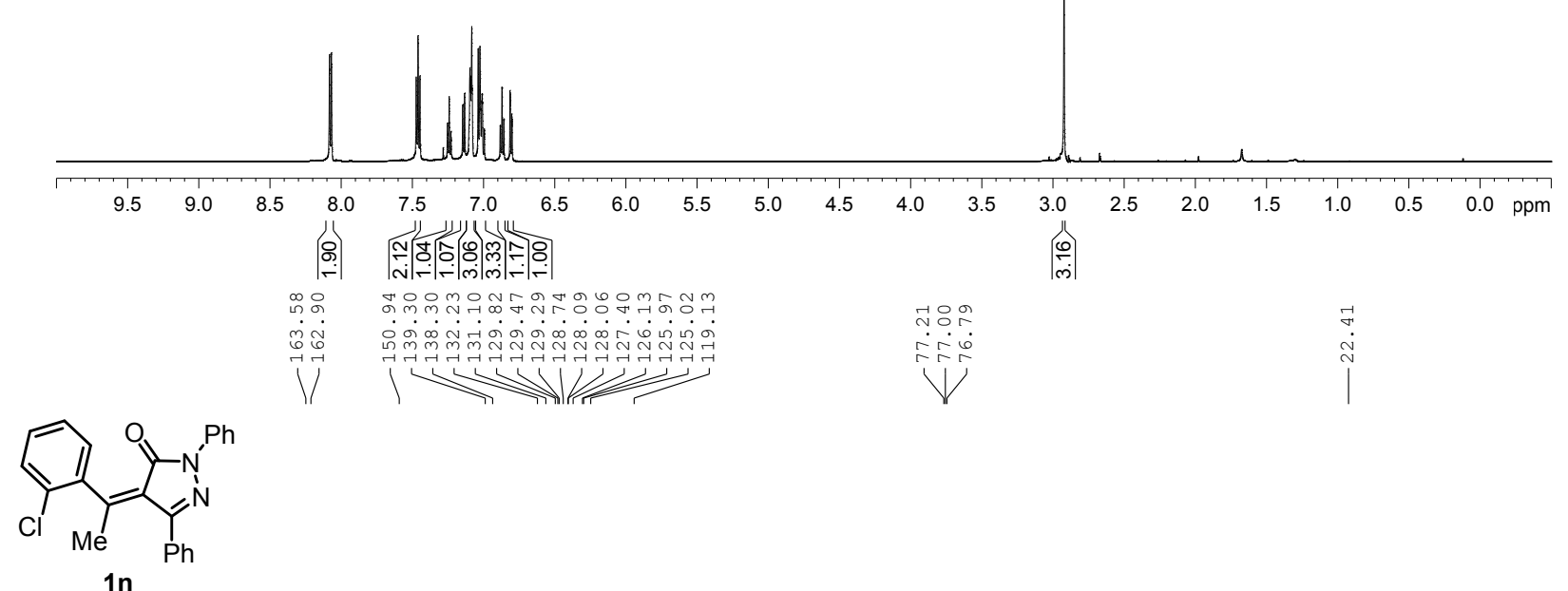

$\mathrm{CDCl}_{3}, 150 \mathrm{MHz}$

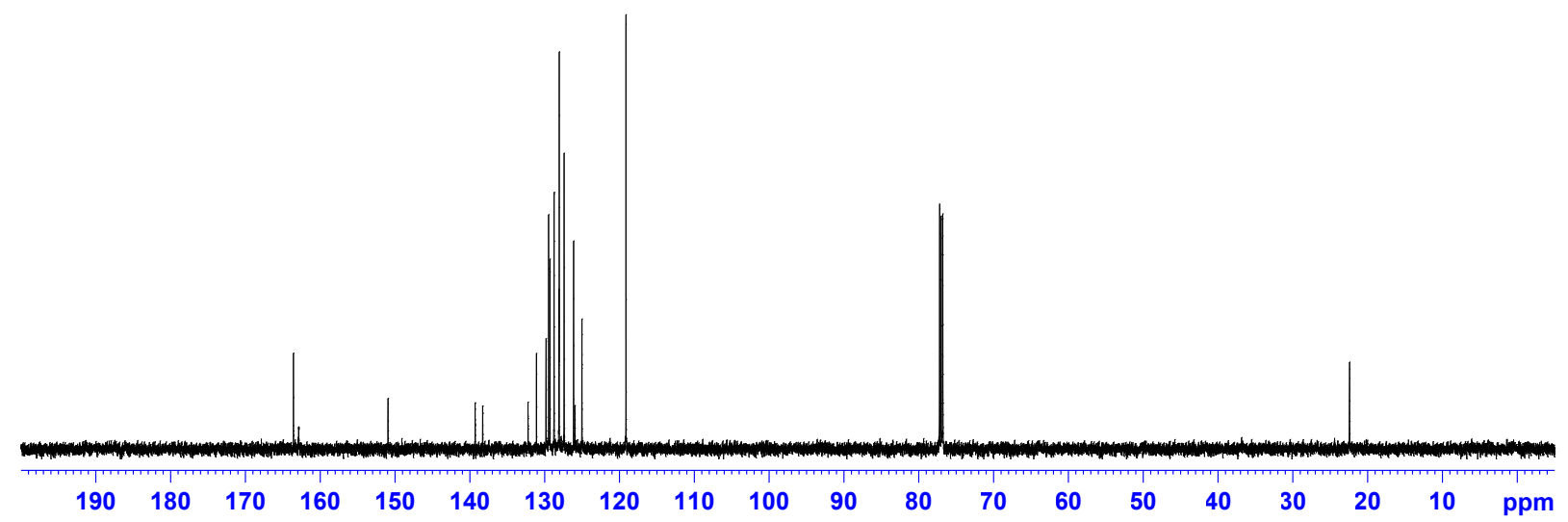




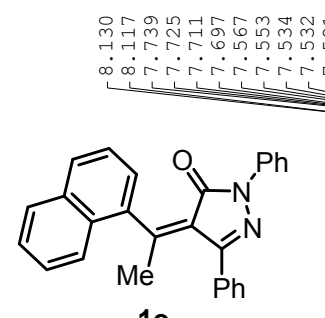

10

$\mathrm{CDCl}_{3}, 600 \mathrm{MHz}$
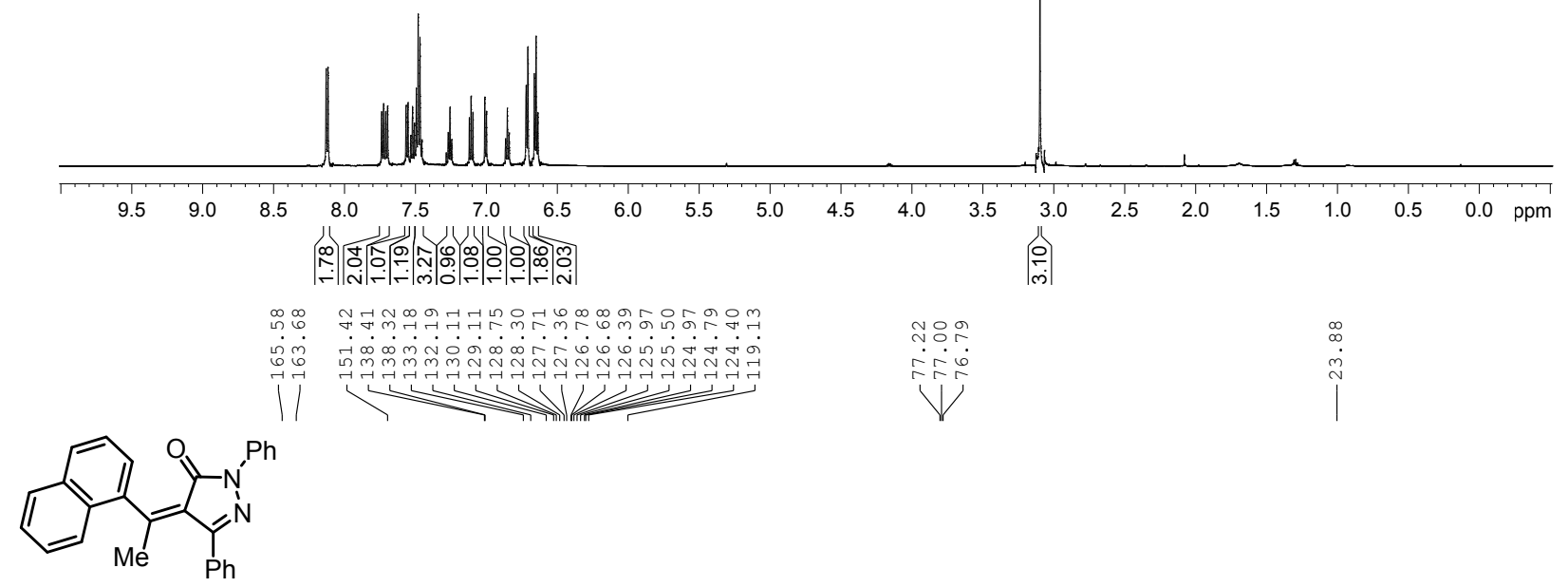

10

$\mathrm{CDCl}_{3}, 150 \mathrm{MHz}$

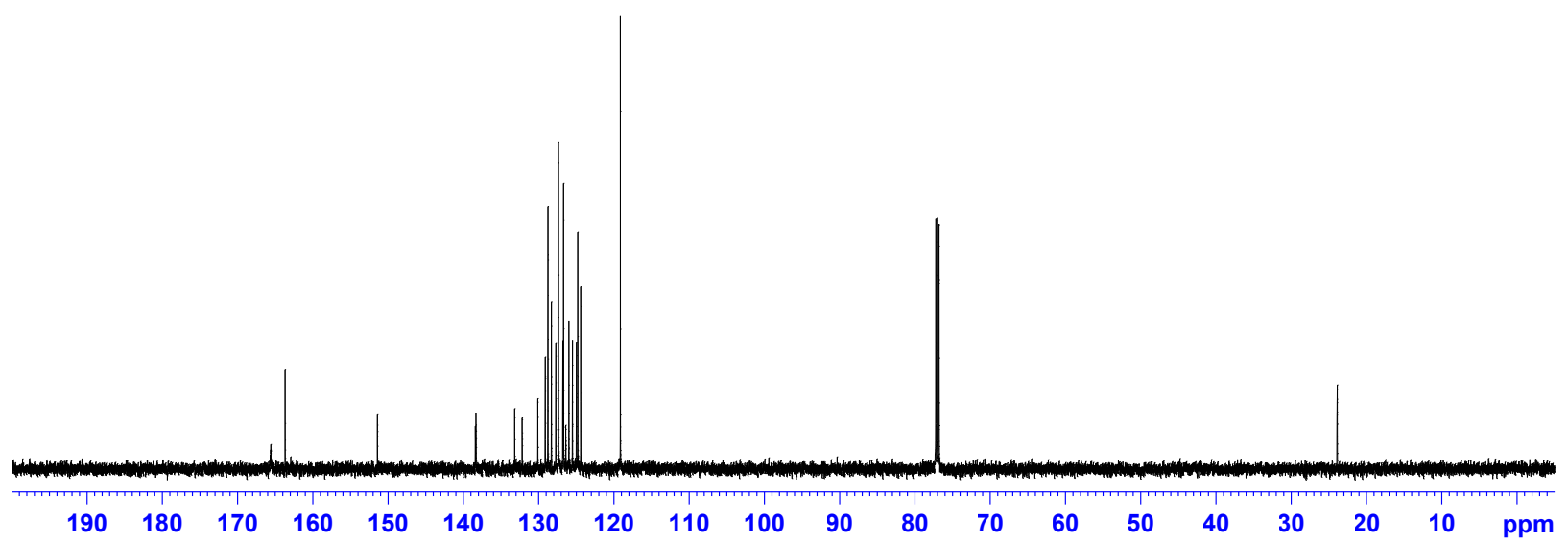




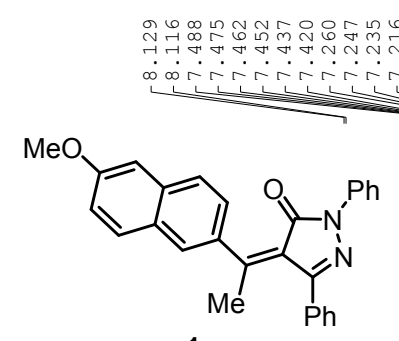

19

$\mathrm{CDCl}_{3}, 600 \mathrm{MHz}$

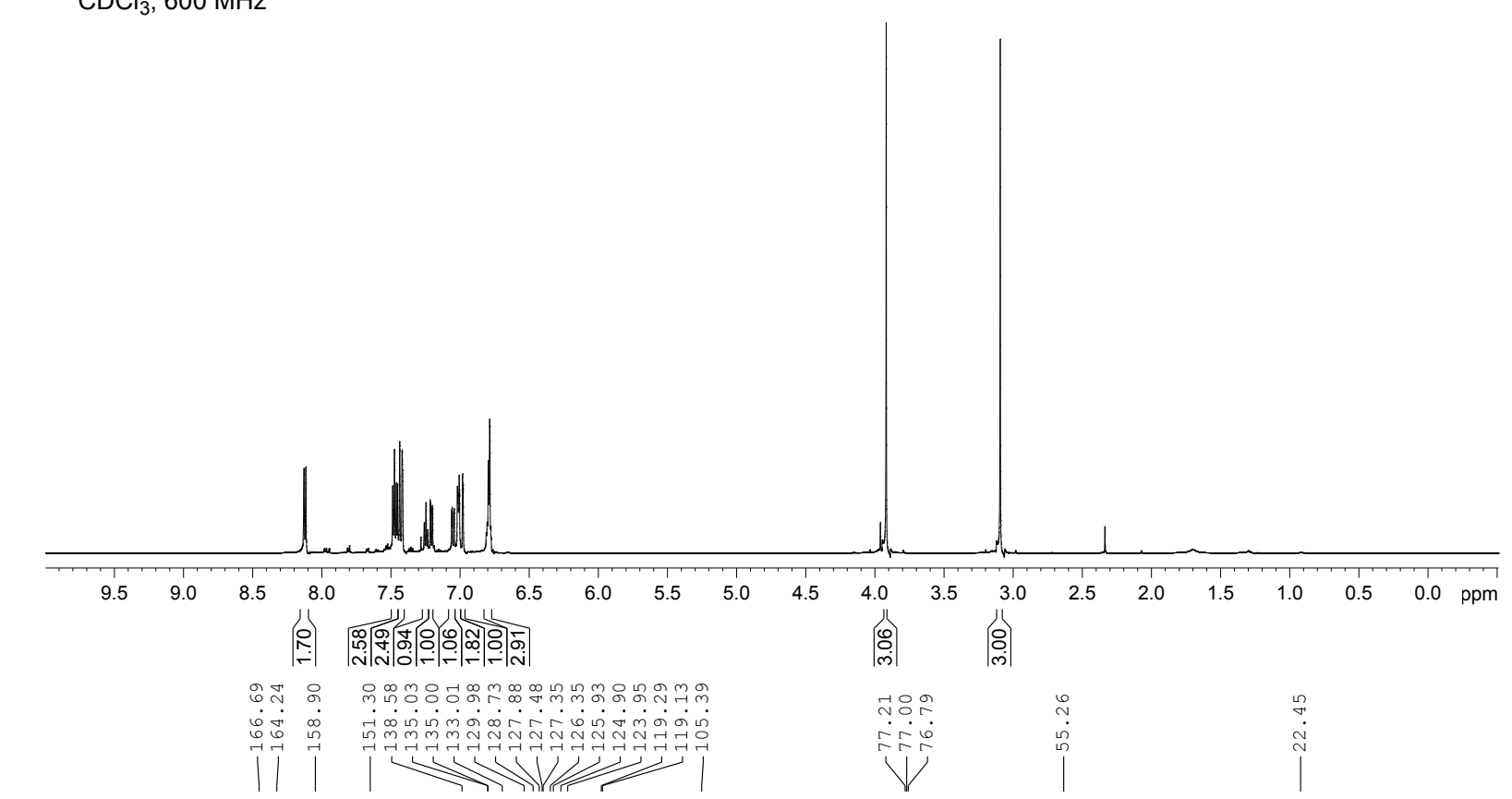<smiles>COc1ccc2cc(/C(C)=C3\C(=O)N(c4ccccc4)N=C3c3ccccc3)ccc2c1</smiles>

19

$\mathrm{CDCl}_{3}, 150 \mathrm{MHz}$

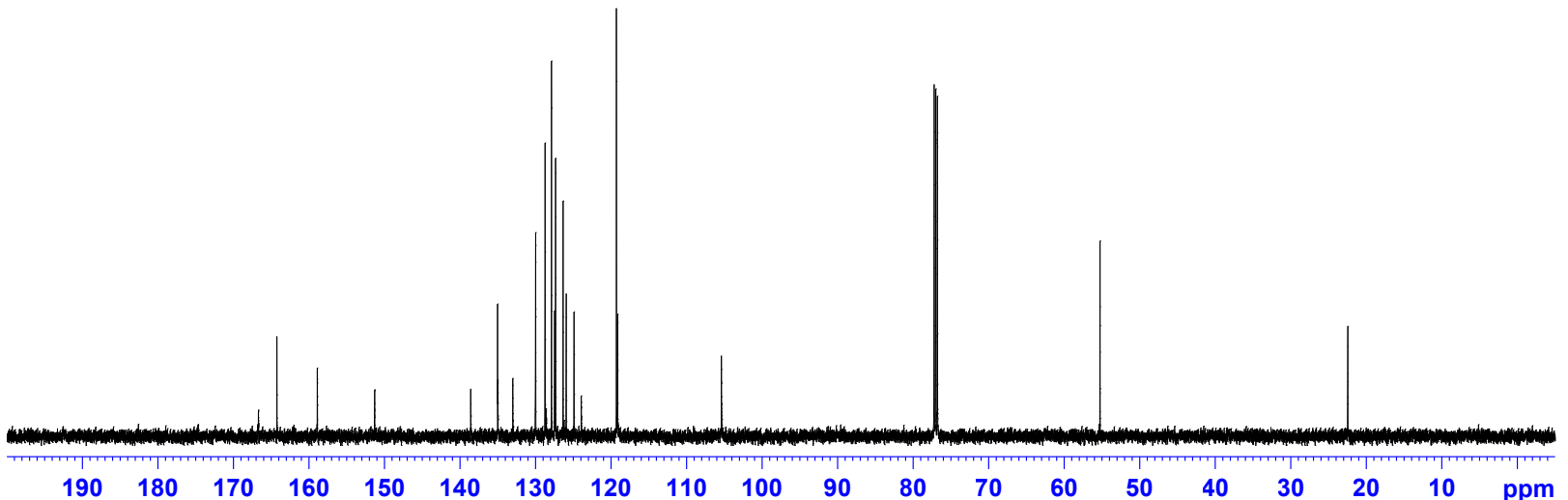



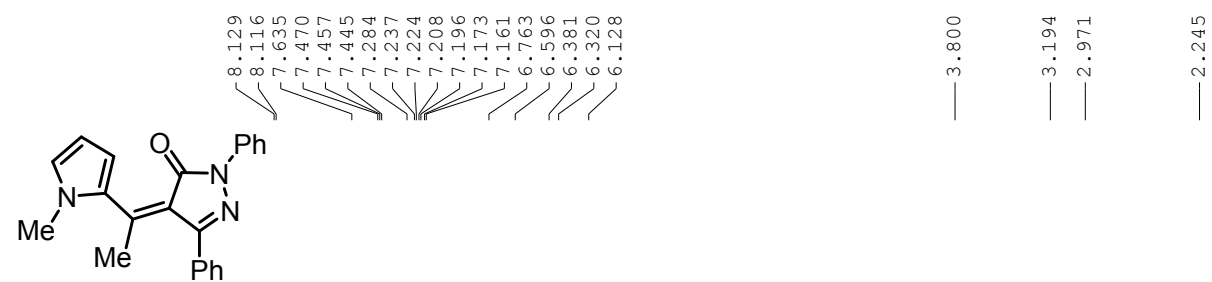

1s (Z:E 6:1)

$\mathrm{CDCl}_{3}, 600 \mathrm{MHz}$
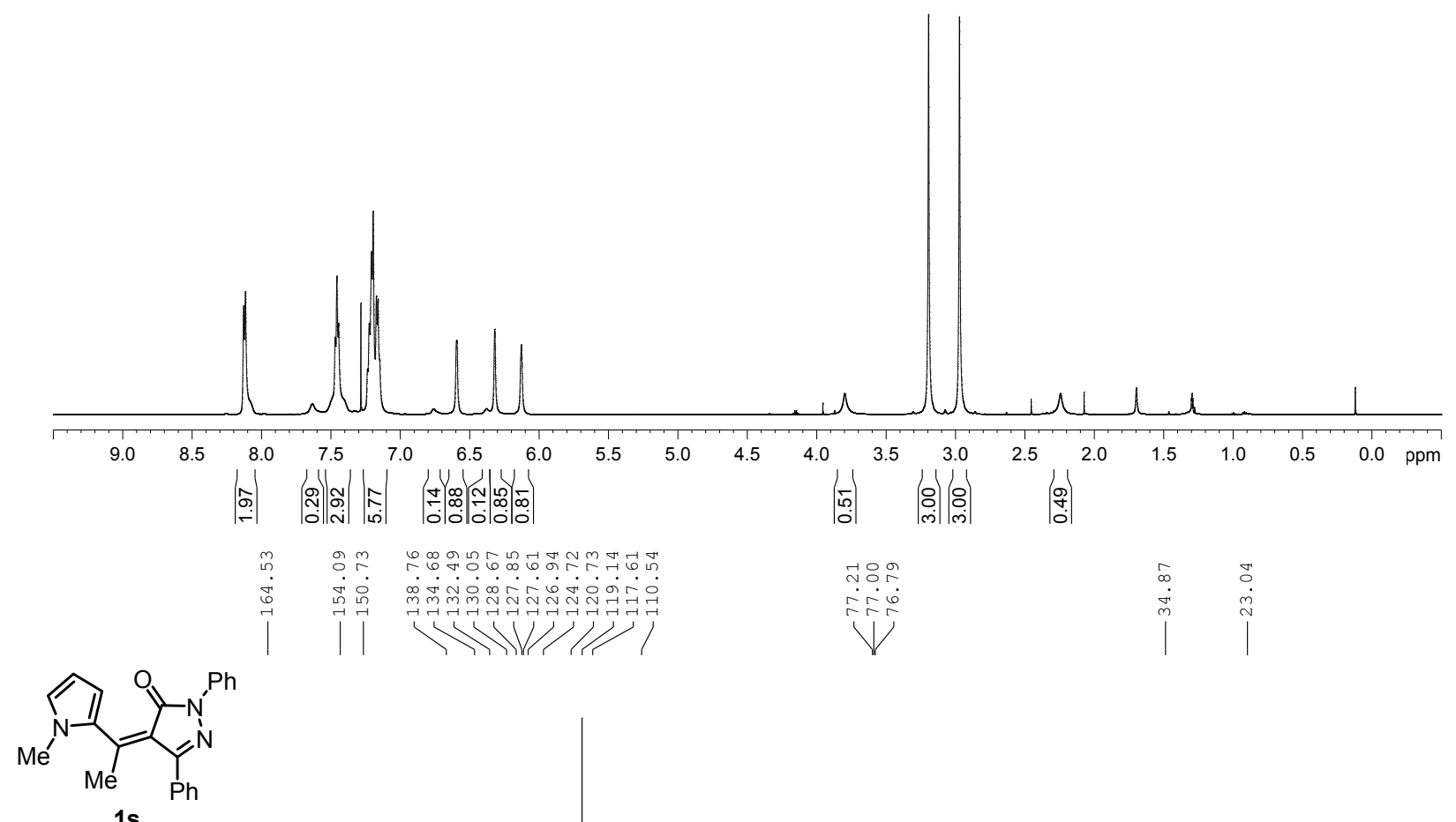

$\mathrm{CDCl}_{3}, 150 \mathrm{MHz}$

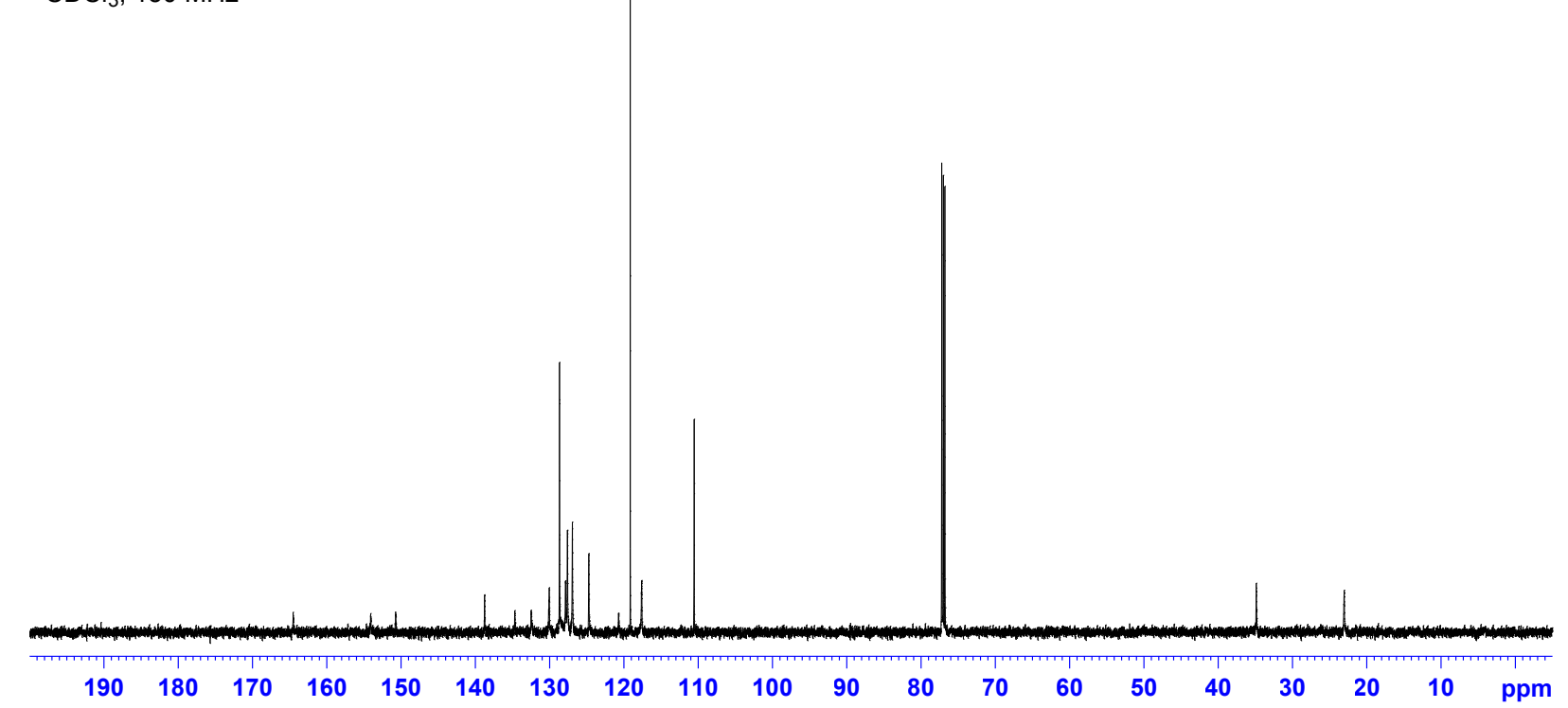




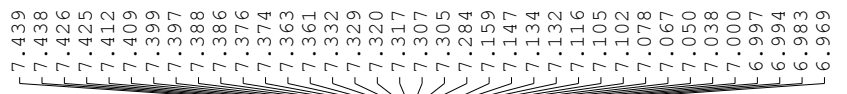<smiles>CCc1ccccc1N1N=C(c2ccccc2)/C(=C(\C)c2ccccc2)C1=O</smiles>

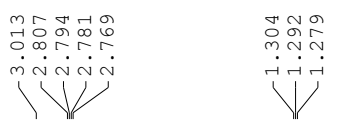

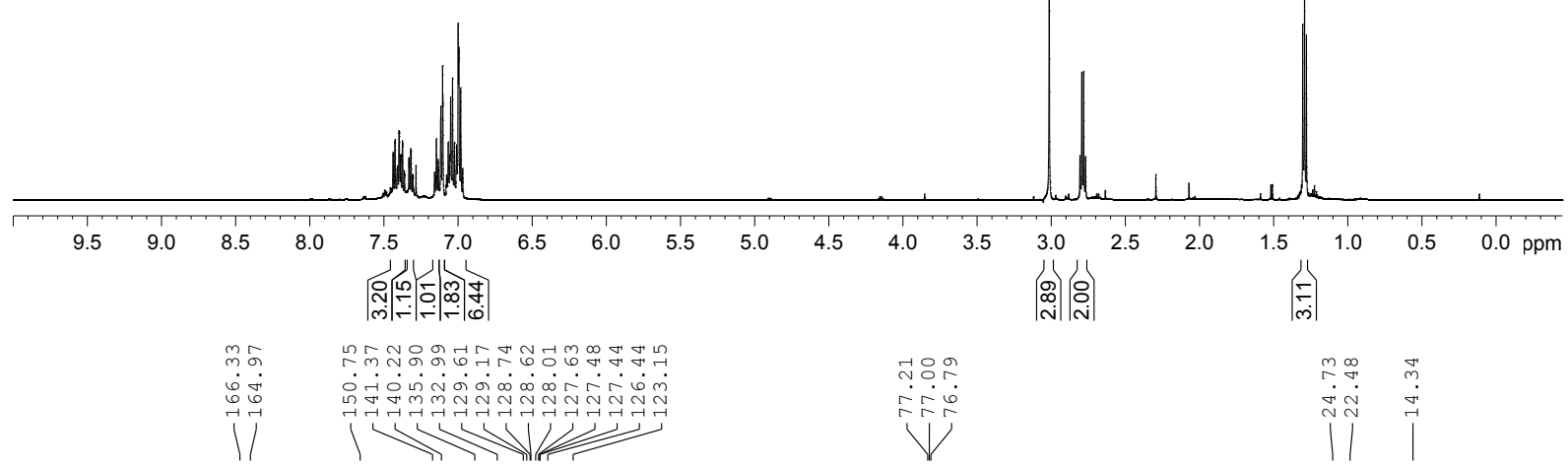
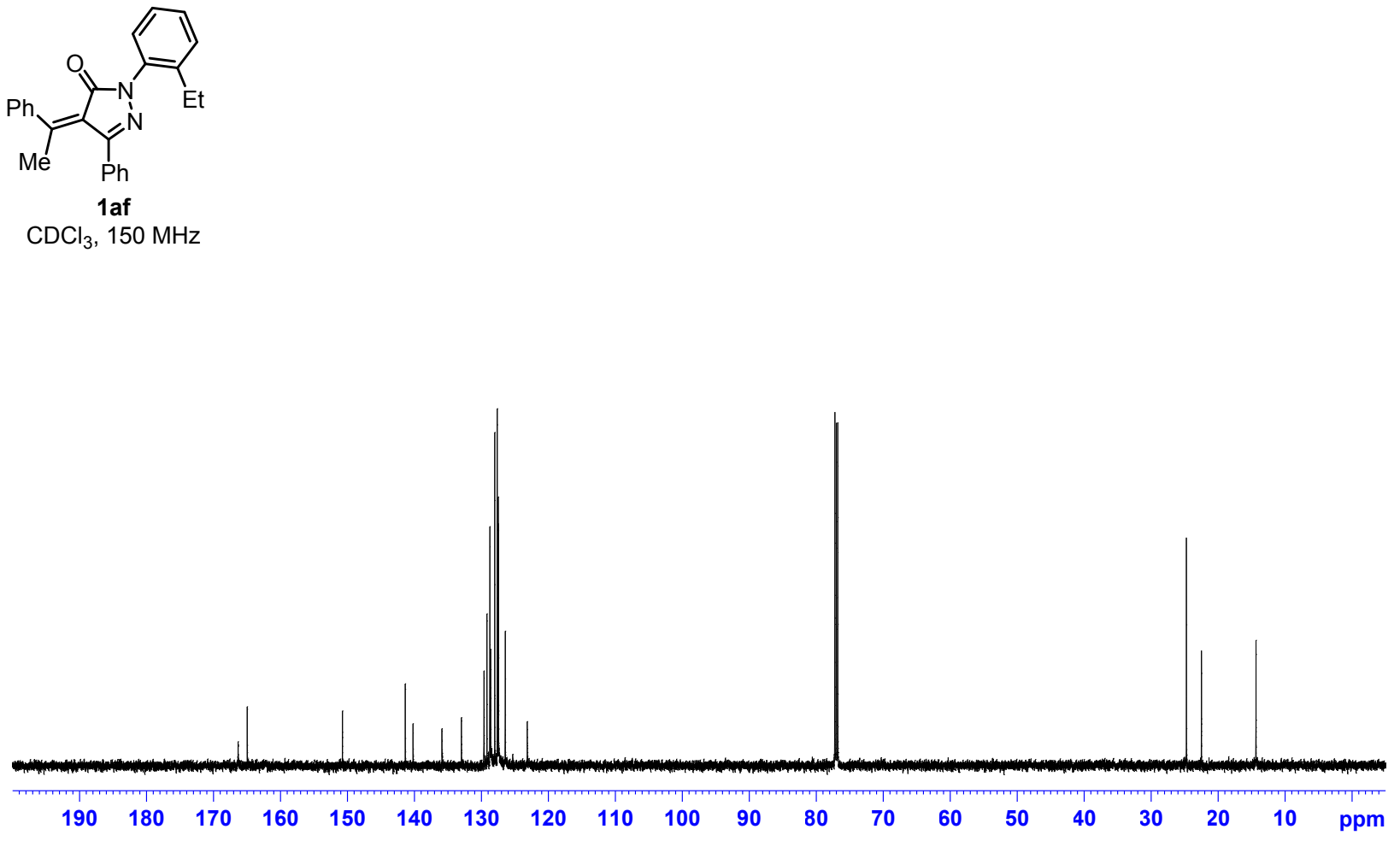

S13 


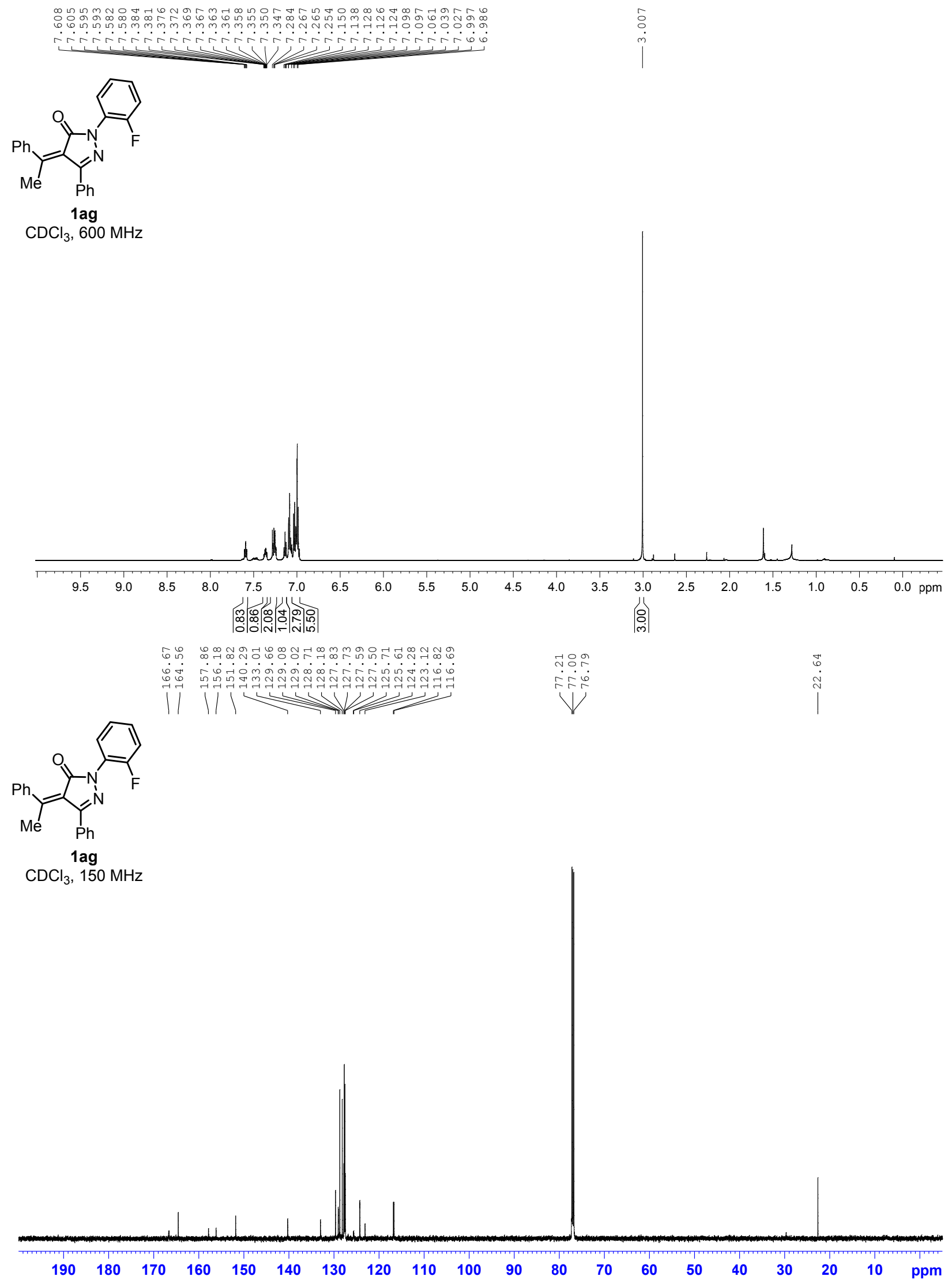



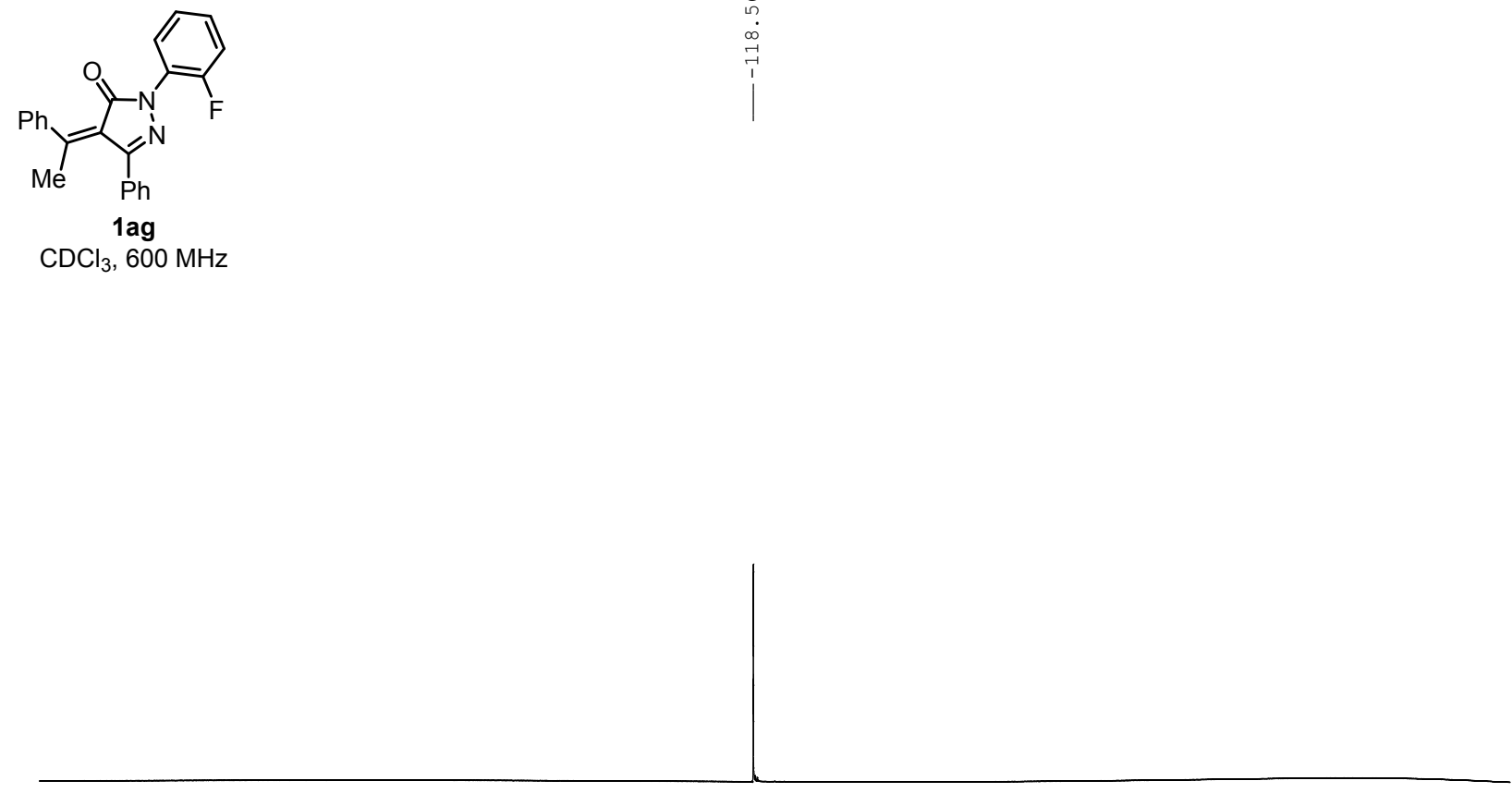

\begin{tabular}{|c|c|c|c|c|c|c|c|c|}
\hline-80 & -90 & -100 & -110 & -120 & -130 & -140 & -150 & -160 \\
\hline
\end{tabular}



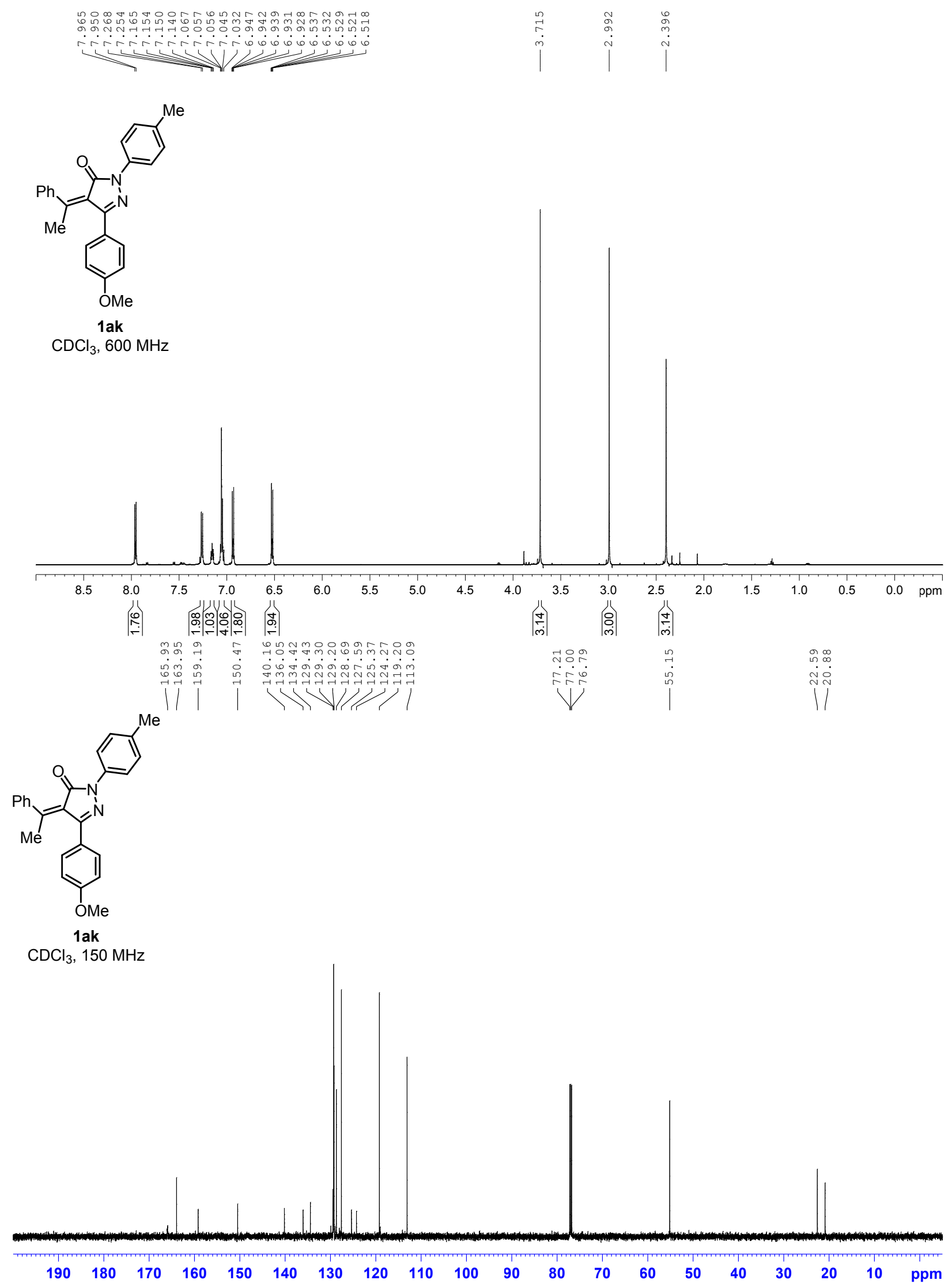


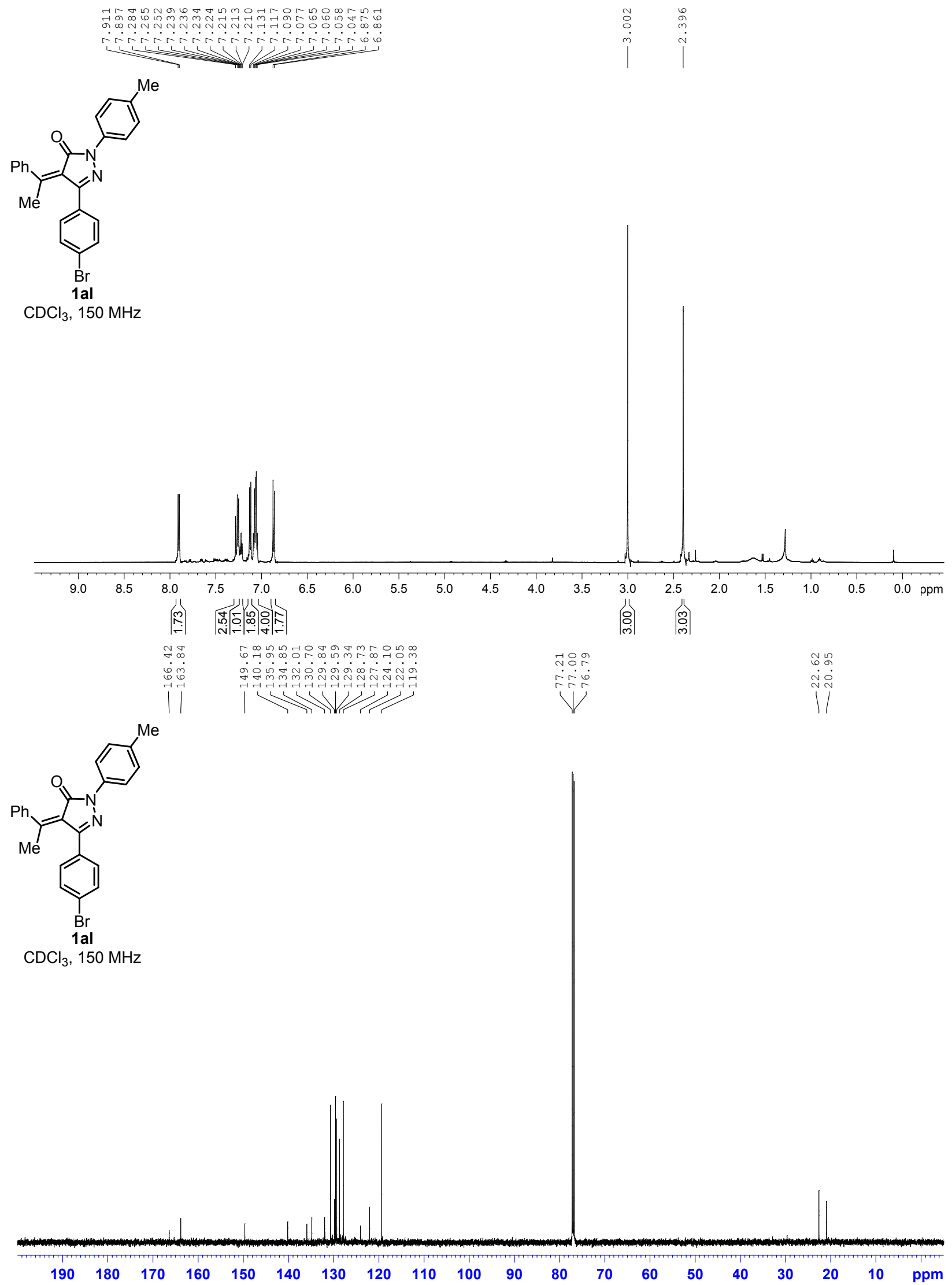



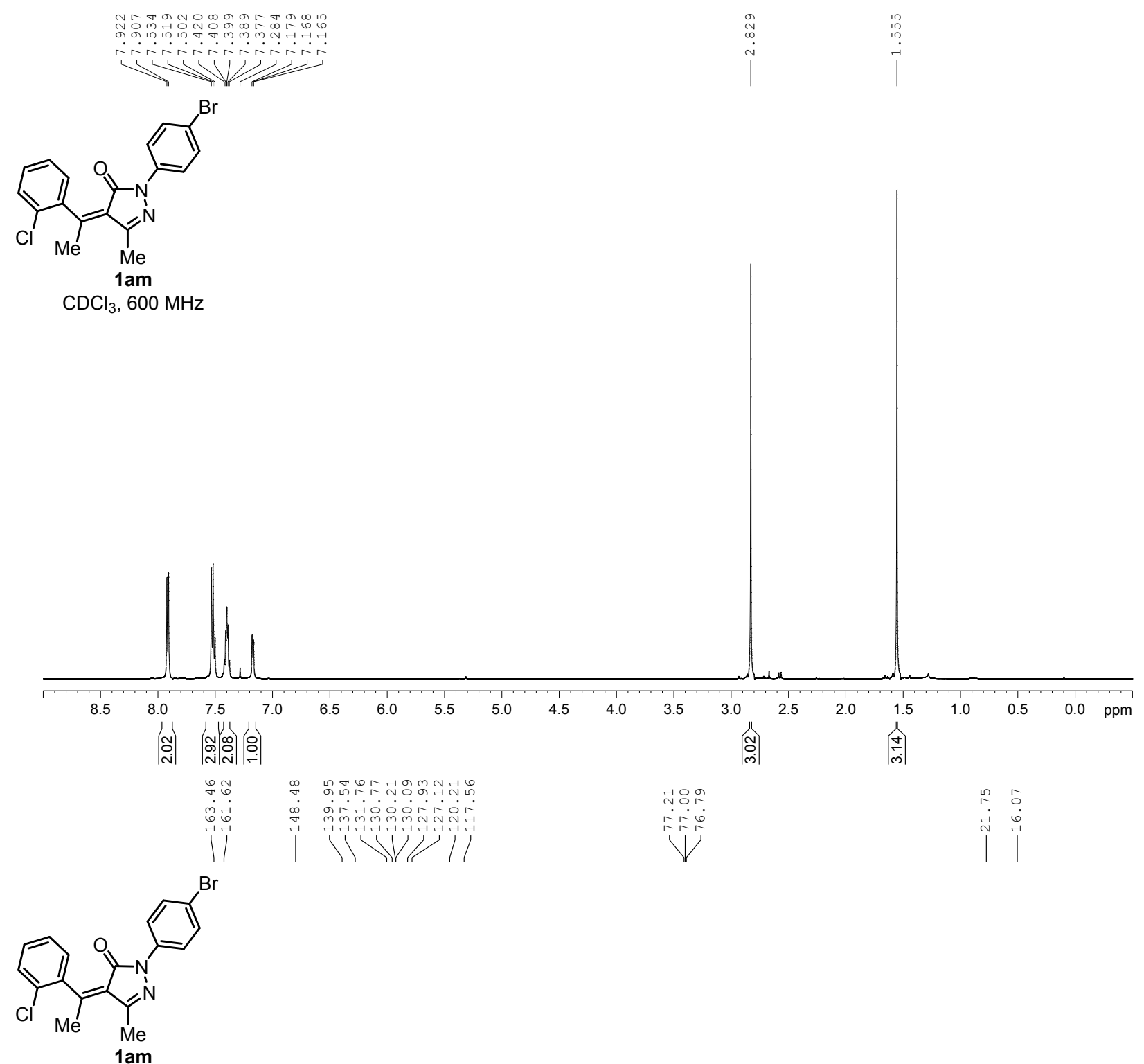

$\mathrm{CDCl}_{3}, 150 \mathrm{MHz}$

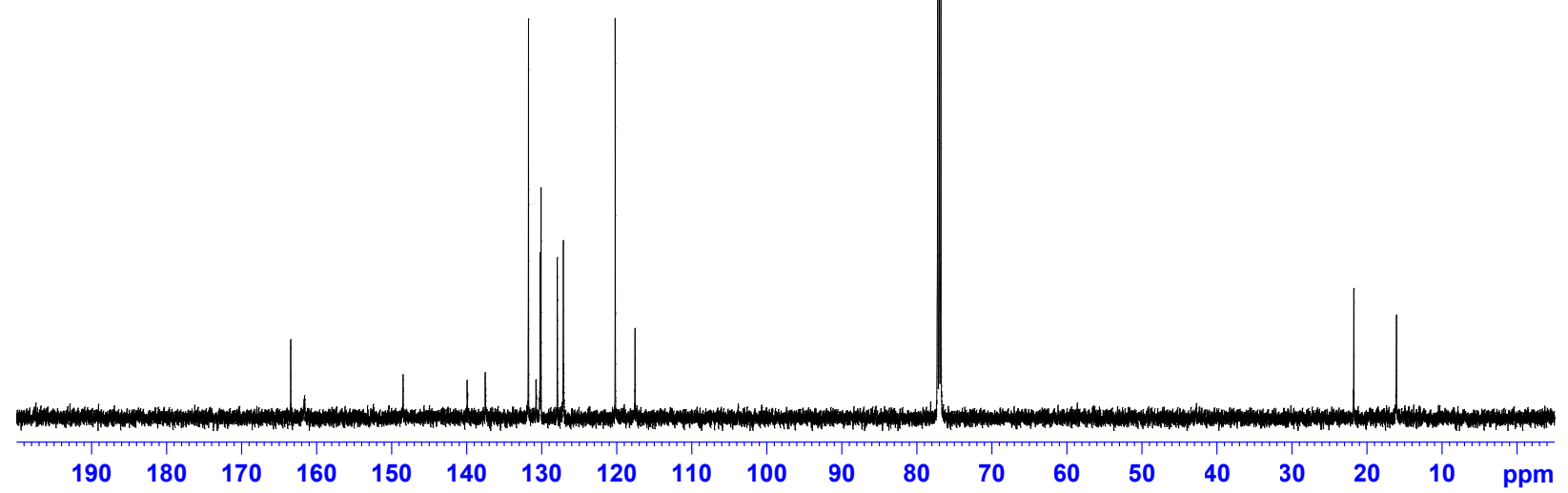




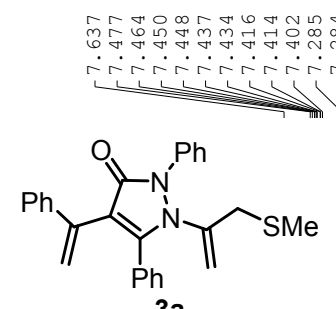

$\mathrm{CDCl}_{3}, 600 \mathrm{MHz}$
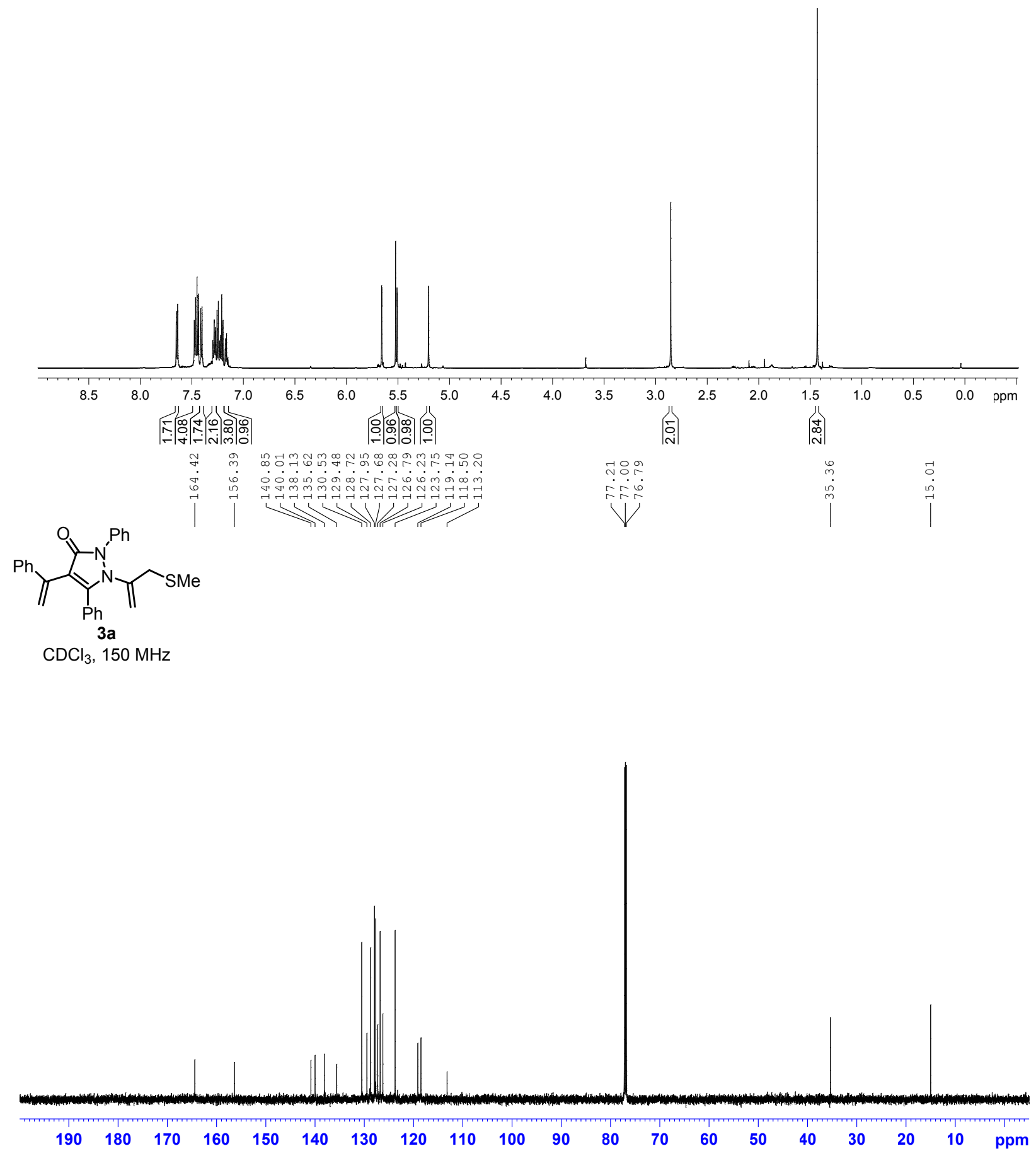

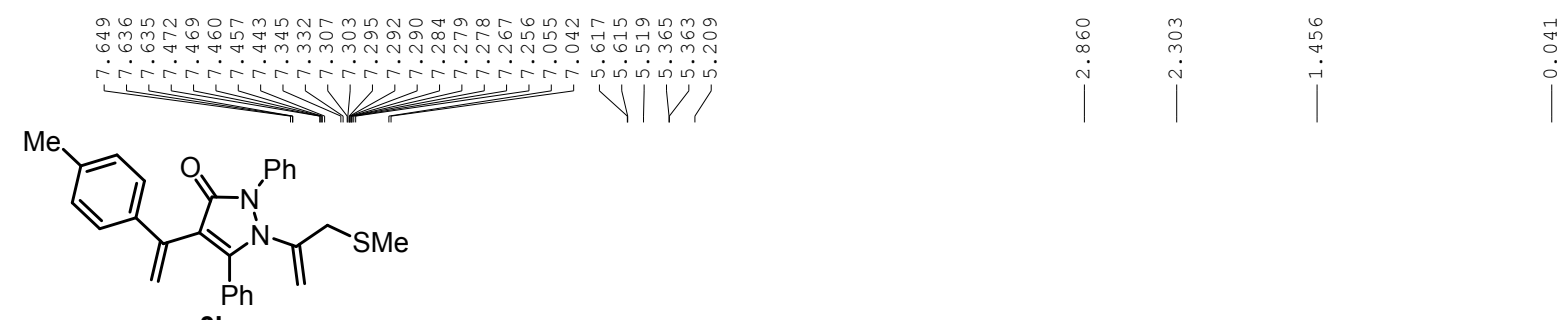

$3 b$

$\mathrm{CDCl}_{3}, 600 \mathrm{MHz}$

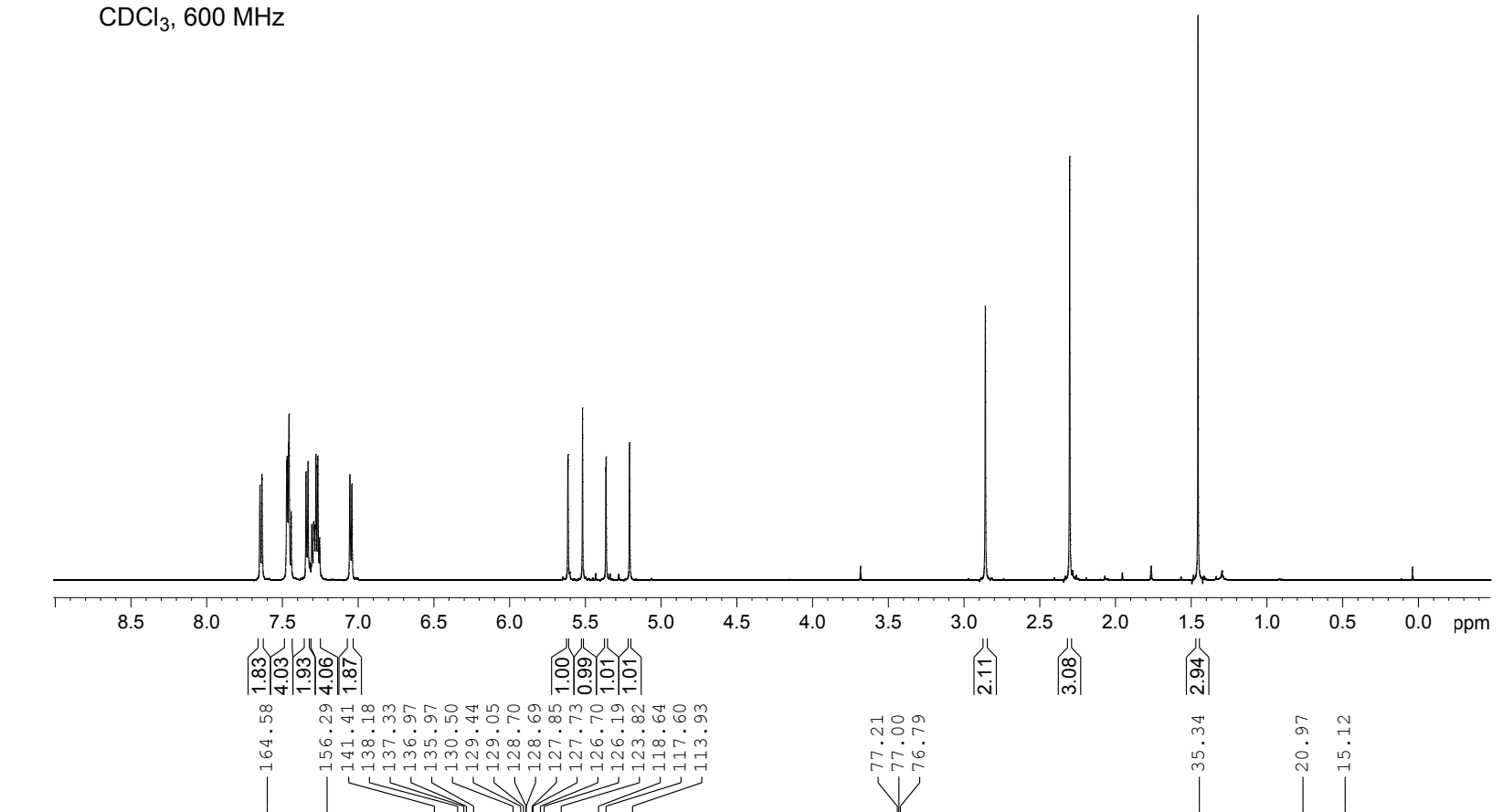<smiles>C=C(c1ccc(C)cc1)c1c(-c2ccccc2)n(C(=C)CC(C)C)n(-c2ccccc2)c1=O</smiles>

3b

$\mathrm{CDCl}_{3}, 150 \mathrm{MHz}$

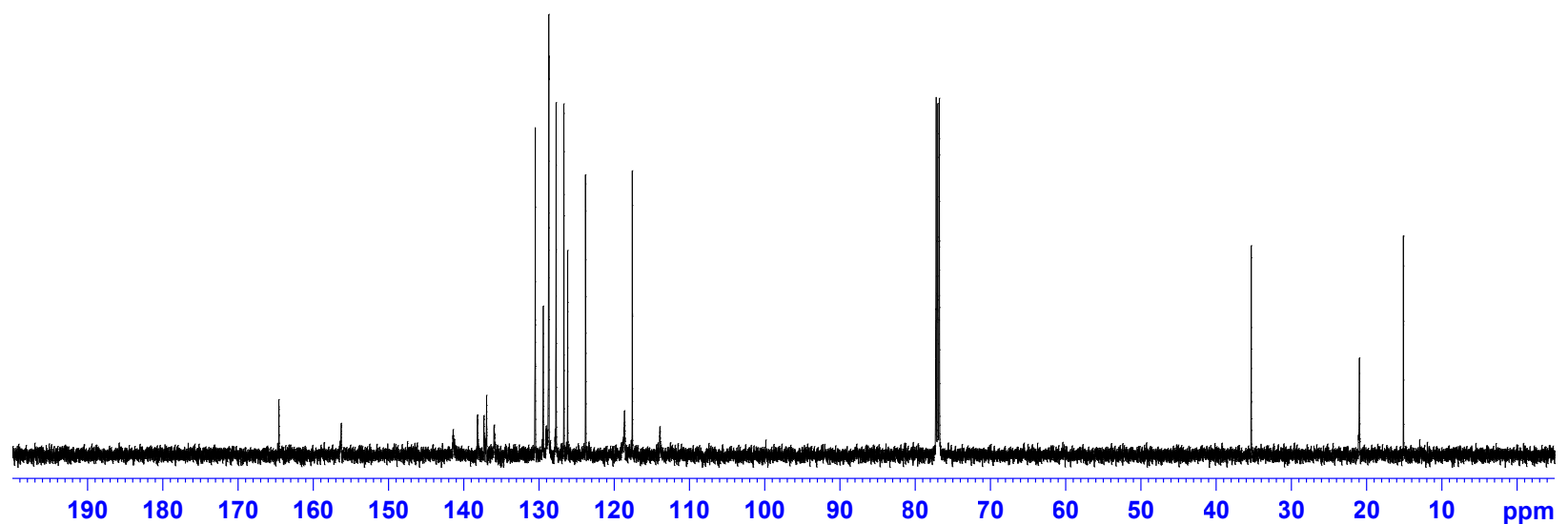



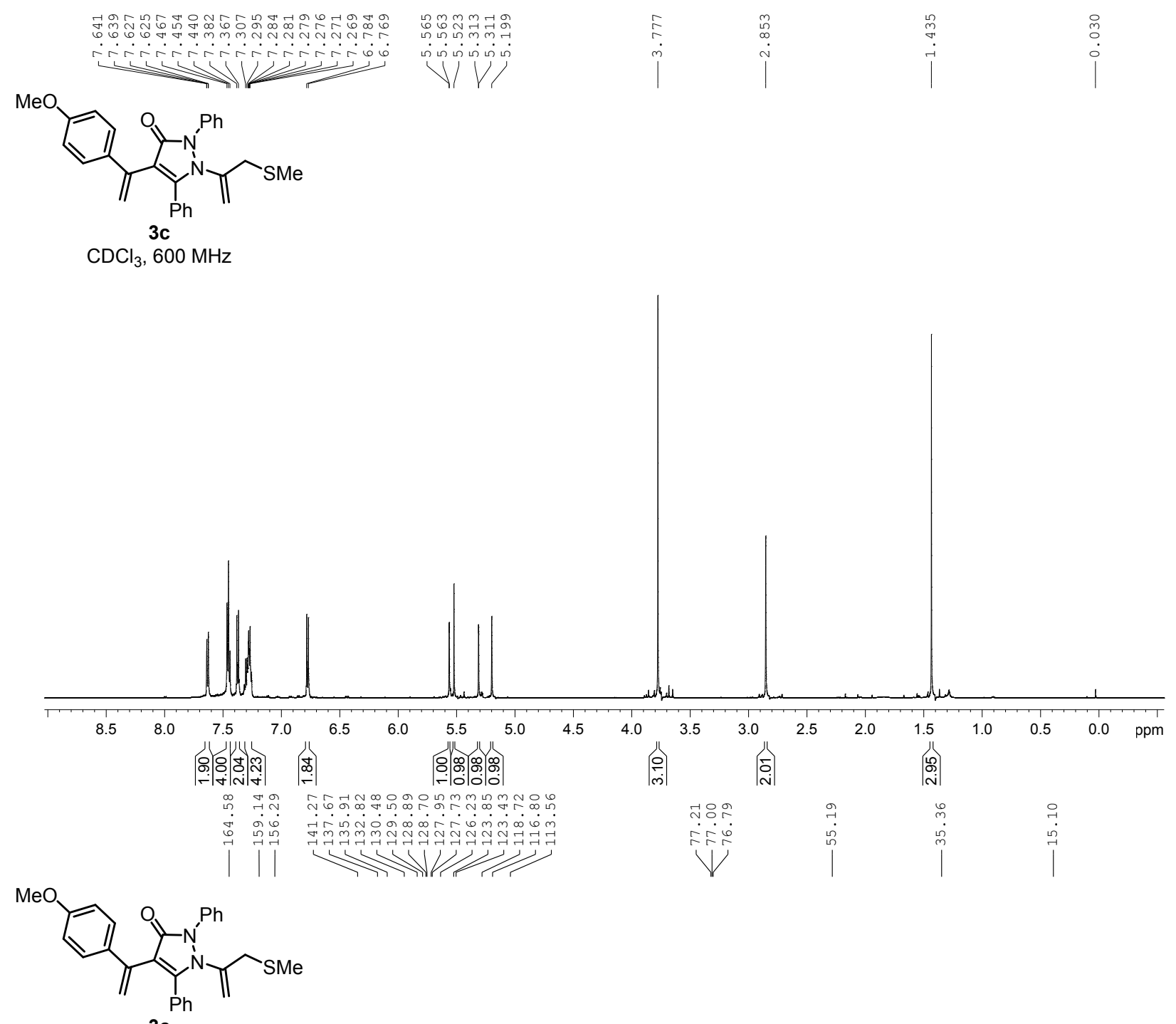

$\mathrm{CDCl}_{3}, 150 \mathrm{MHz}$

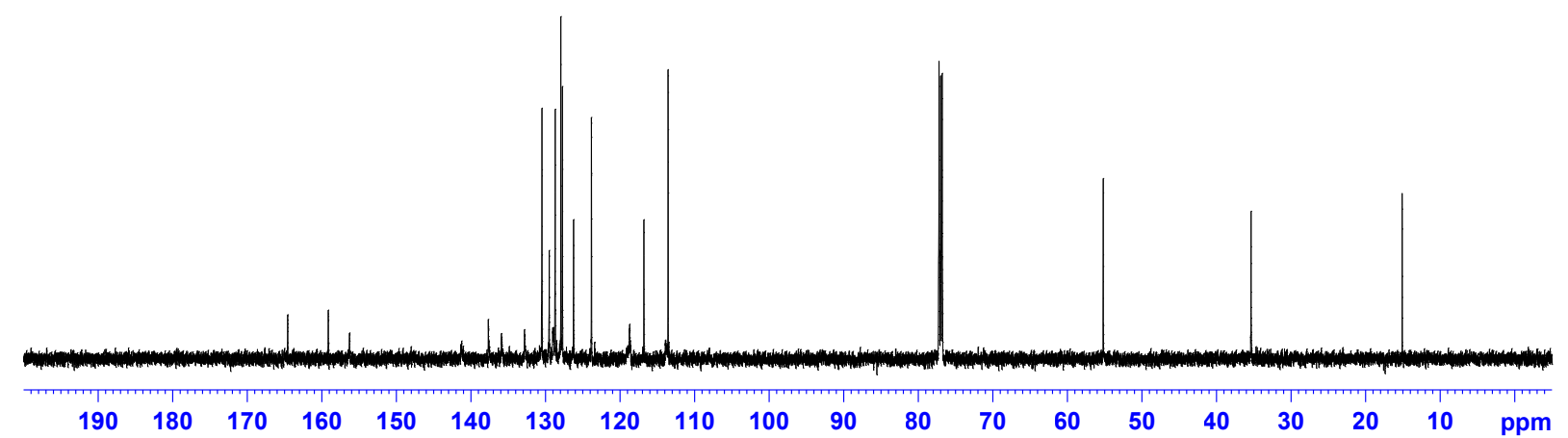



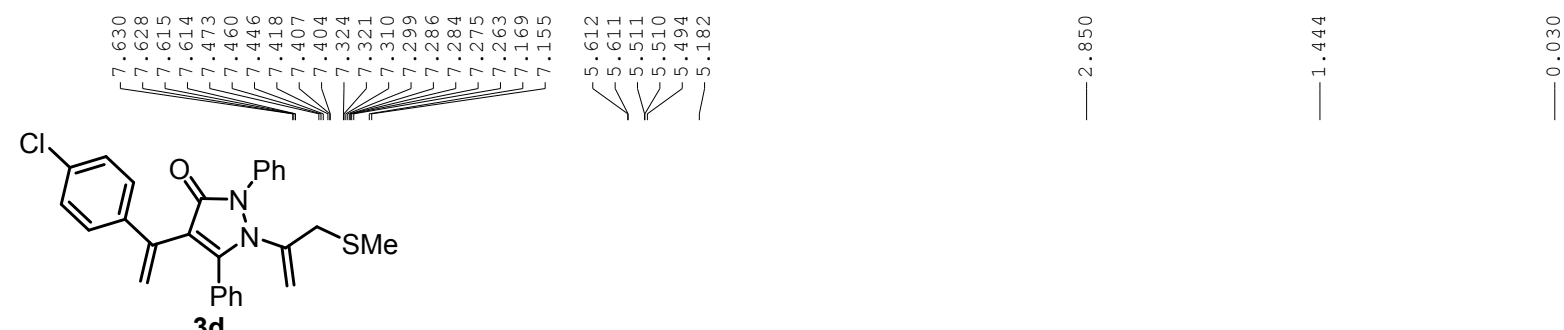

$\mathrm{CDCl}_{3}, 600 \mathrm{MHz}$
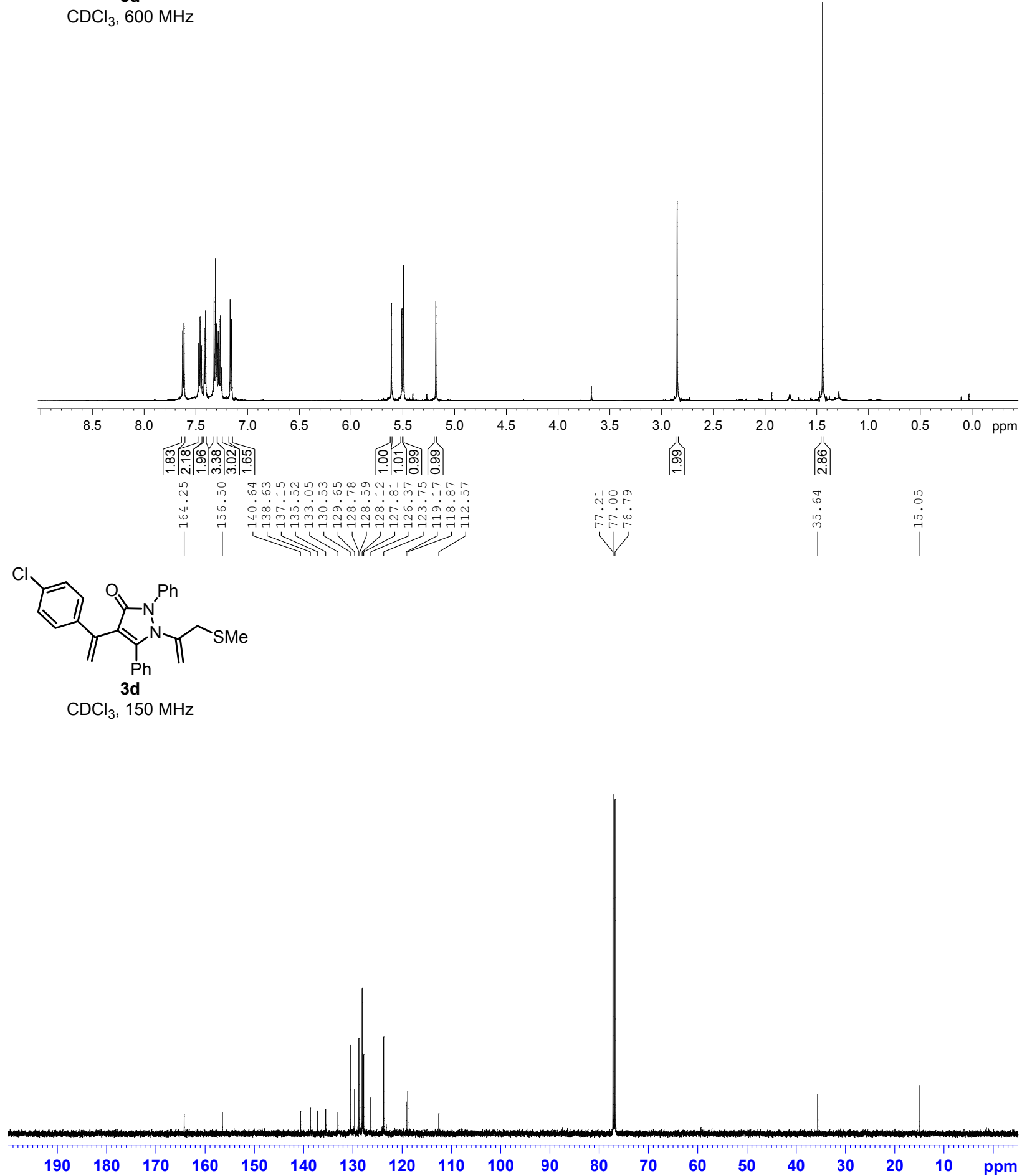


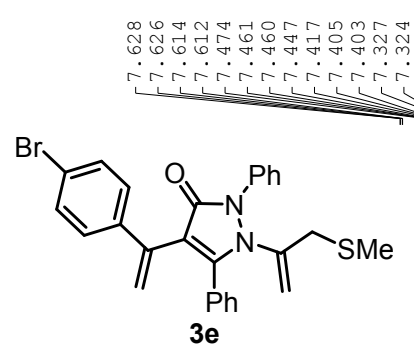

$\mathrm{CDCl}_{3}, 600 \mathrm{MHz}$
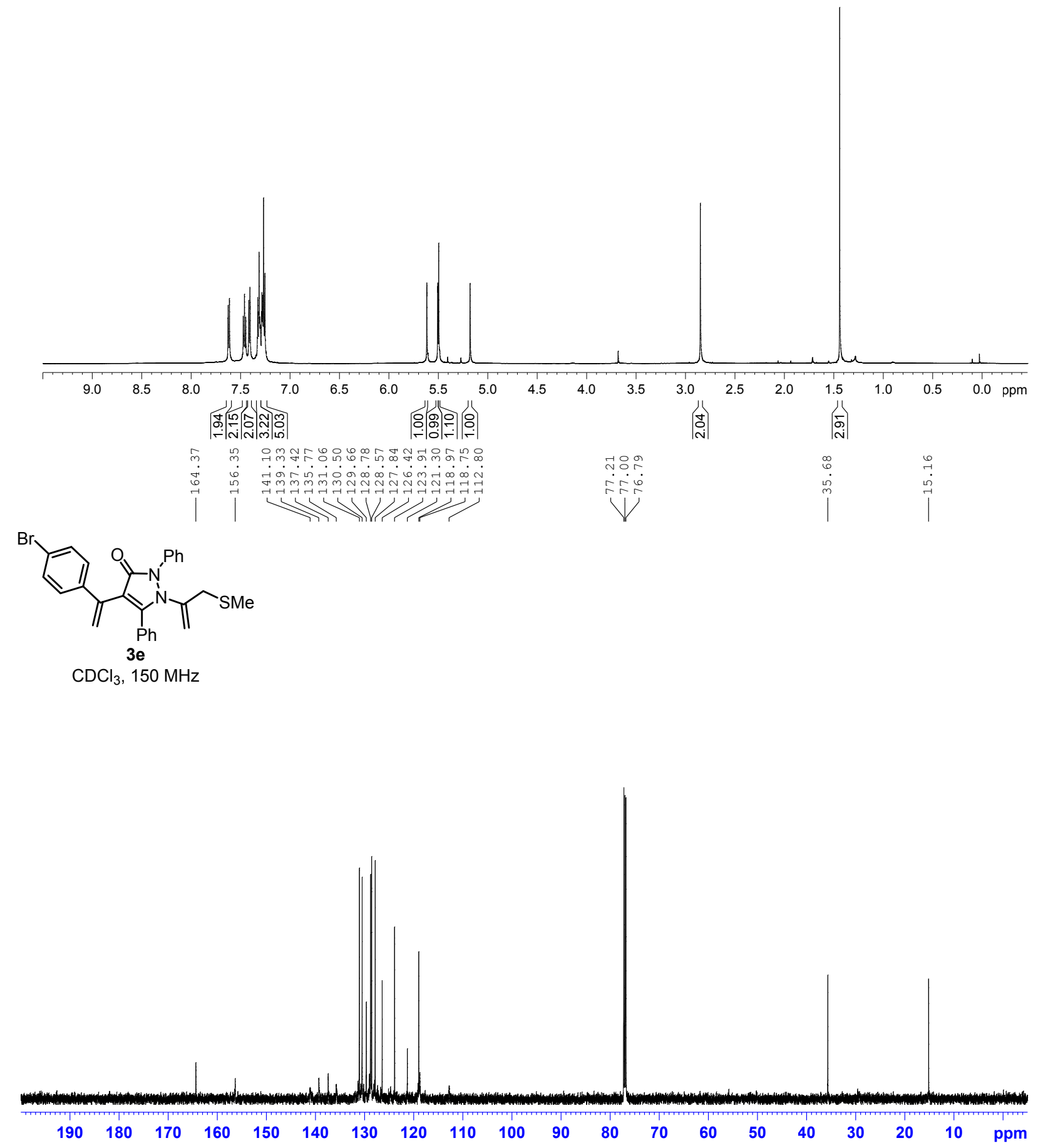

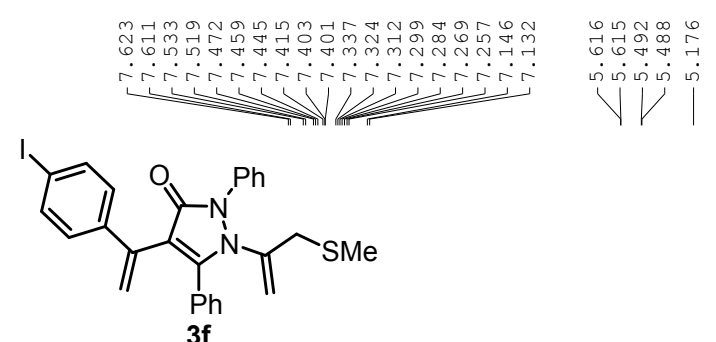

$\mathrm{CDCl}_{3}, 600 \mathrm{MHz}$
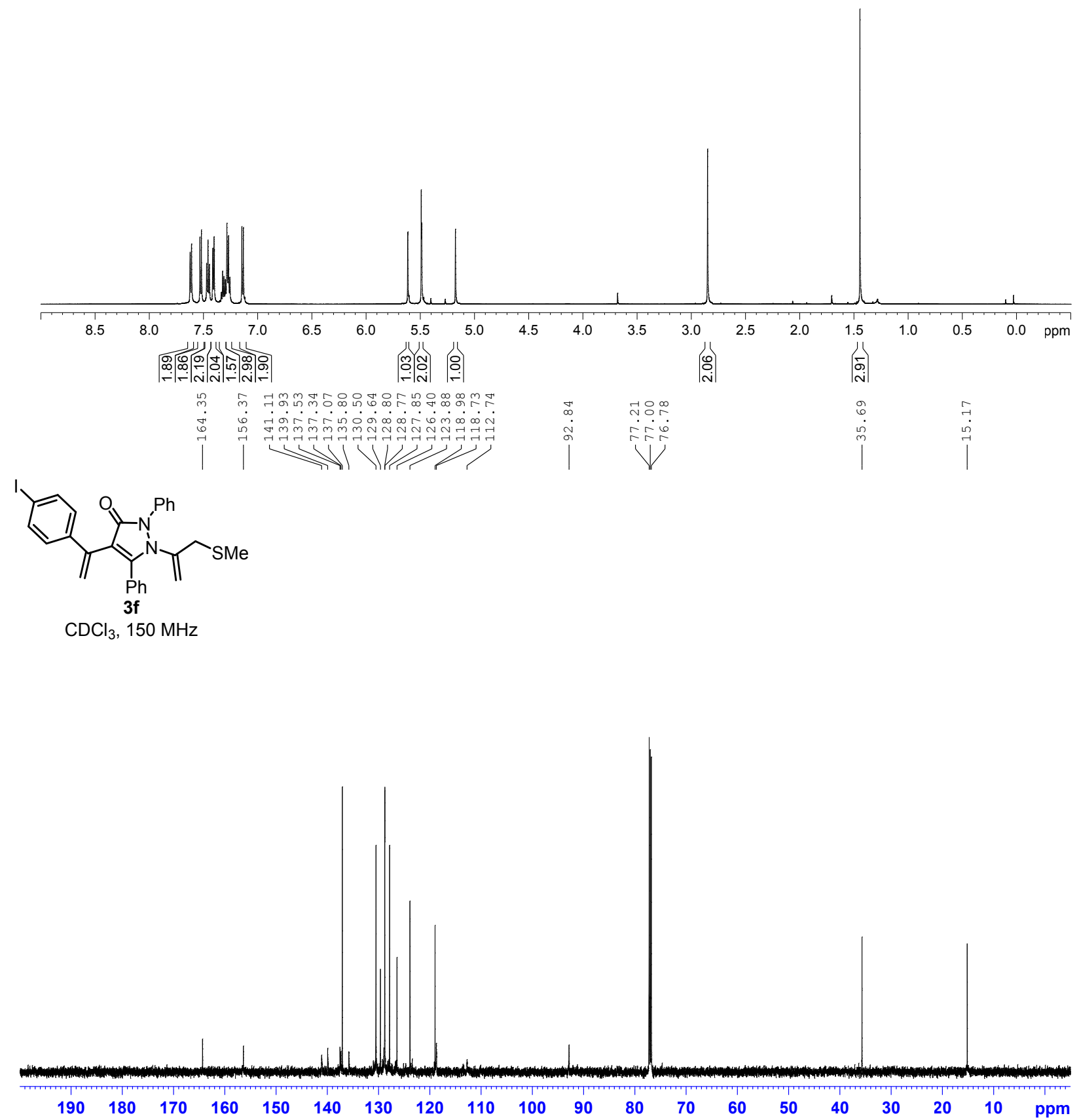

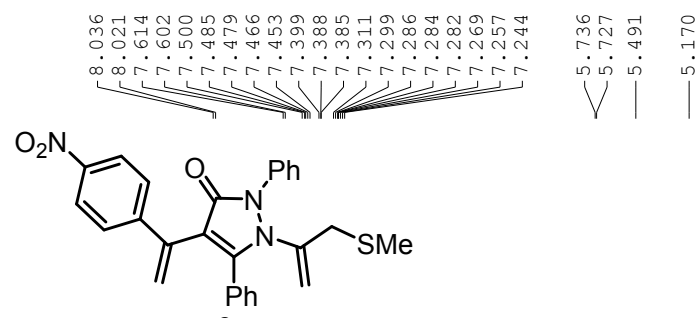

3g

$\mathrm{CDCl}_{3}, 600 \mathrm{MHz}$
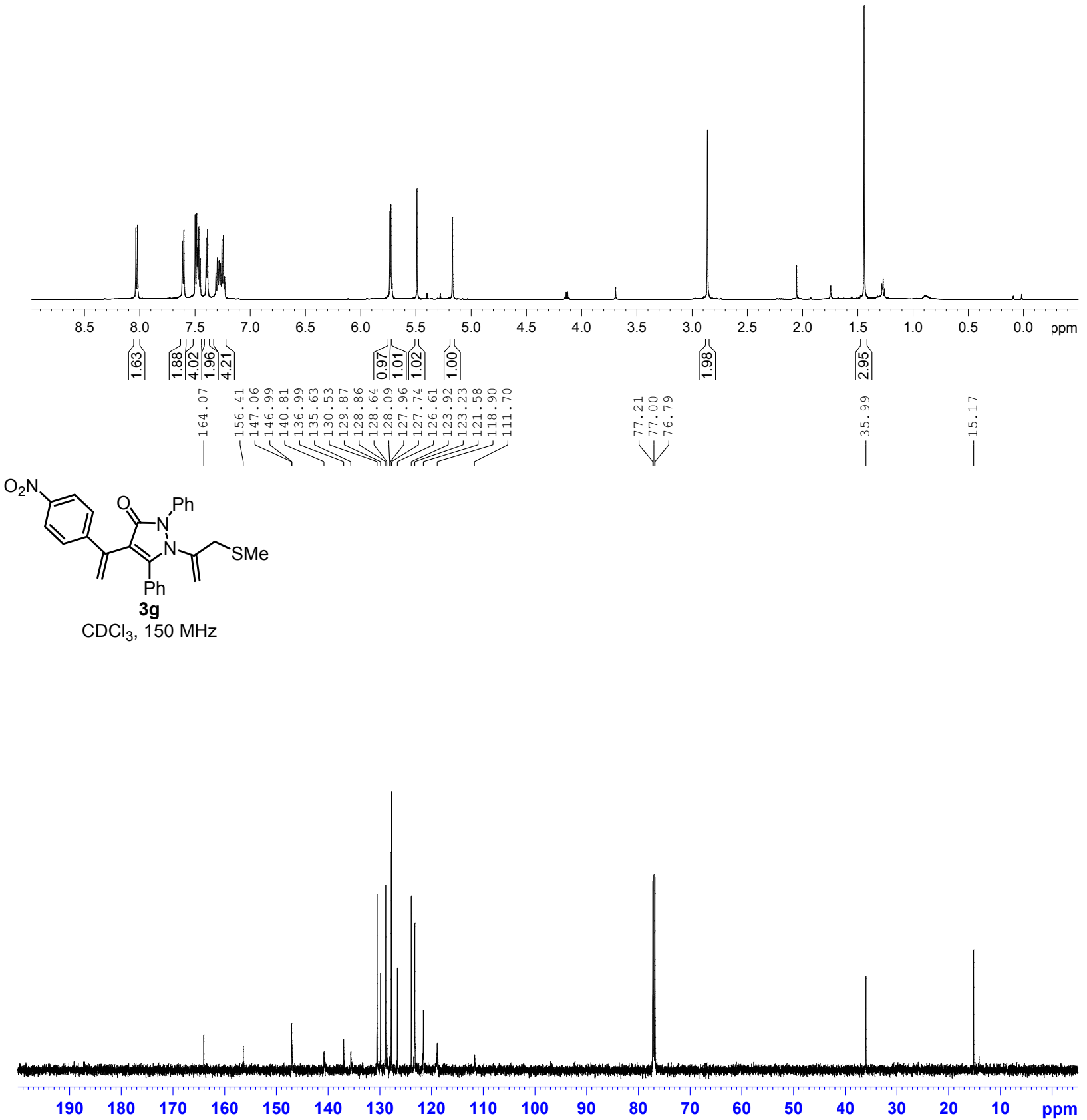

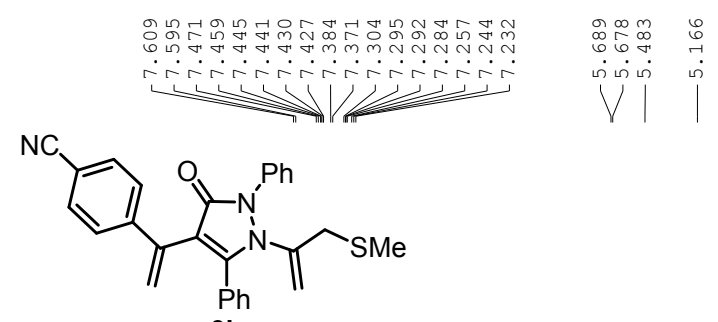

3h

$\mathrm{CDCl}_{3}, 600 \mathrm{MHz}$
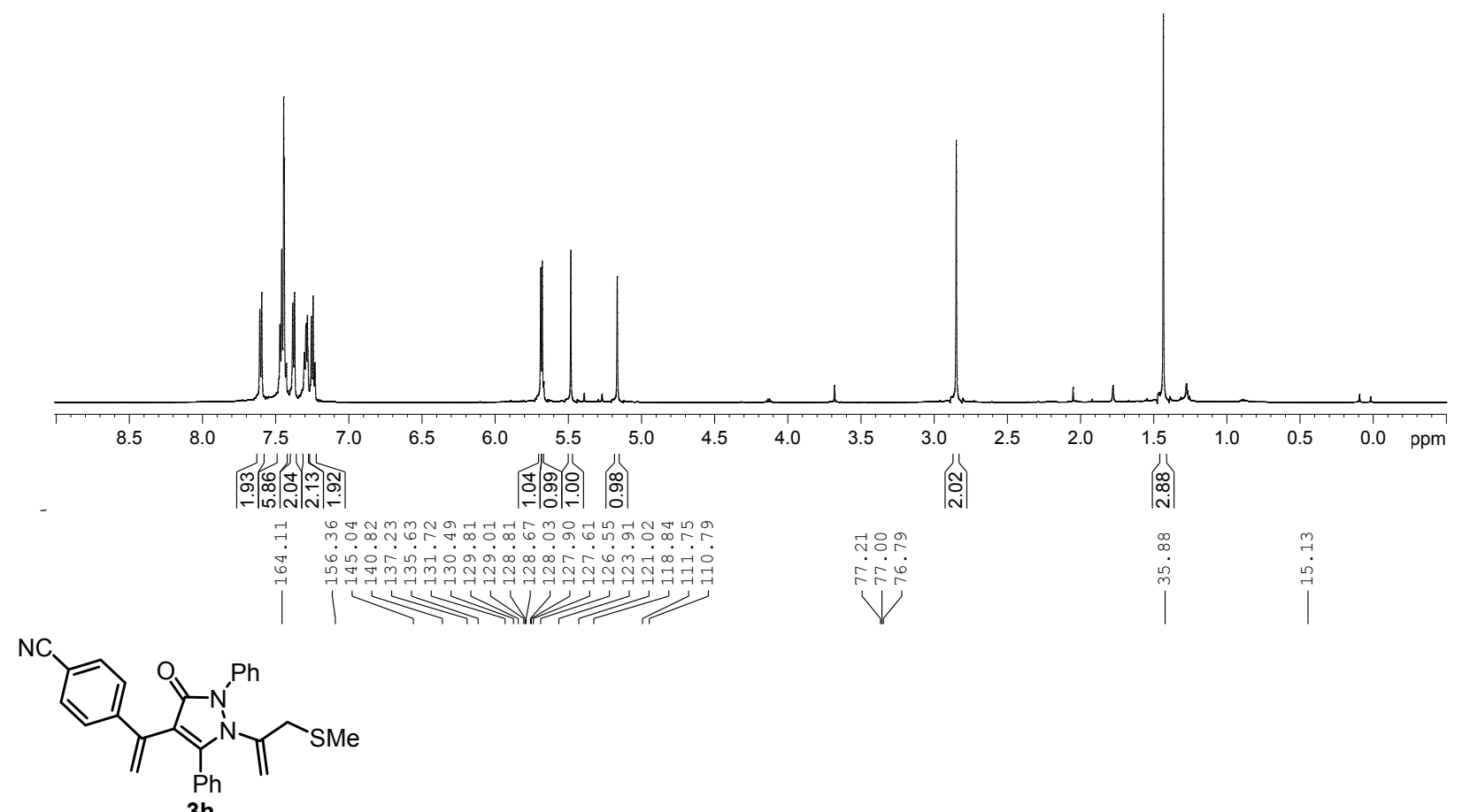

$\mathrm{CDCl}_{3}, 150 \mathrm{MHz}$

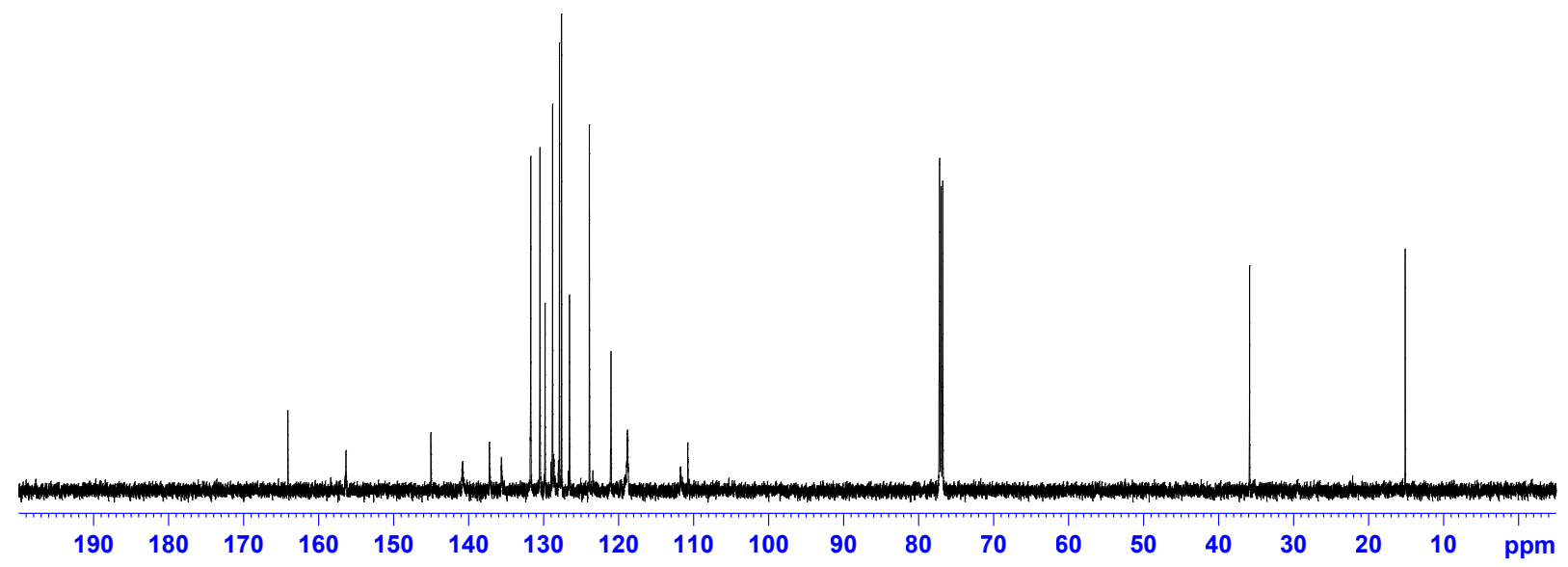




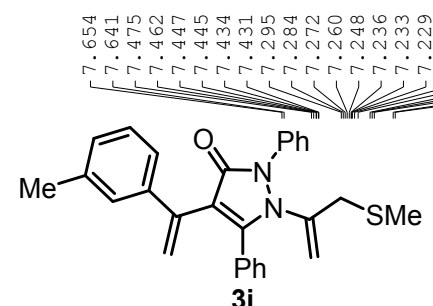

$\mathrm{CDCl}_{3}, 600 \mathrm{MHz}$
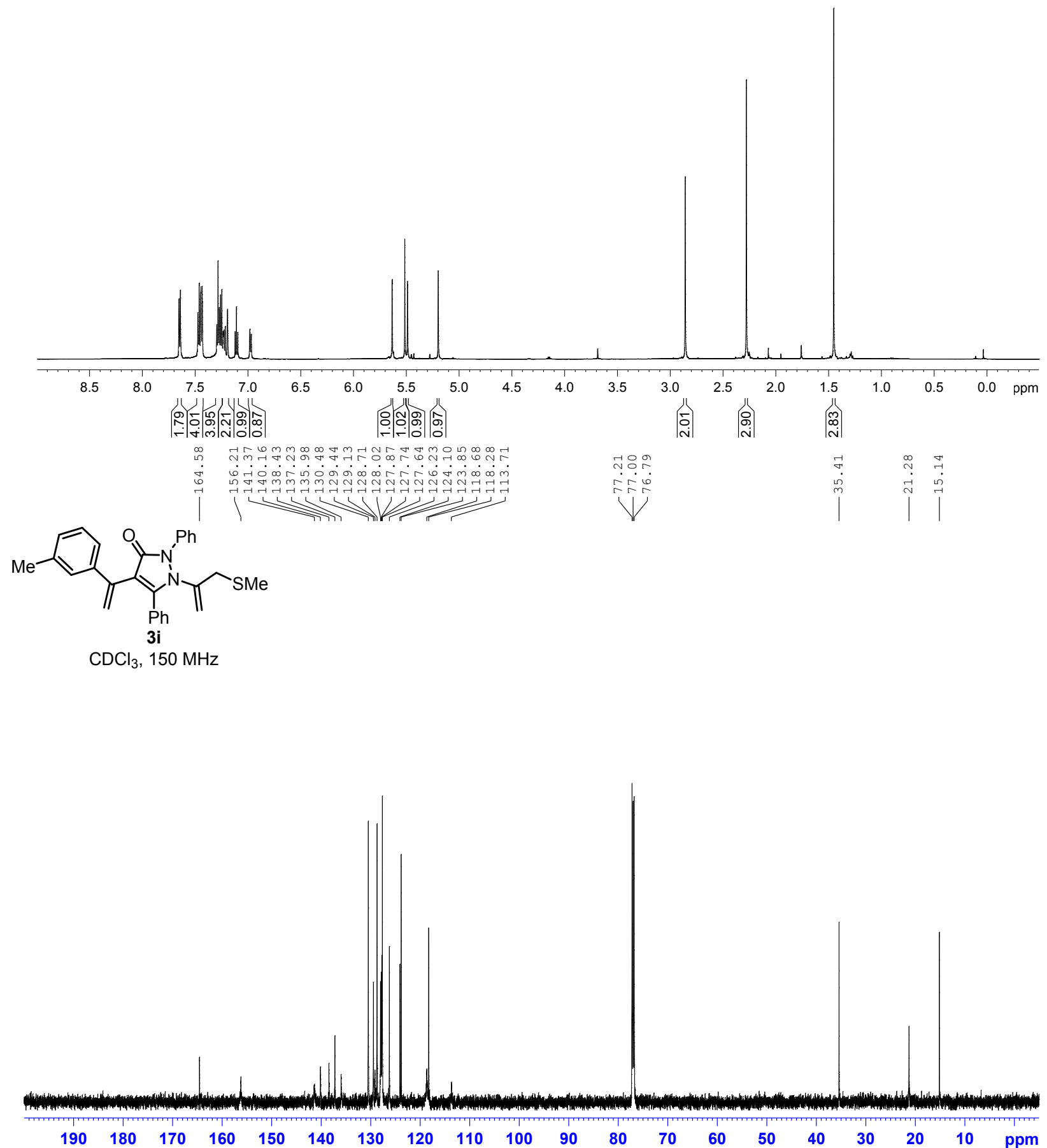


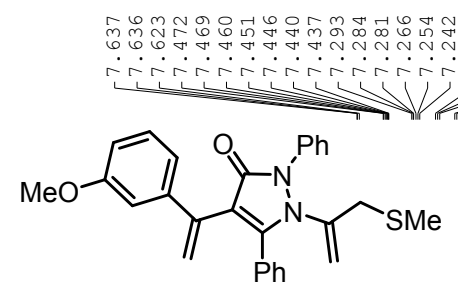

3j

$\mathrm{CDCl}_{3}, 600 \mathrm{MHz}$

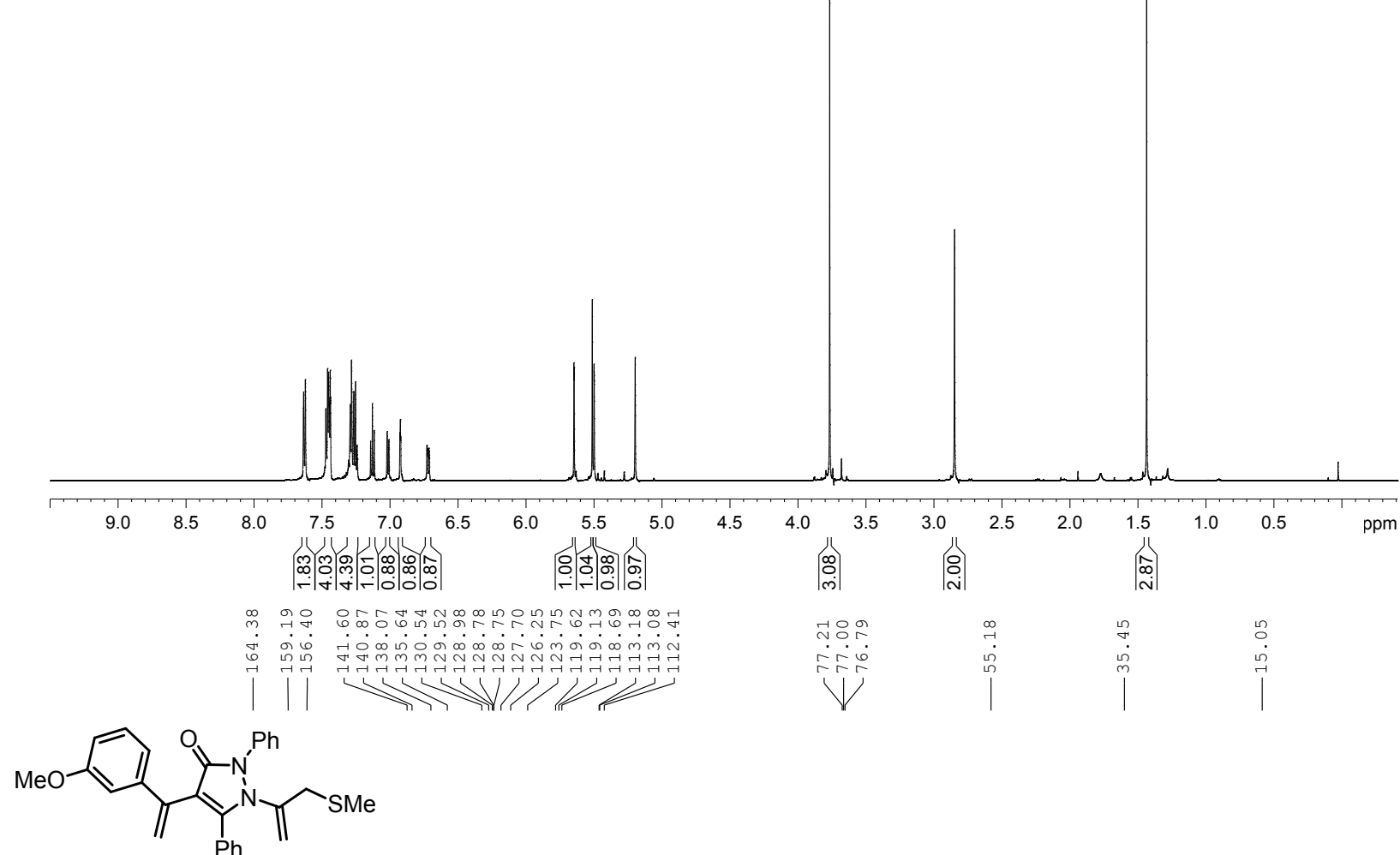

3j

$\mathrm{CDCl}_{3}, 150 \mathrm{MHz}$

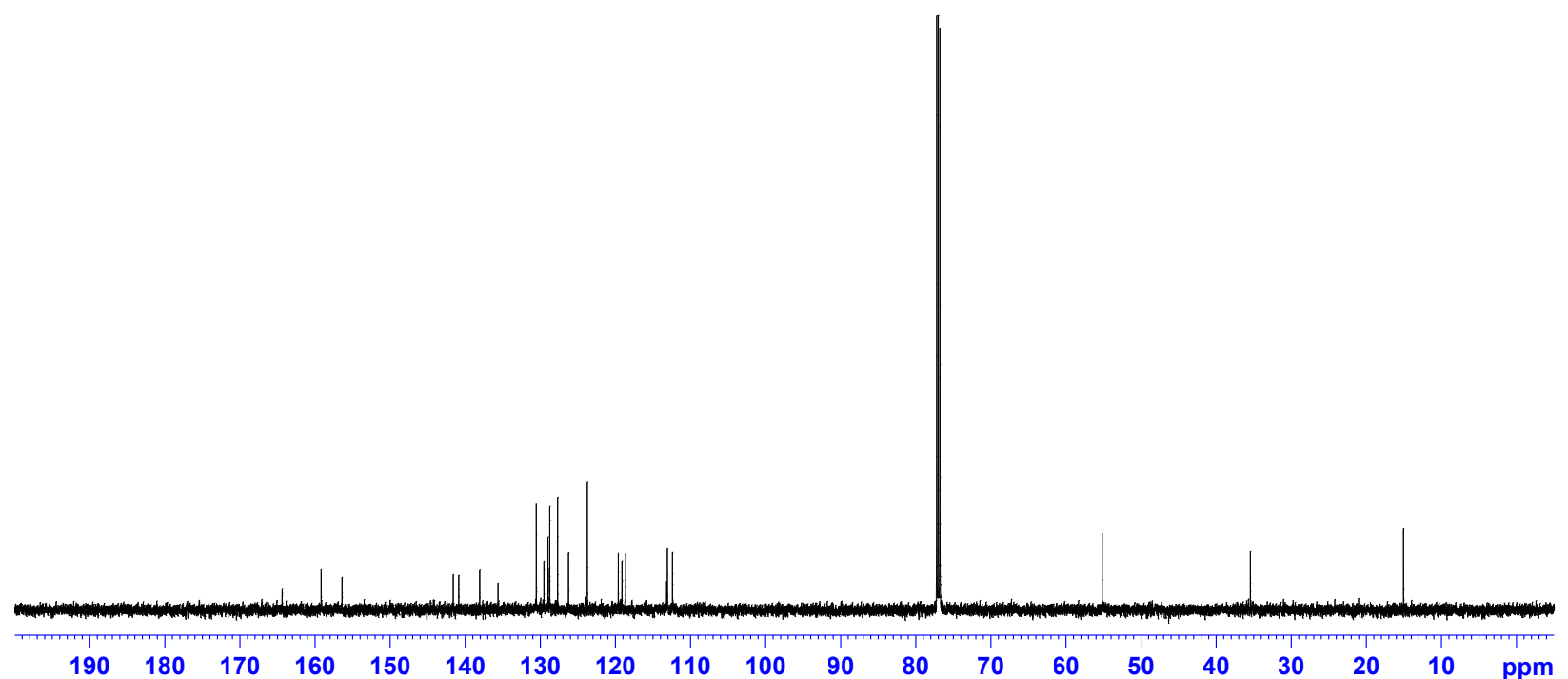




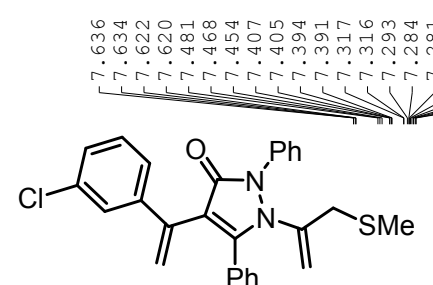

3k

$\mathrm{CDCl}_{3}, 600 \mathrm{MHz}$
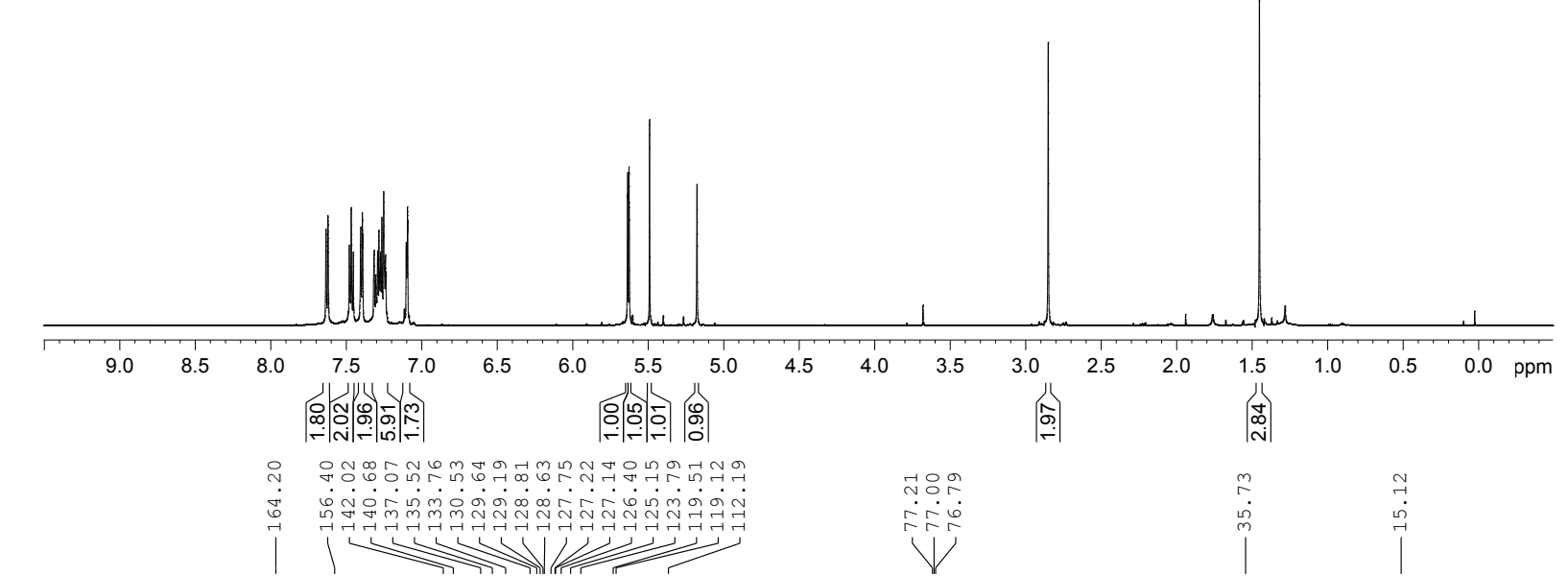

SMe

$3 k$

$\mathrm{CDCl}_{3}, 150 \mathrm{MHz}$

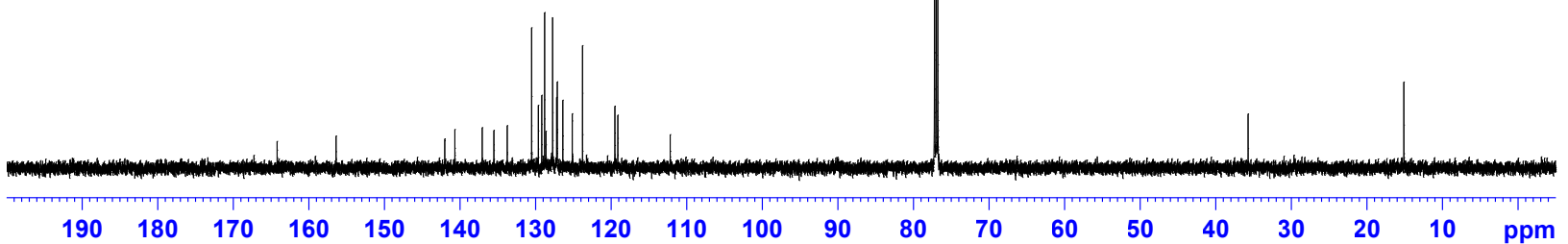




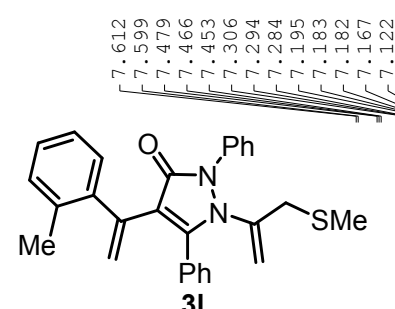

$\mathrm{CDCl}_{3}, 600 \mathrm{MHz}$
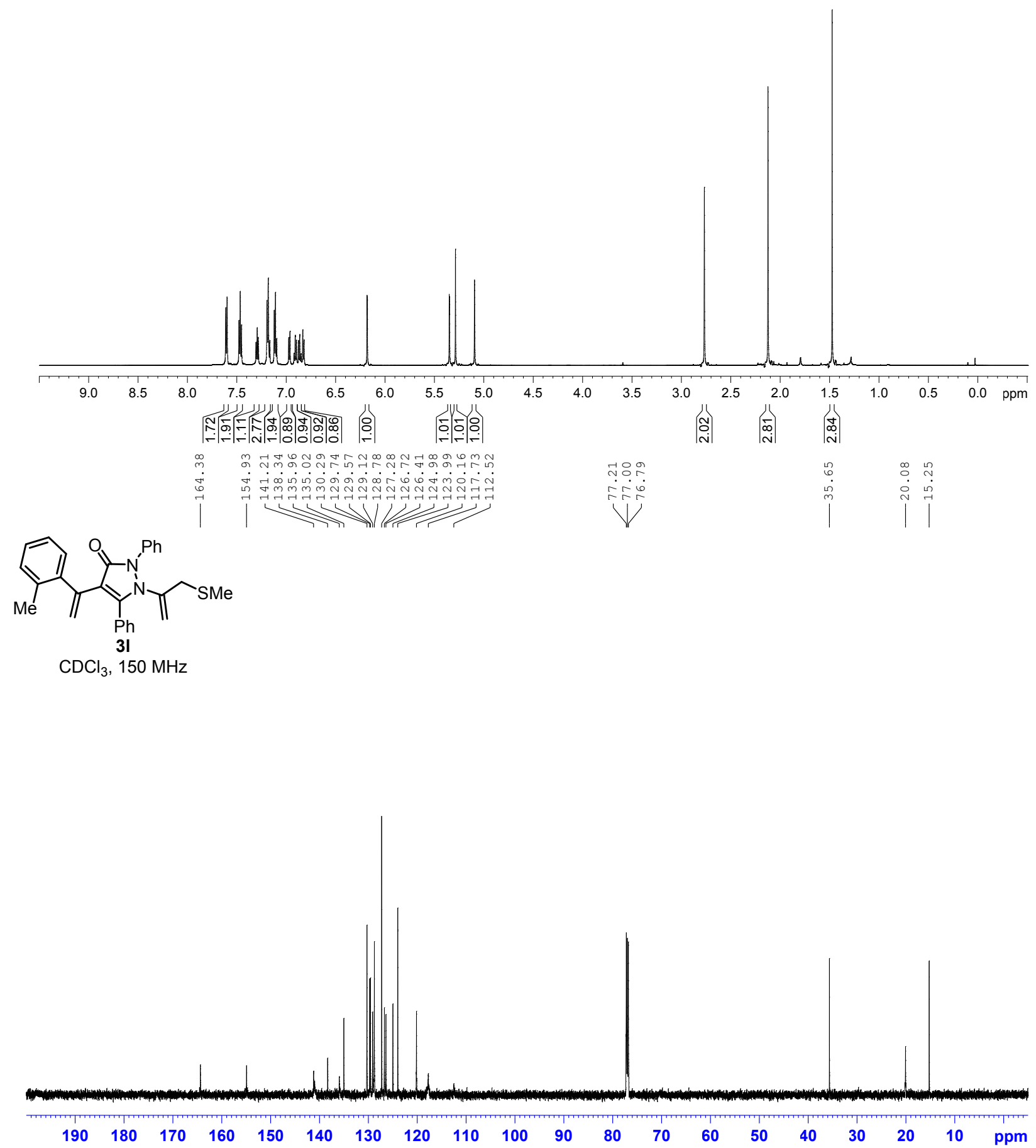


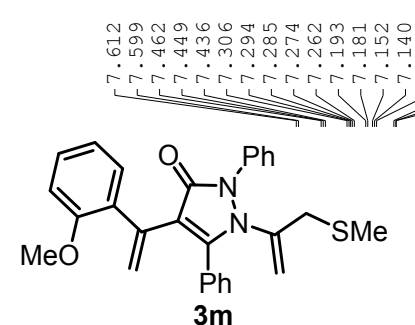

$\mathrm{CDCl}_{3}, 600 \mathrm{MHz}$
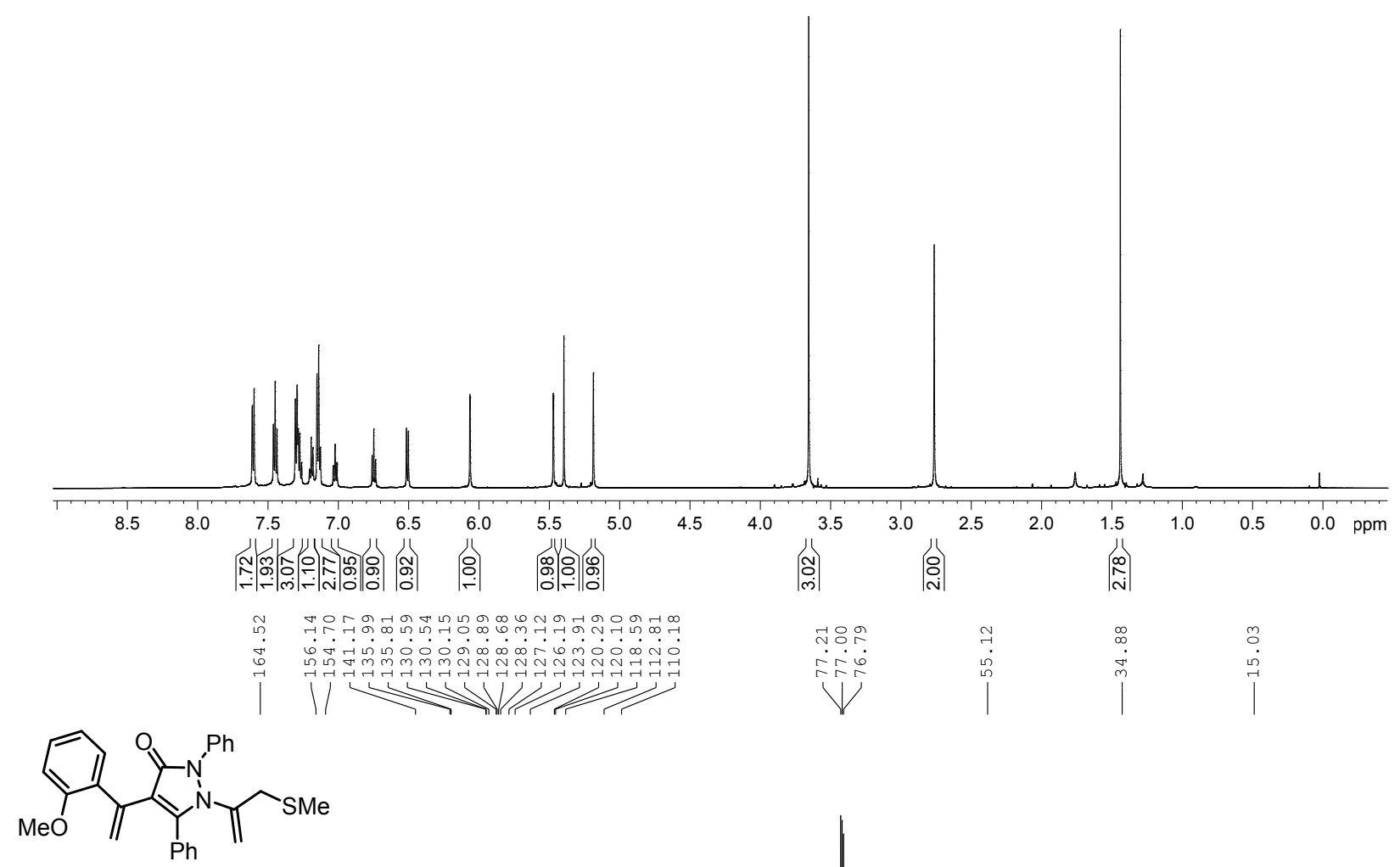

$\mathrm{CDCl}_{3}, 150 \mathrm{MHz}$

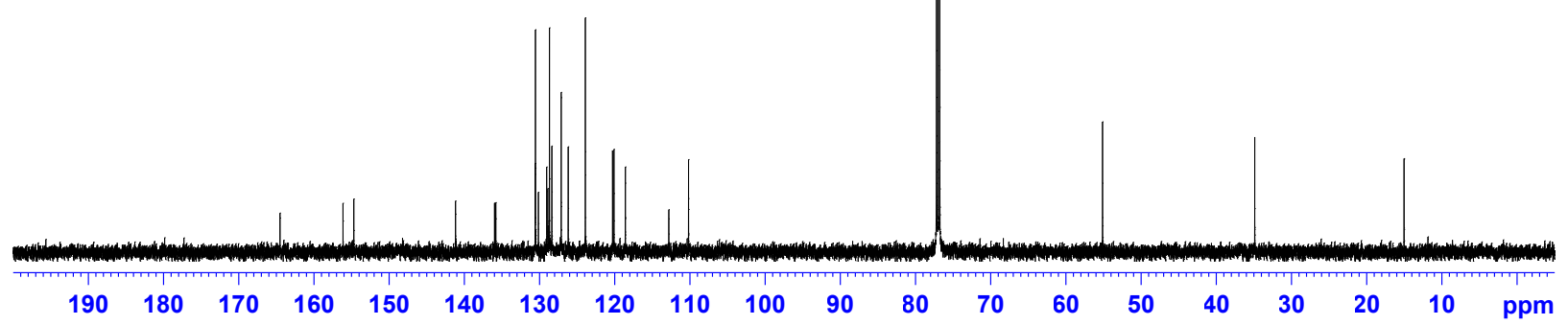




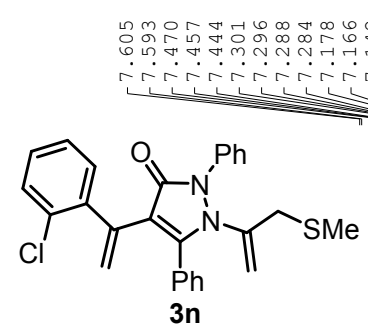

$\mathrm{CDCl}_{3}, 600 \mathrm{MHz}$
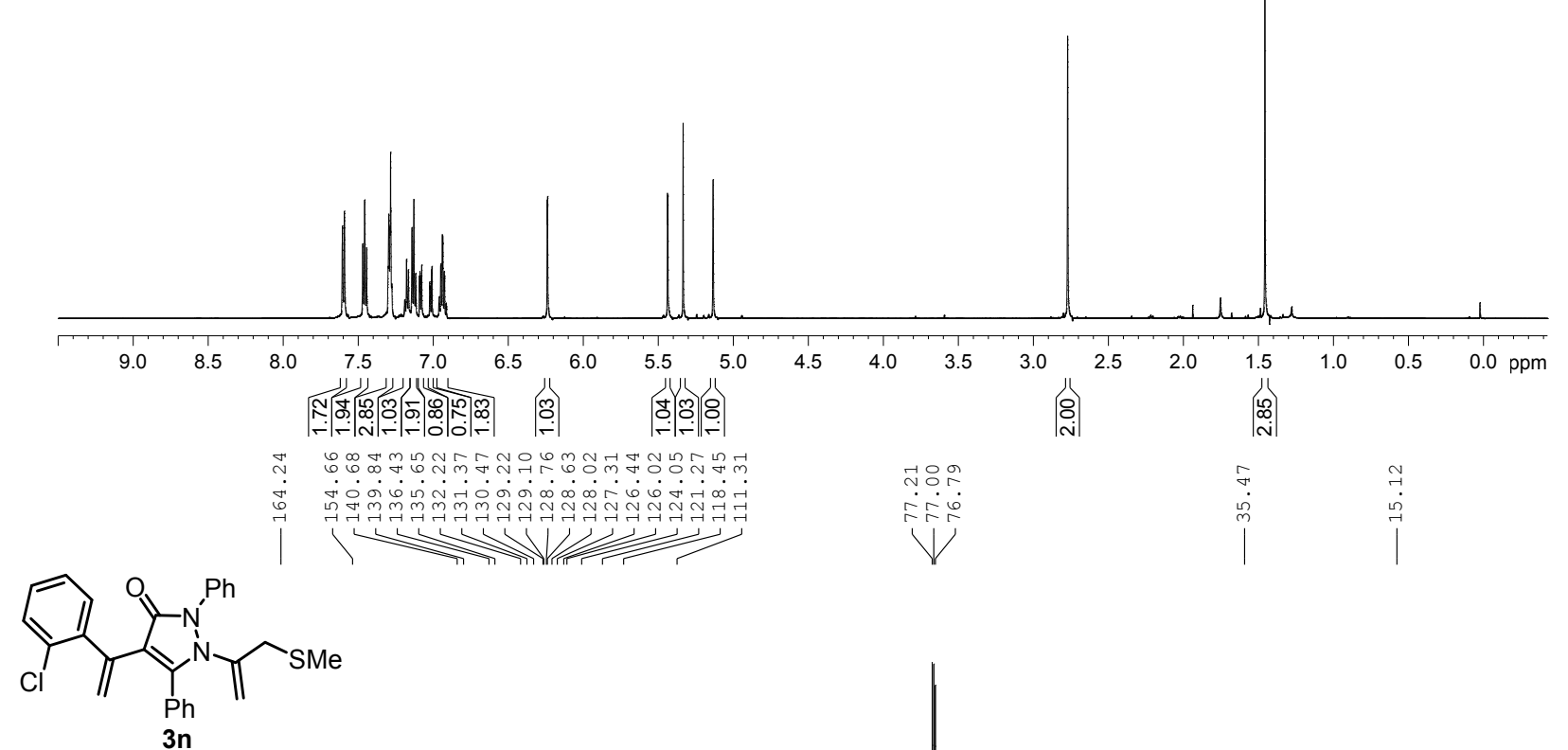

$\mathrm{CDCl}_{3}, 150 \mathrm{MHz}$

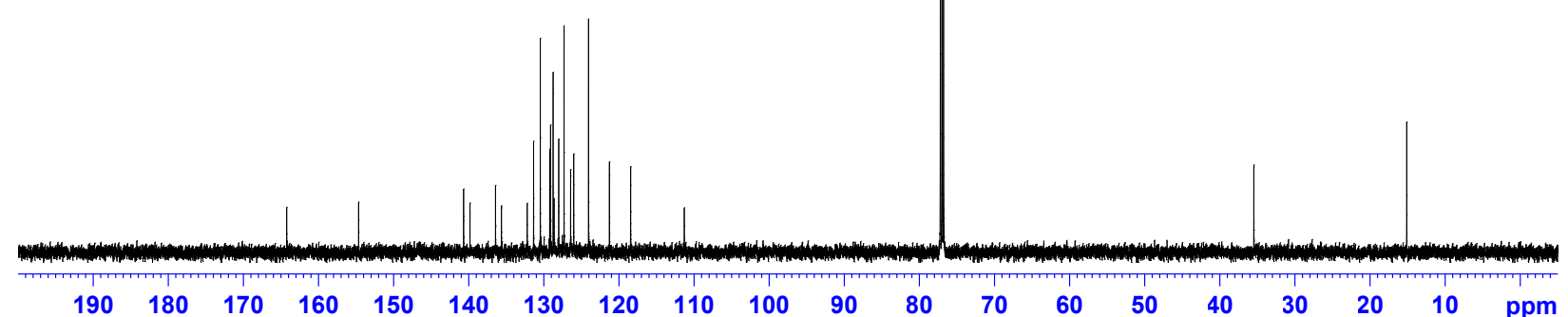




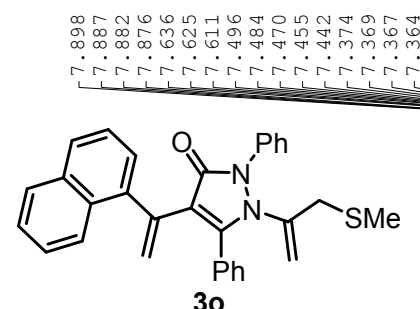

$\mathrm{CDCl}_{3}, 600 \mathrm{MHz}$



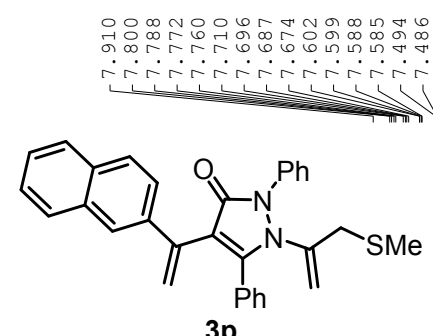

$\mathrm{CDCl}_{3}, 600 \mathrm{MHz}$
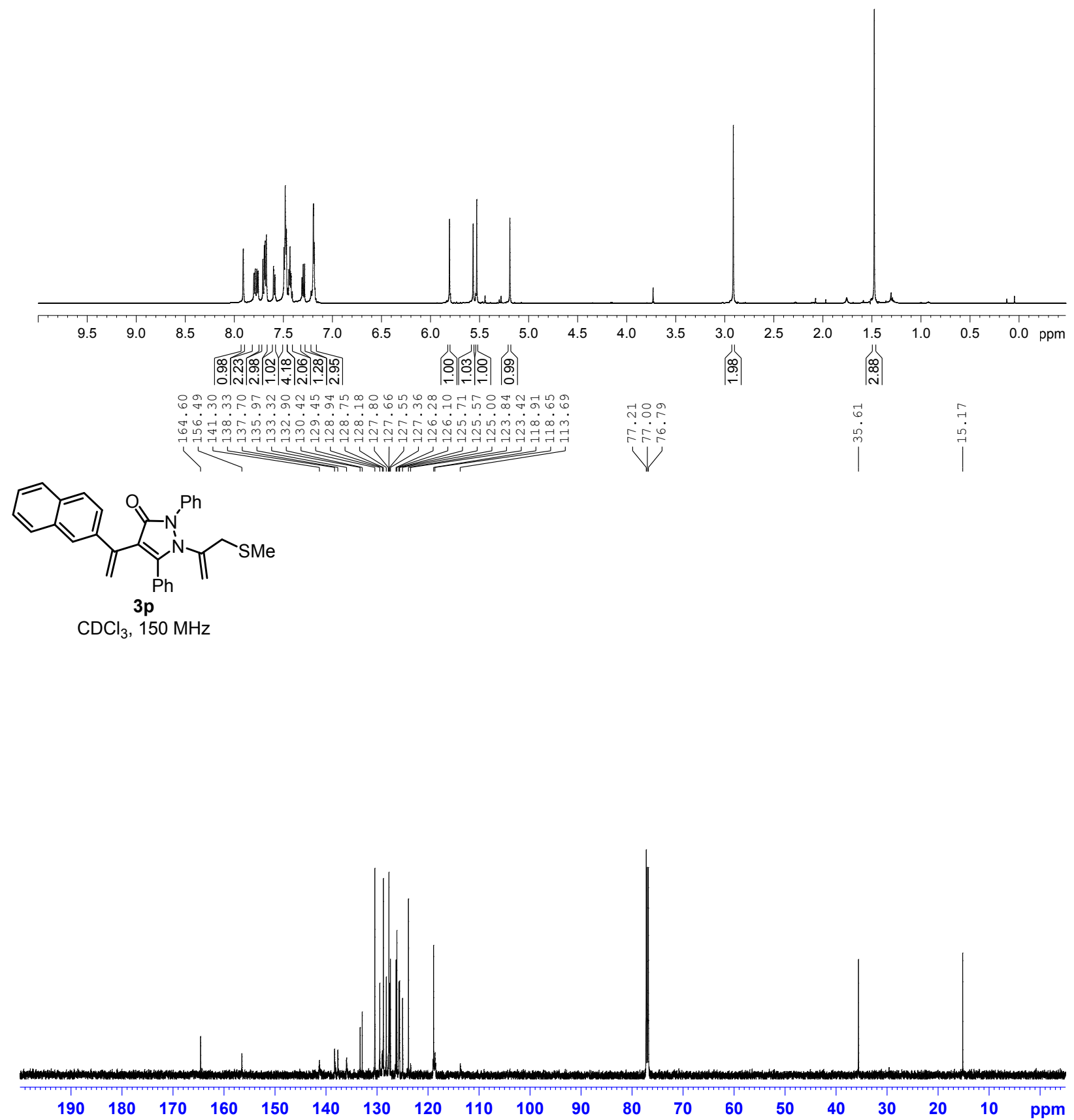


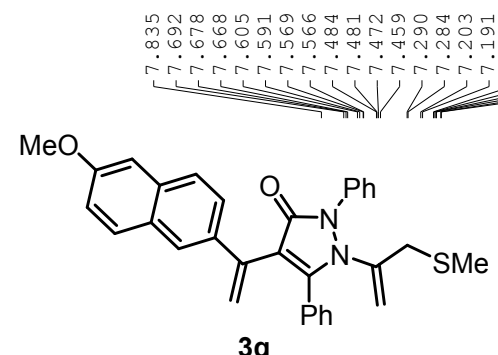

$\mathrm{CDCl}_{3}, 600 \mathrm{MHz}$
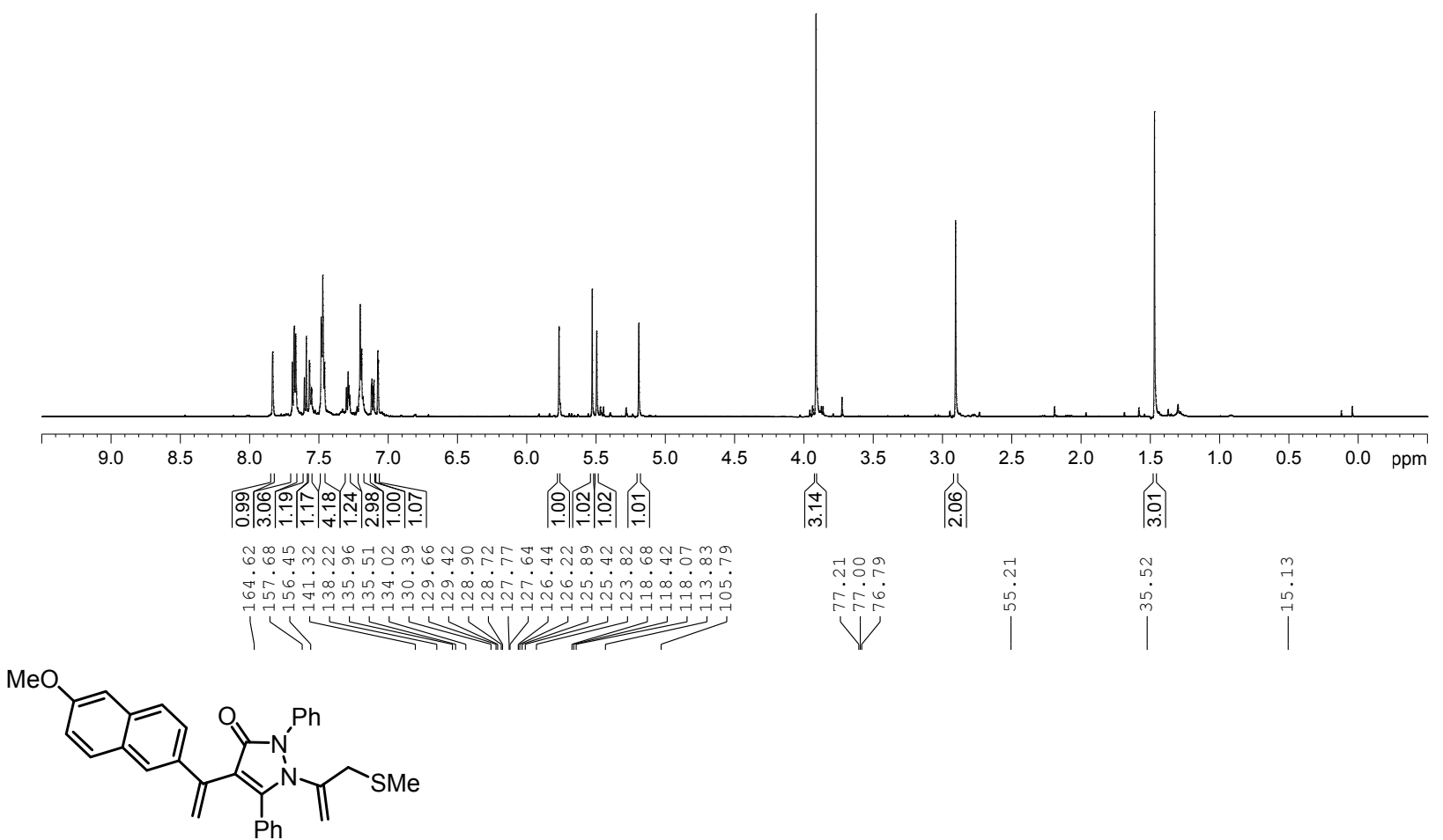

$3 q$

$\mathrm{CDCl}_{3}, 150 \mathrm{MHz}$

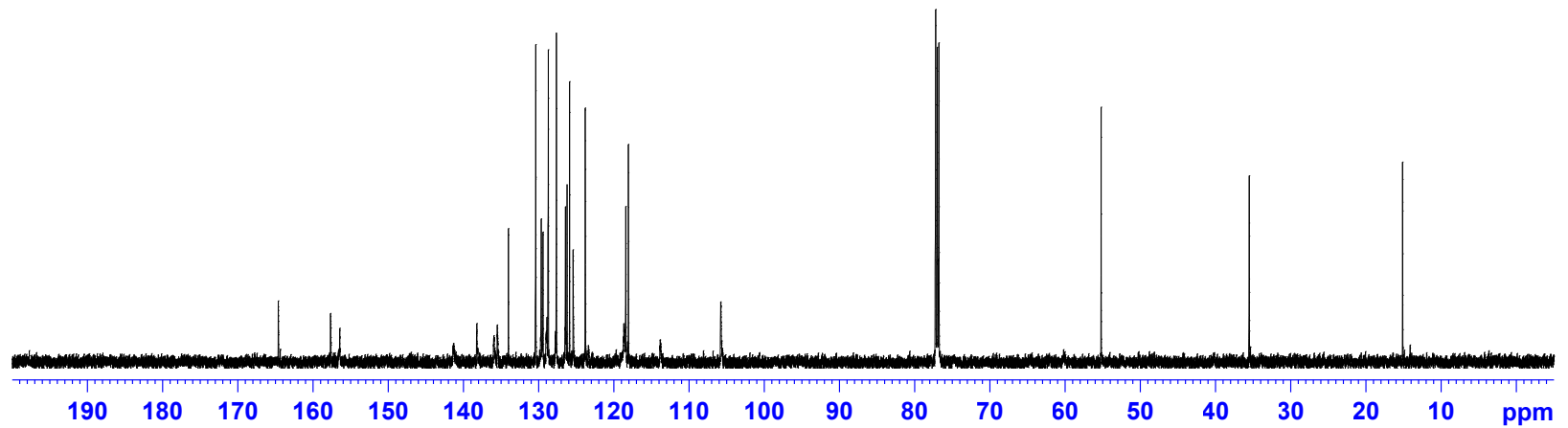



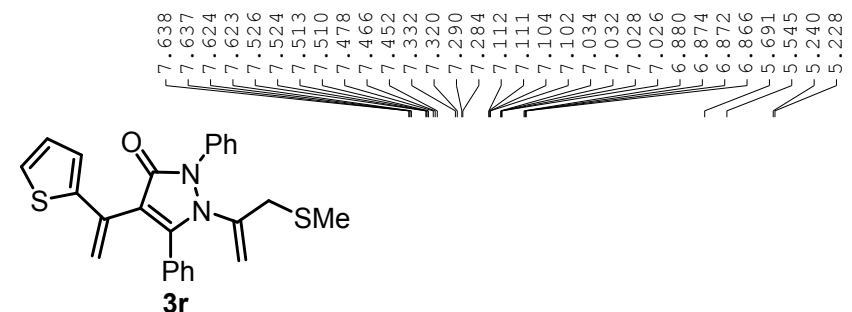

$\mathrm{CDCl}_{3}, 600 \mathrm{MHz}$
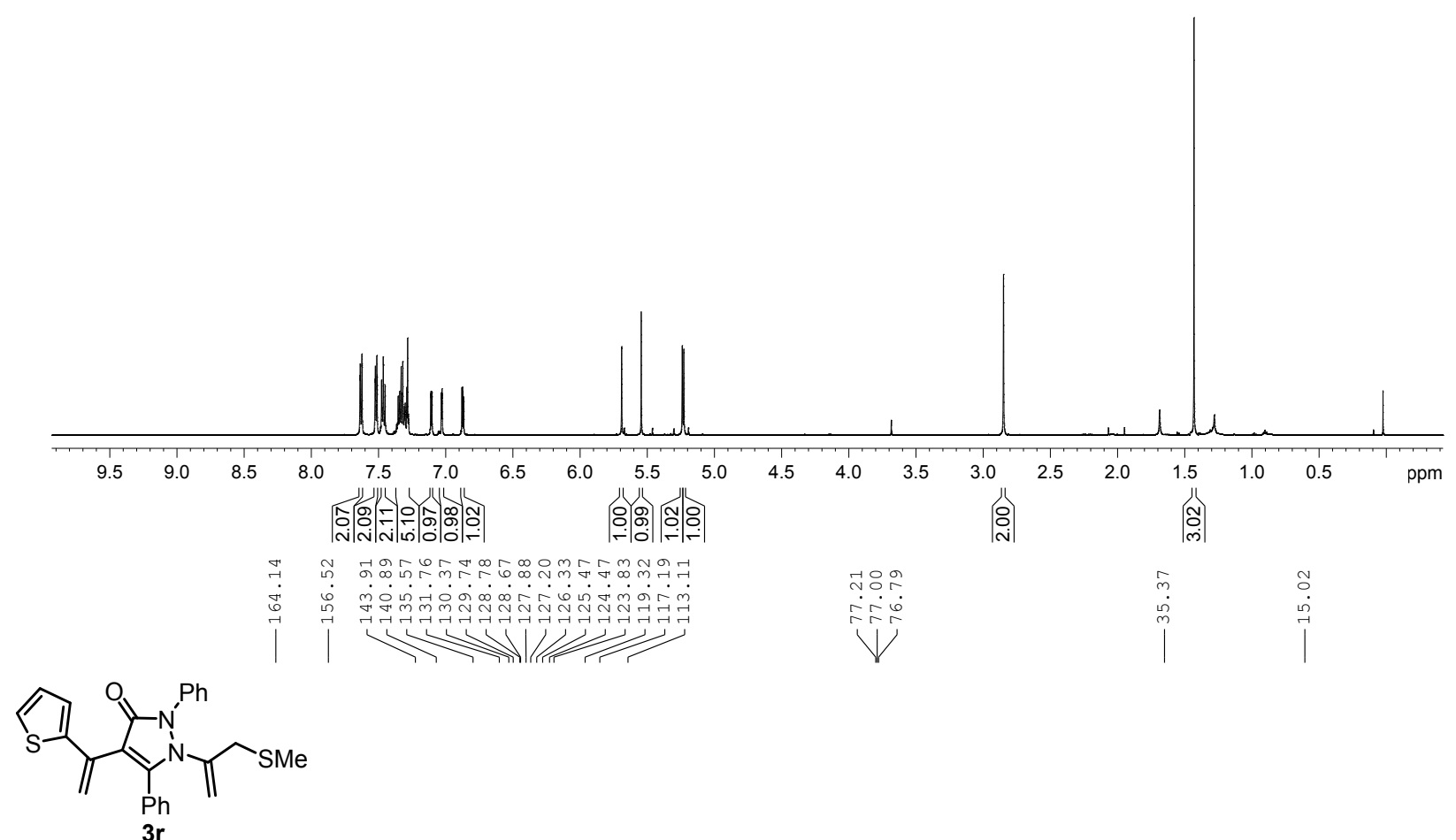

$\mathrm{CDCl}_{3}, 150 \mathrm{MHz}$

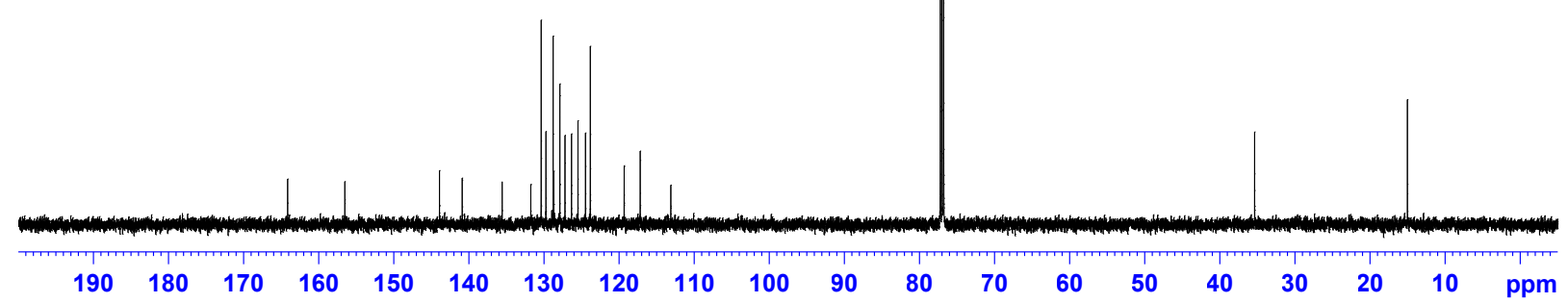



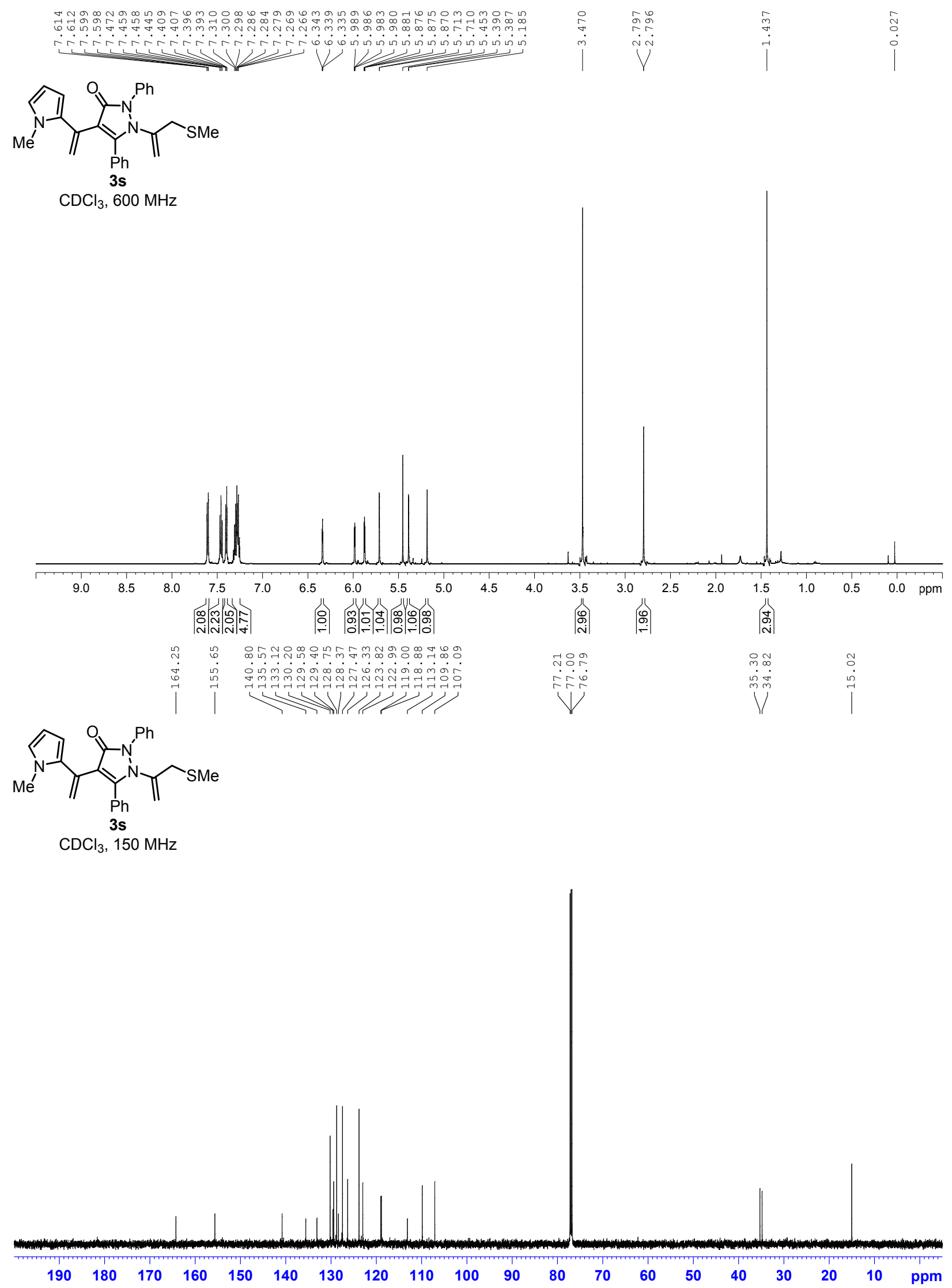

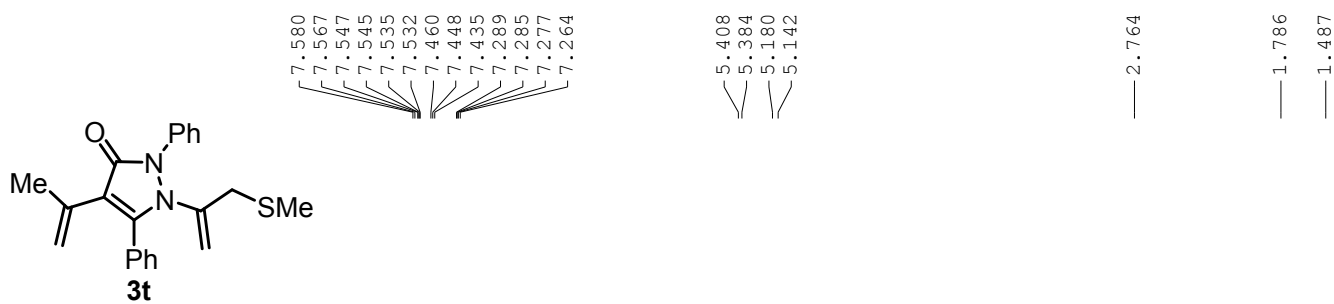

$\mathrm{CDCl}_{3}, 600 \mathrm{MHz}$

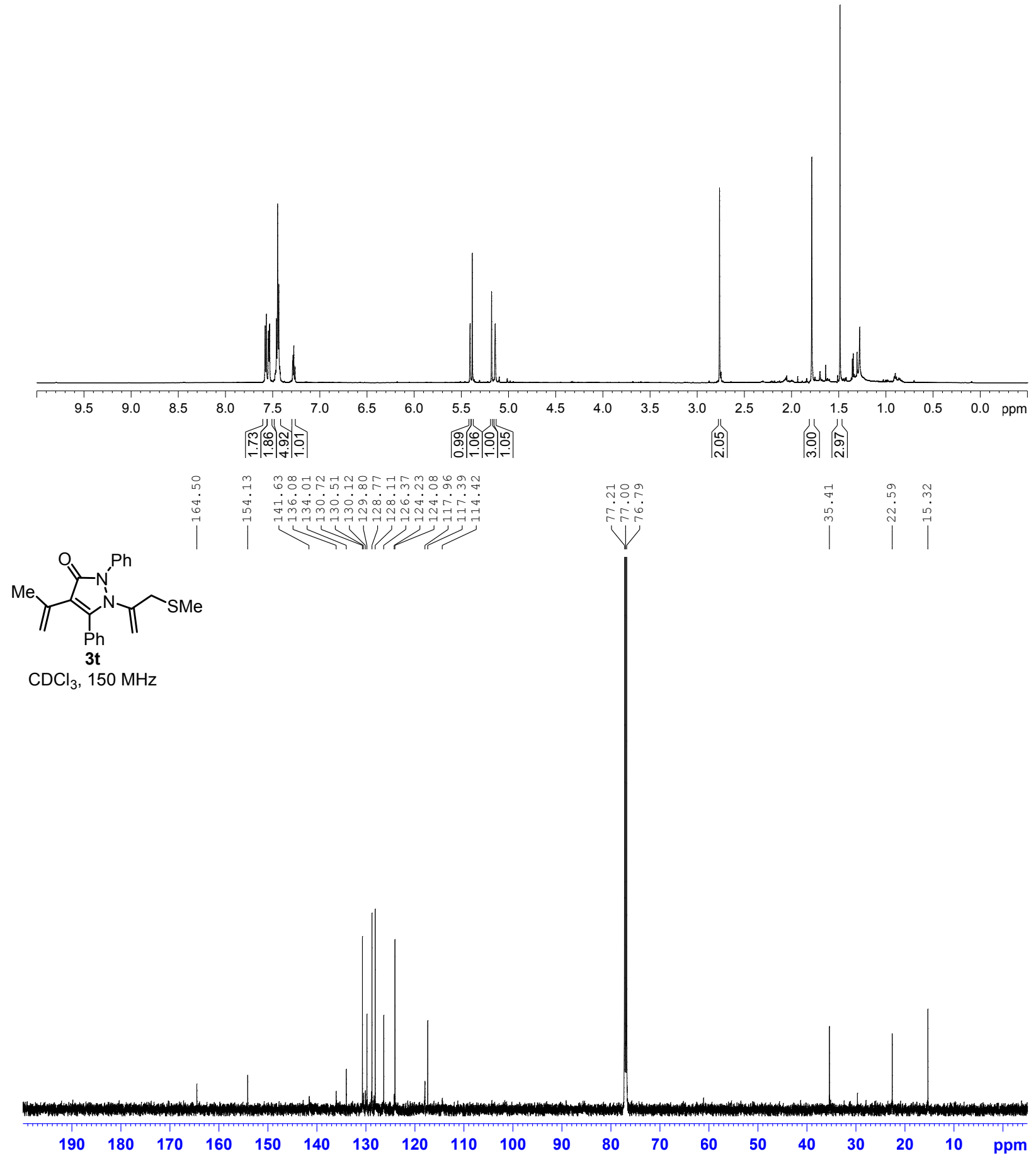



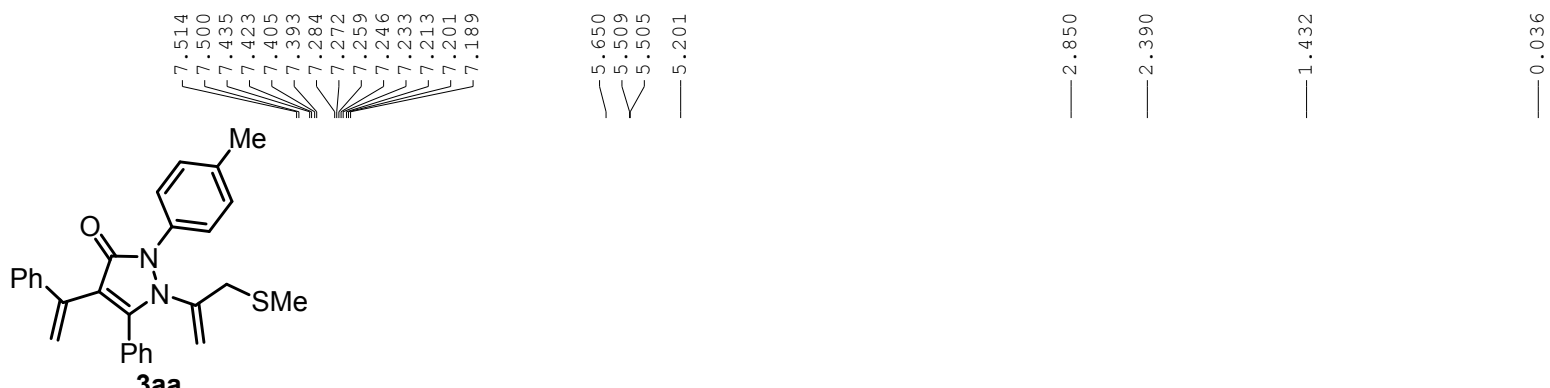

$\mathrm{CDCl}_{3}, 600 \mathrm{MHz}$
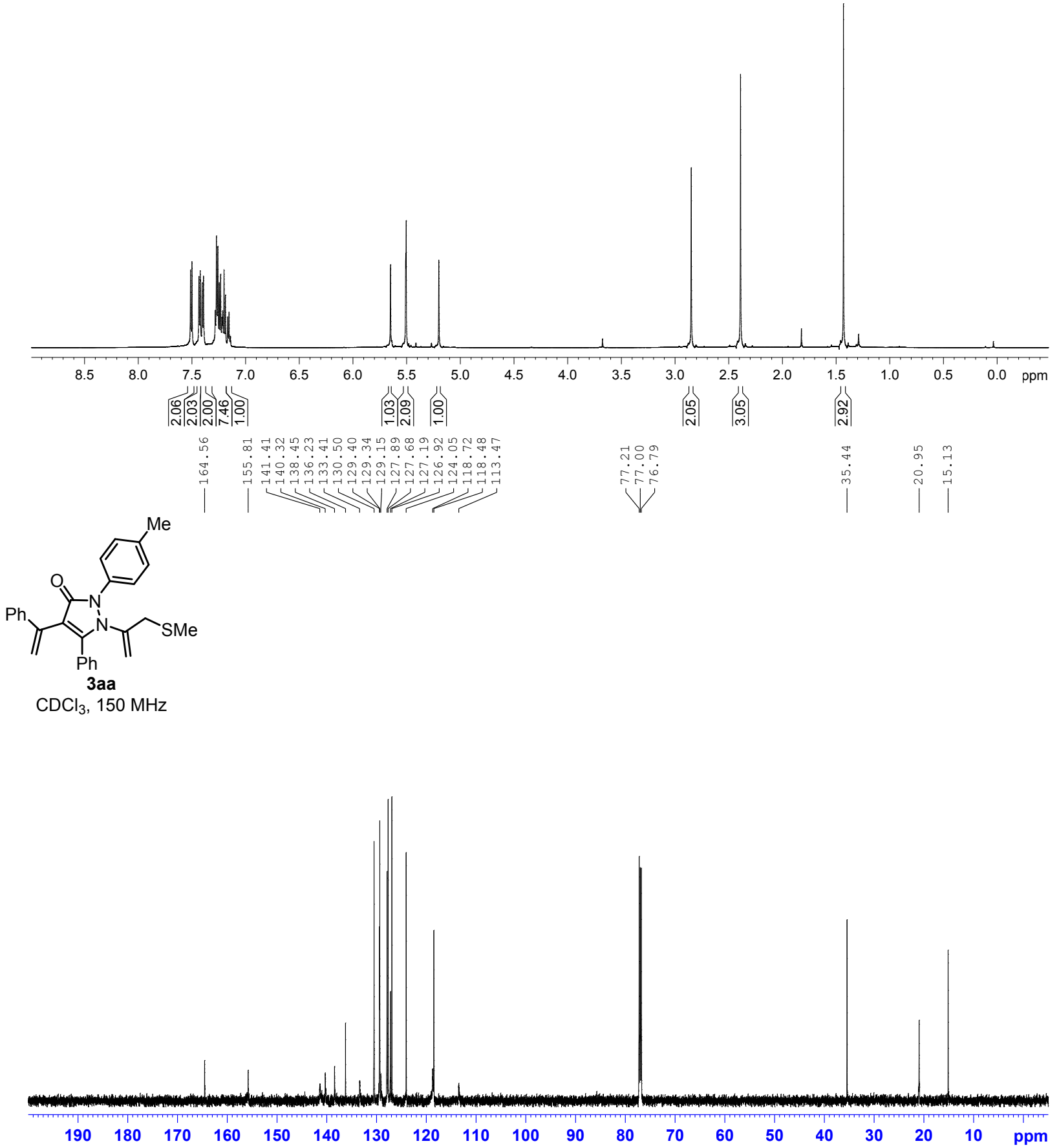

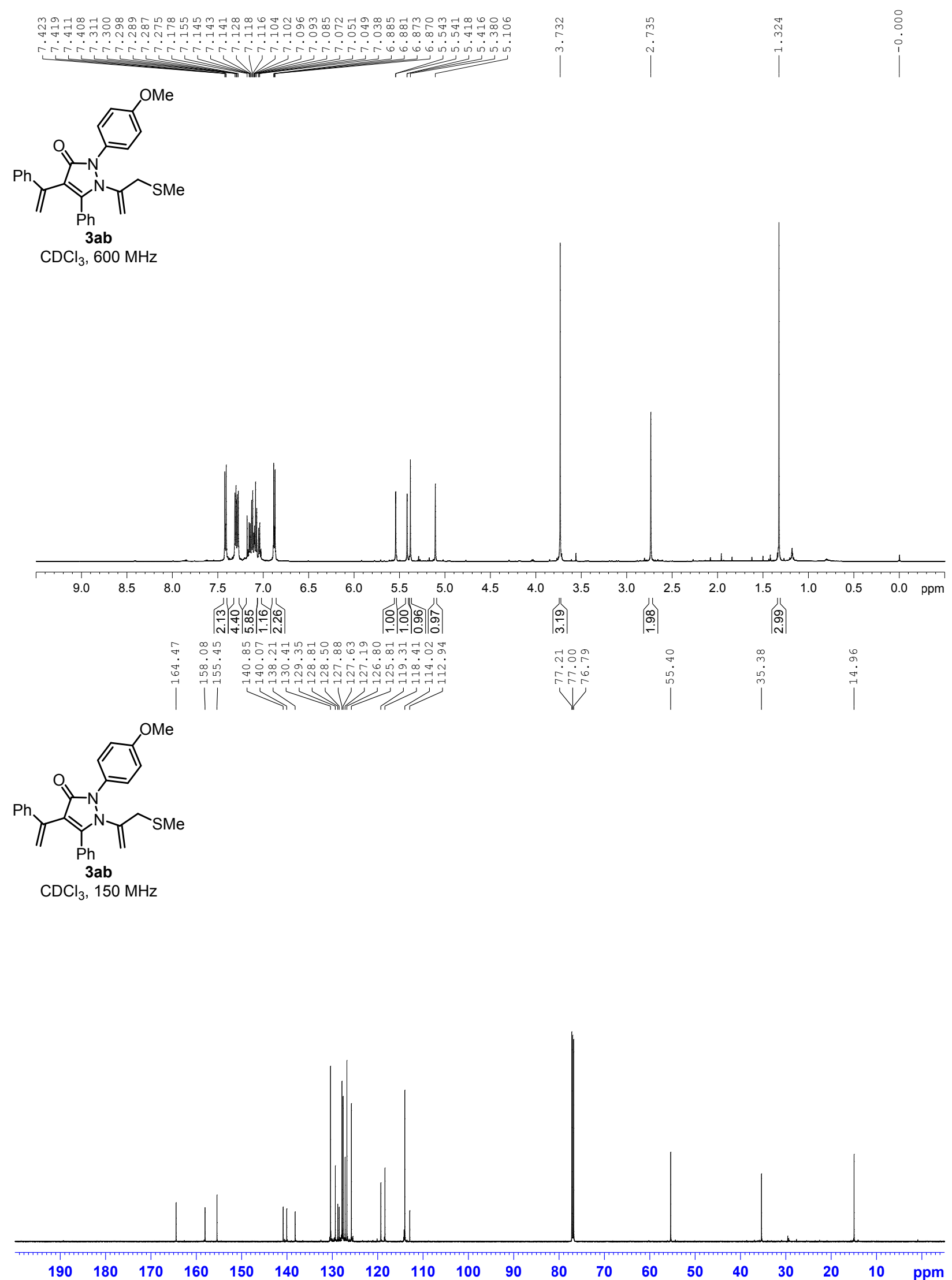

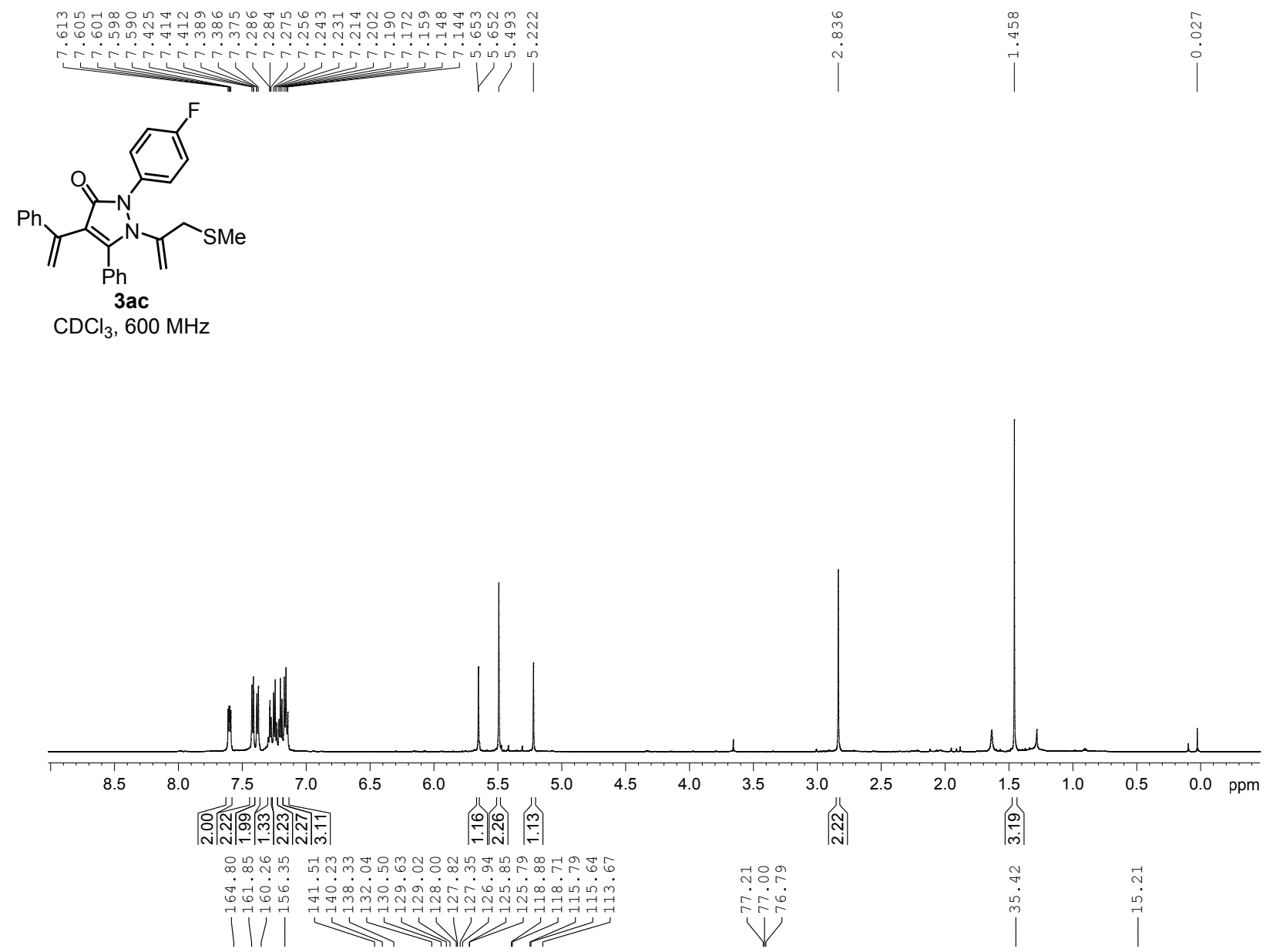<smiles>C=C(CC)c1c([Pb])n(C(=C)CC)c(=O)n1-c1ccc(F)cc1</smiles>

$\mathrm{CDCl}_{3}, 150 \mathrm{MHz}$

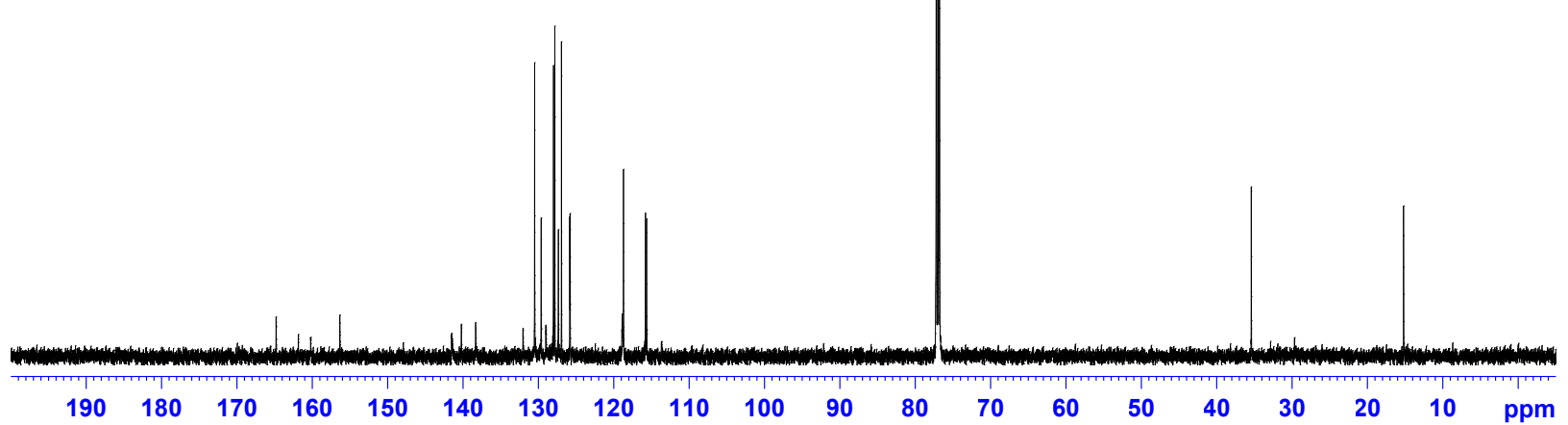



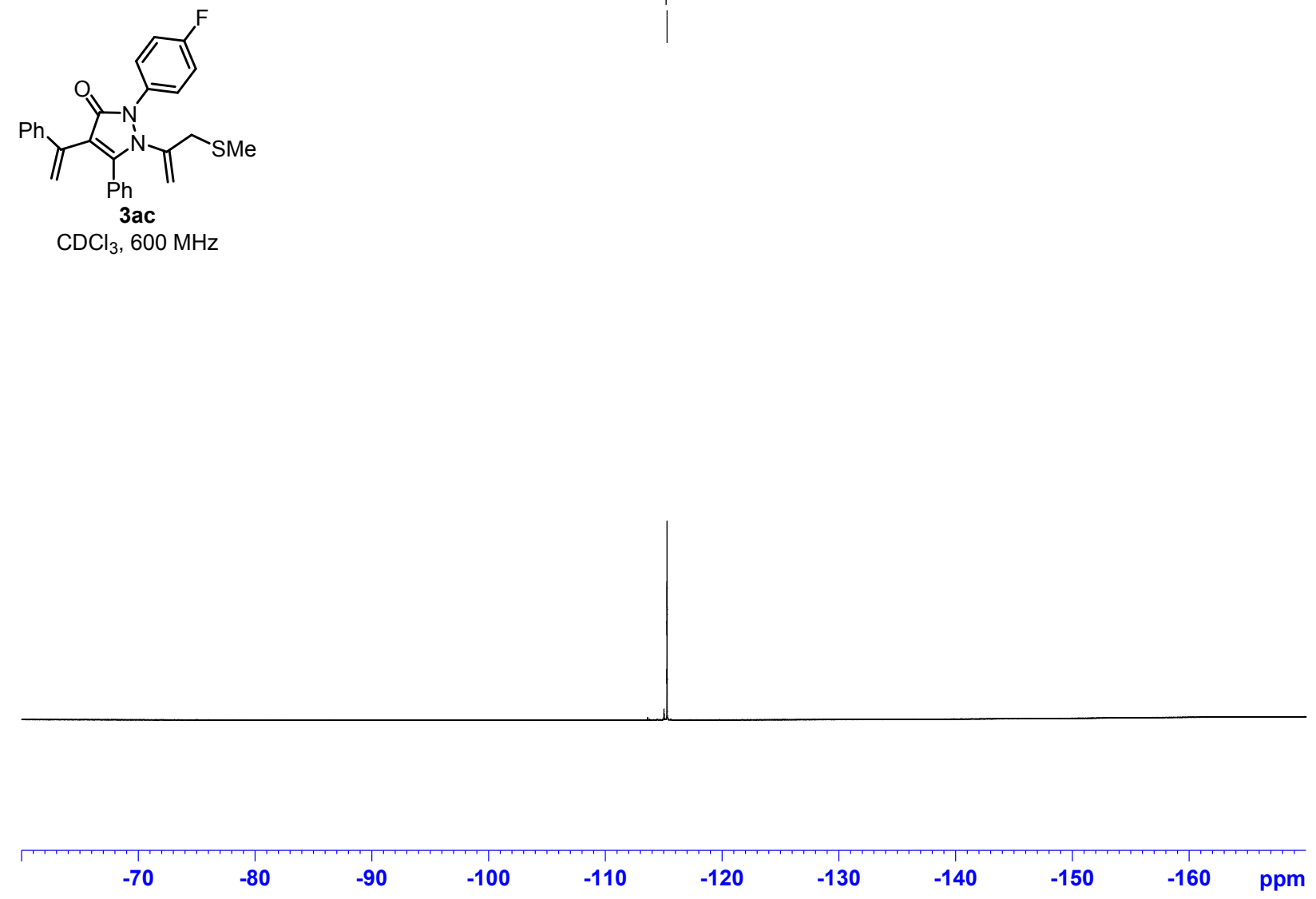


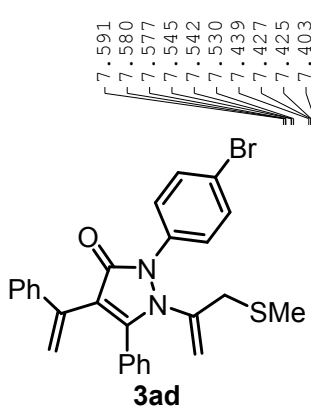

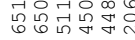

ம் ம் ம்

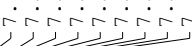

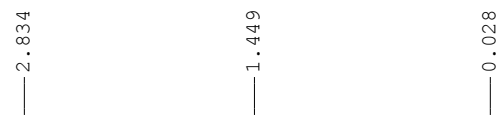

$\mathrm{CDCl}_{3}, 600 \mathrm{MHz}$
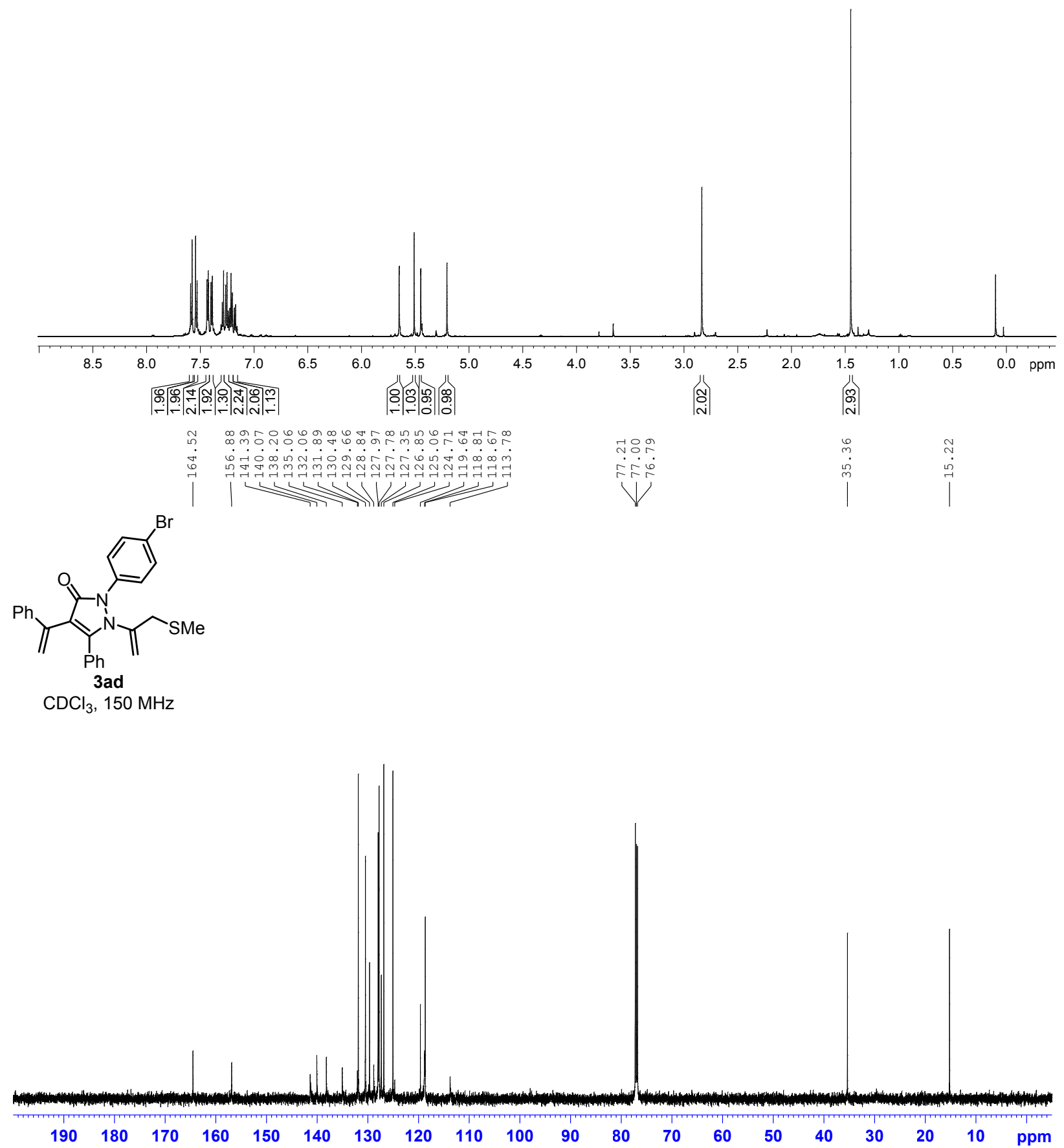


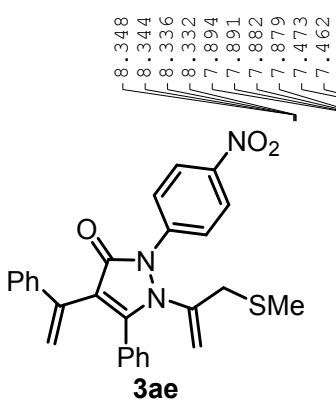

$\mathrm{CDCl}_{3}, 600 \mathrm{MHz}$

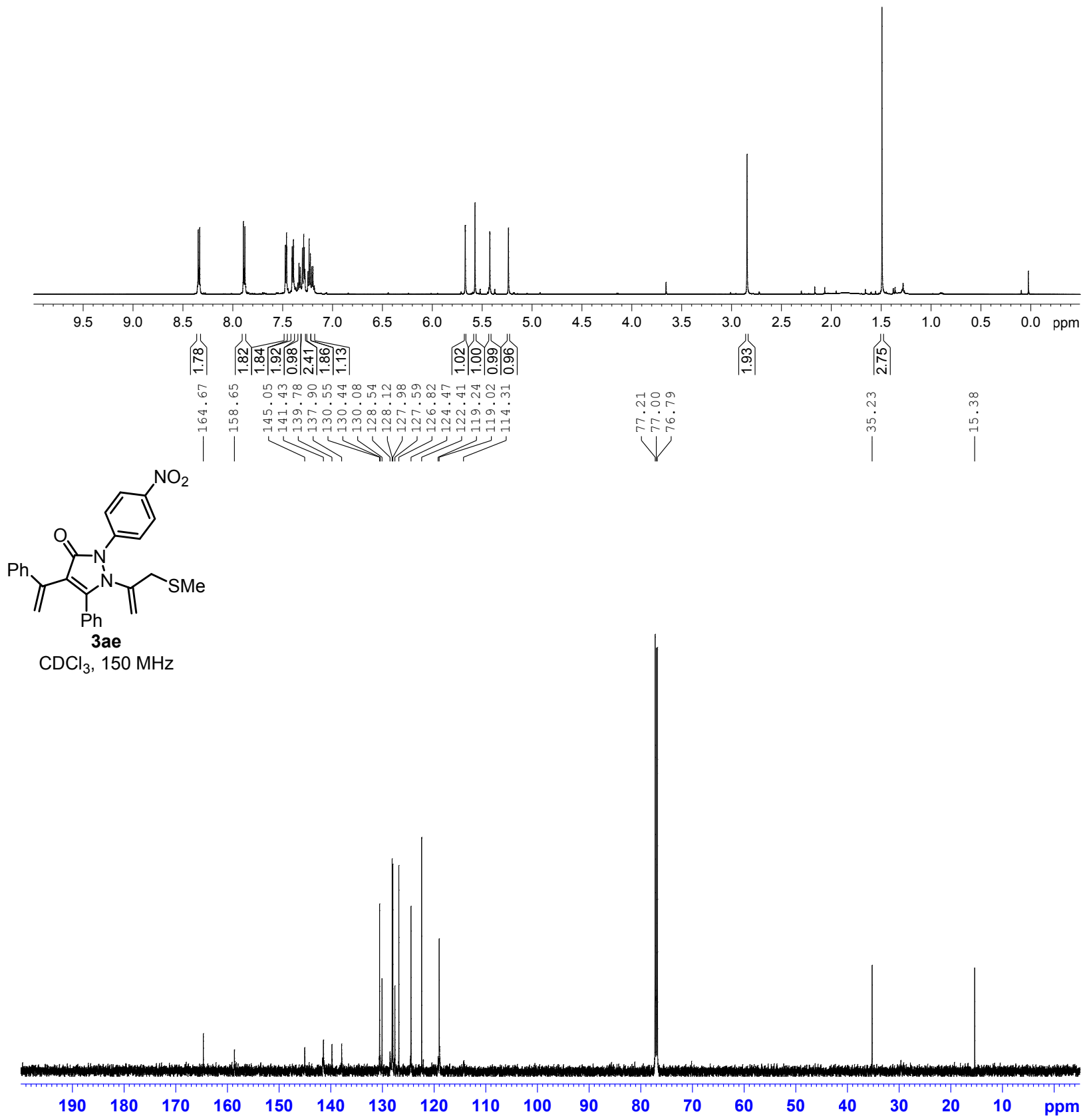




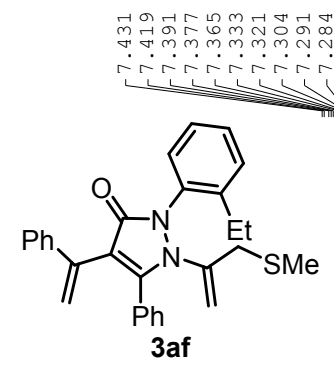

$\mathrm{CDCl}_{3}, 600 \mathrm{MHz}$

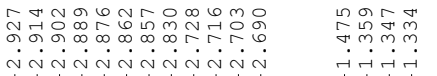

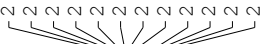

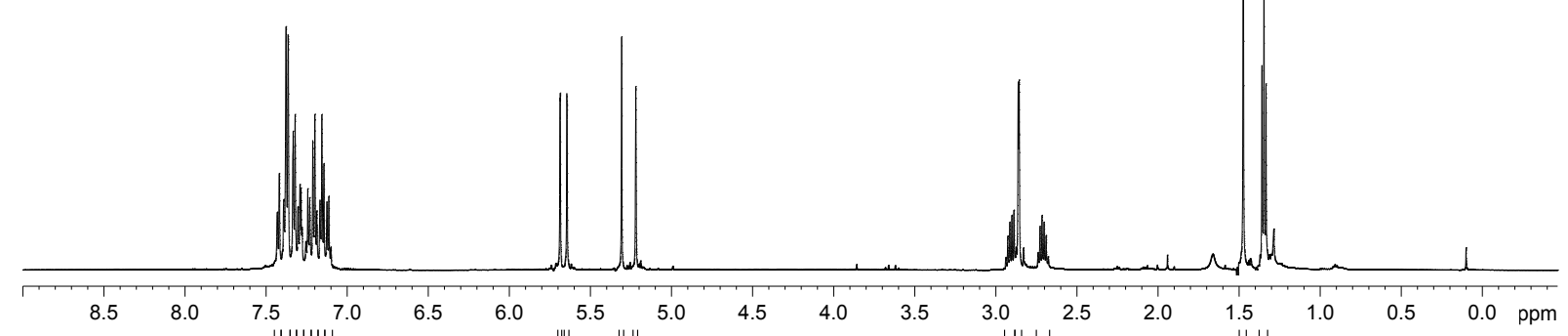

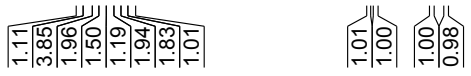

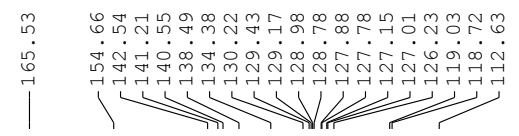<smiles>C=C(C(=C)n1c(-c2ccccc2)c(C(=C)C)c(=O)n1-c1ccccc1)c1ccccc1</smiles>

3 af

$\mathrm{CDCl}_{3}, 150 \mathrm{MHz}$

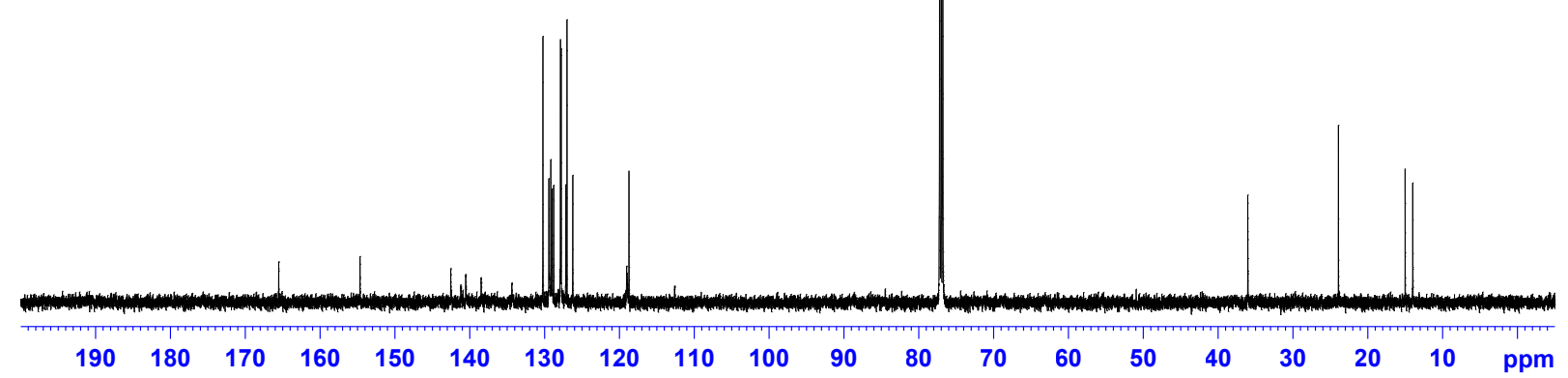



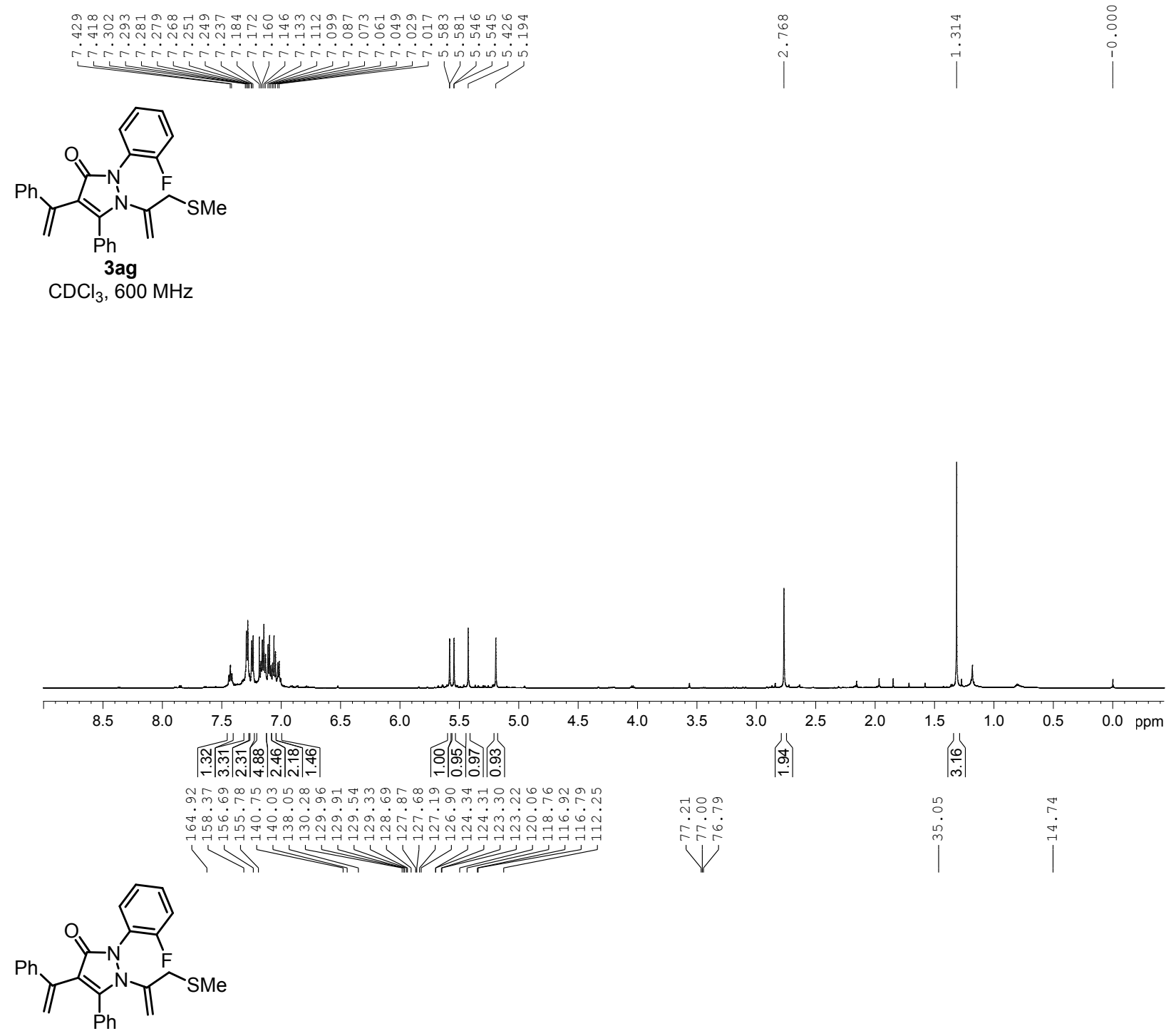

3 ag

$\mathrm{CDCl}_{3}, 150 \mathrm{MHz}$

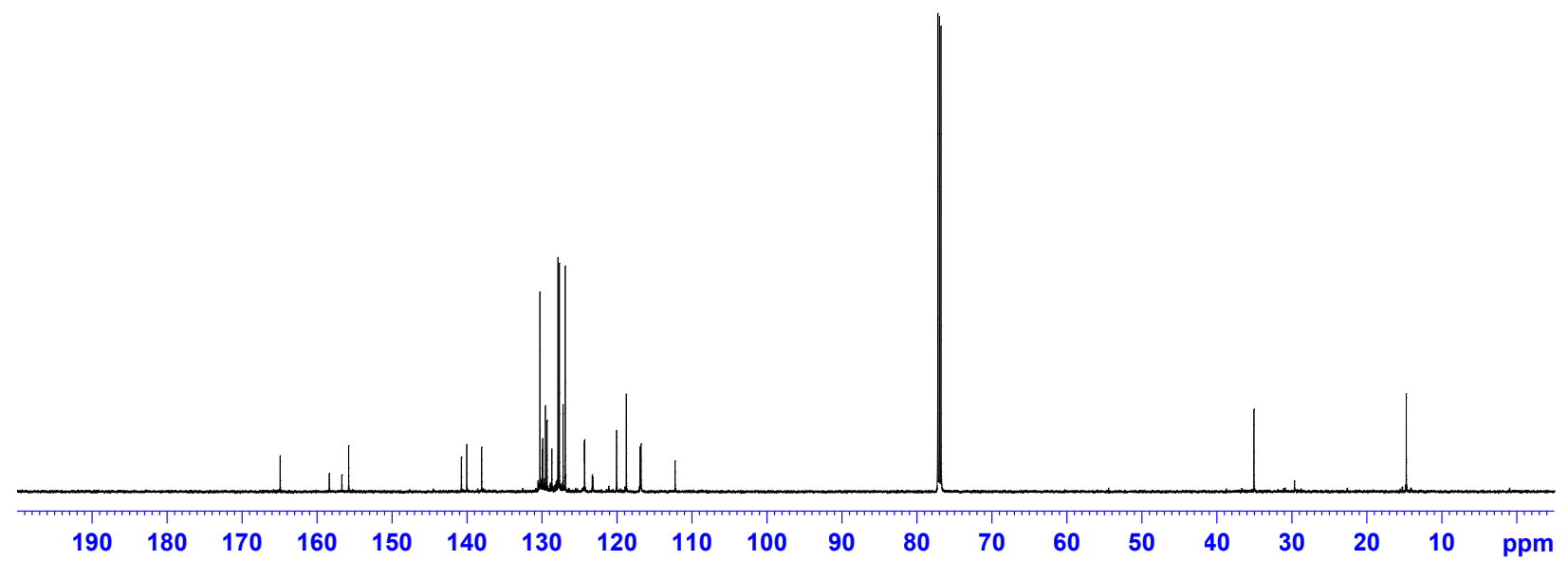



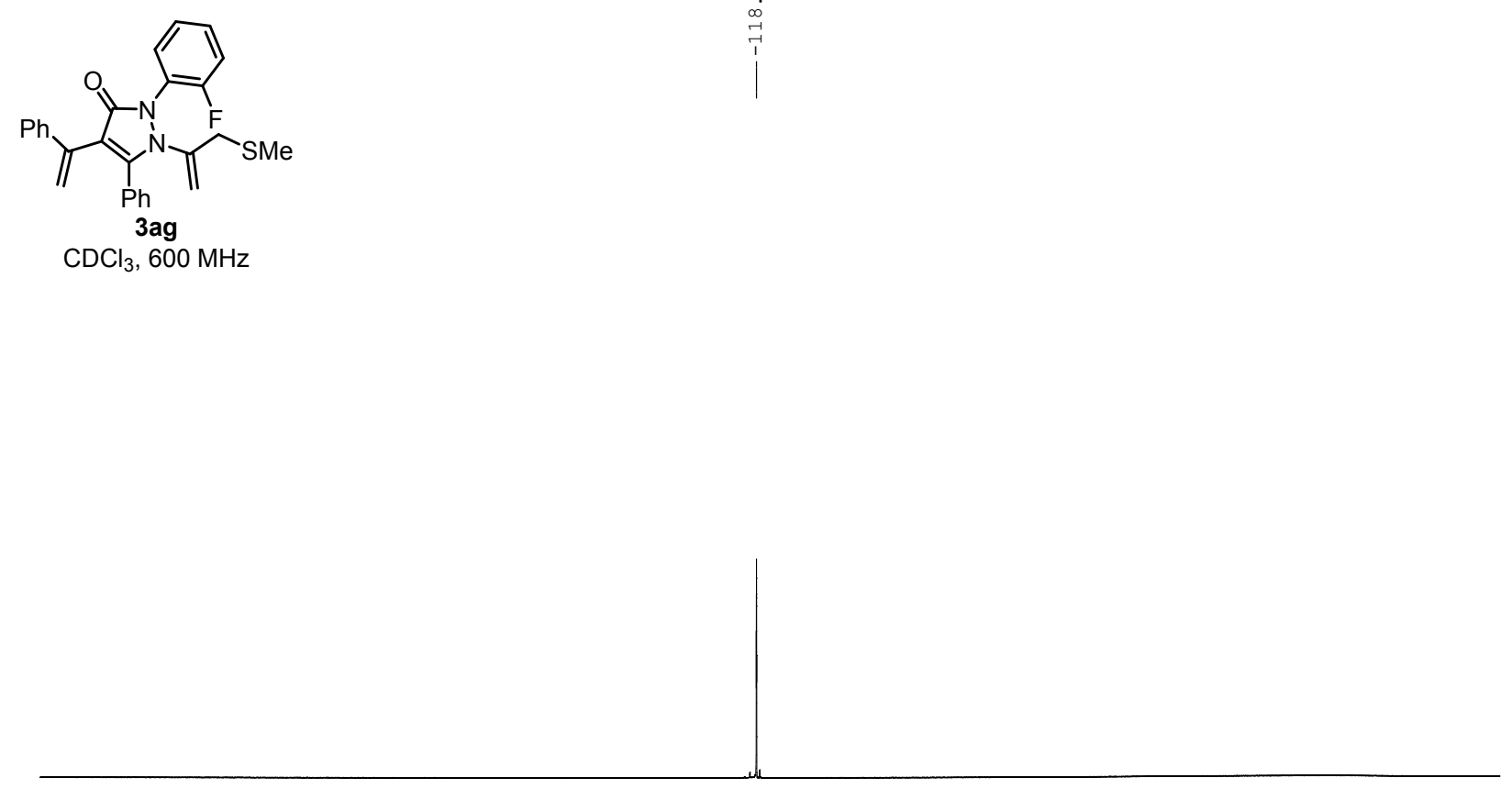

\begin{tabular}{|c|c|c|c|c|c|c|c|c|c|c|c|}
\hline-70 & -80 & -90 & -100 & -110 & -120 & -130 & -140 & -150 & -160 & -170 & ppm \\
\hline
\end{tabular}




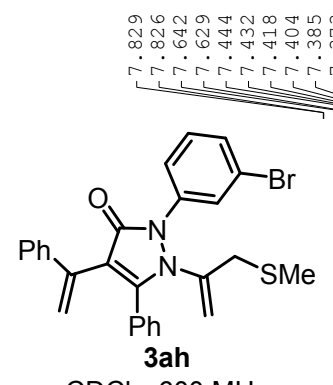

$\mathrm{CDCl}_{3}, 600 \mathrm{MHz}$

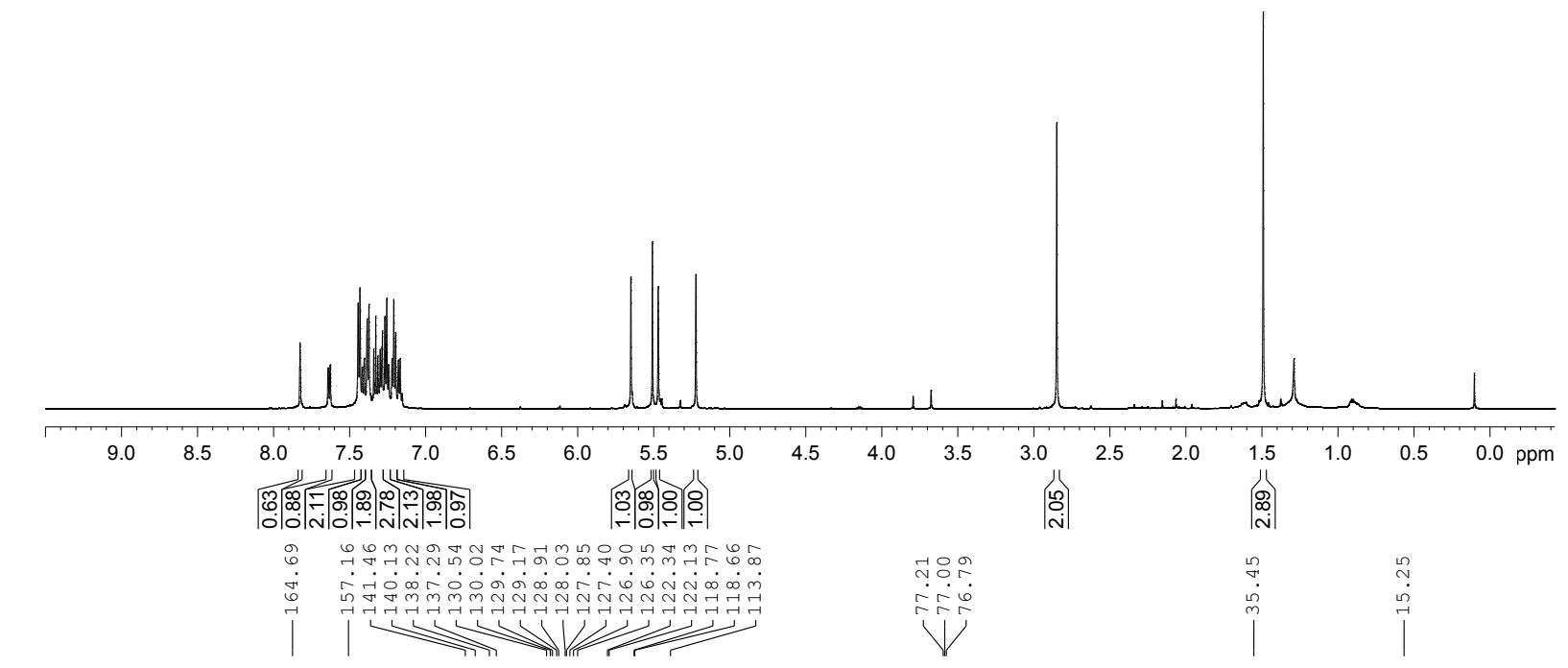<smiles>C=C(C(=C)n1c(-c2ccccc2)c(C(=C)CC)c(=O)n1-c1cccc(Br)c1)c1ccccc1</smiles>

3ah

$\mathrm{CDCl}_{3}, 150 \mathrm{MHz}$

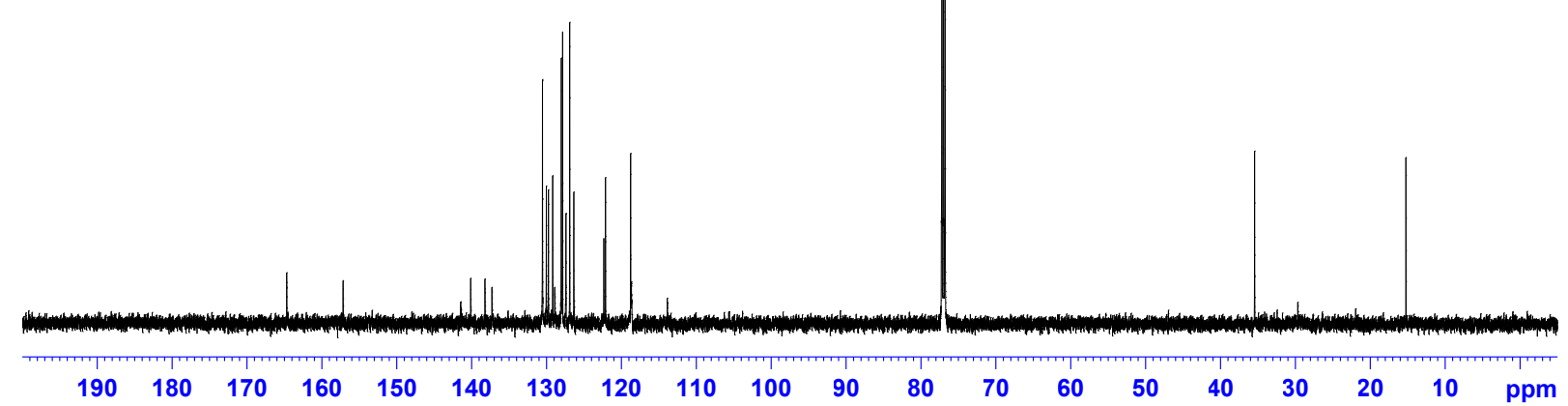




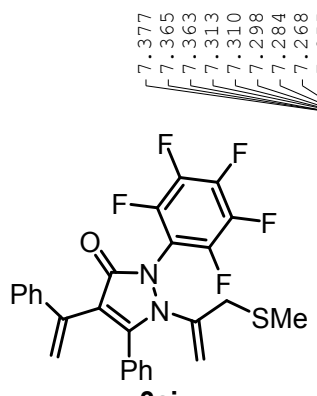

3ai

$\mathrm{CDCl}_{3}, 600 \mathrm{MHz}$
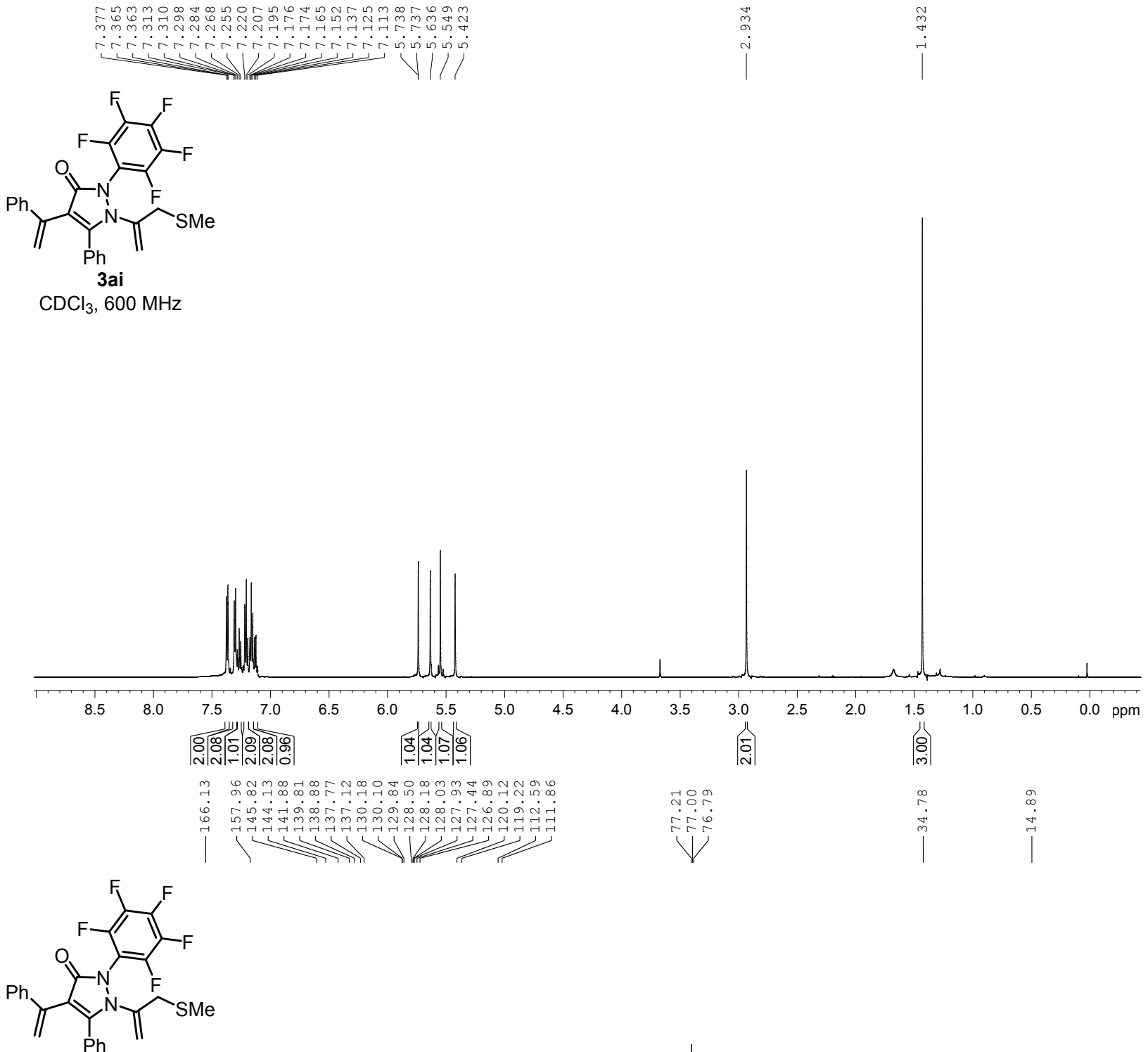

$3 a i$

$\mathrm{CDCl}_{3}, 150 \mathrm{MHz}$

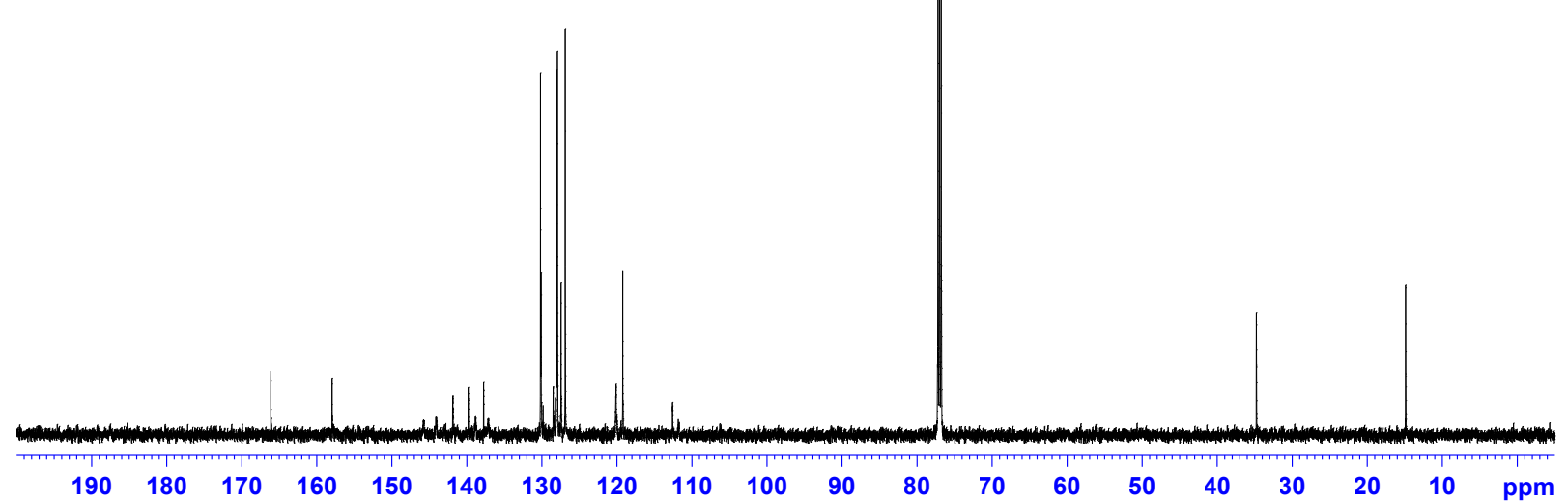




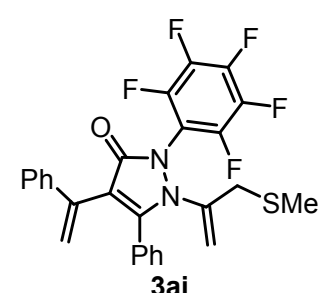

$\mathrm{CDCl}_{3}, 600 \mathrm{MHz}$

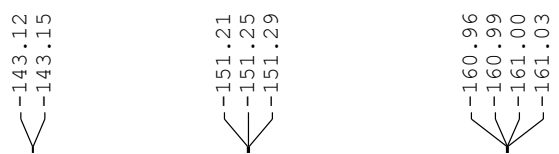

엄

V

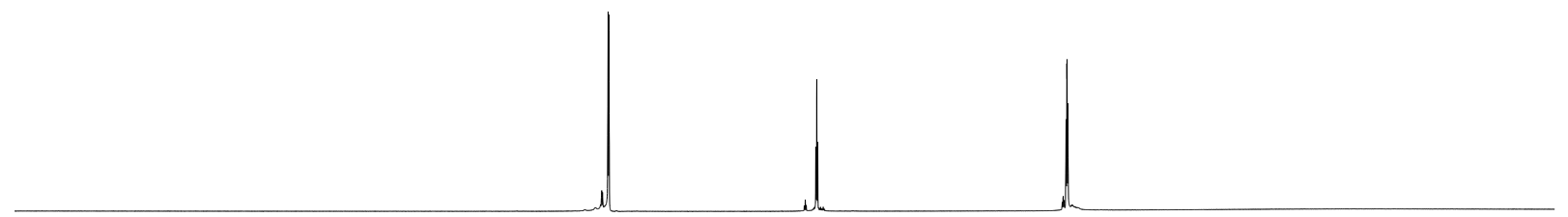

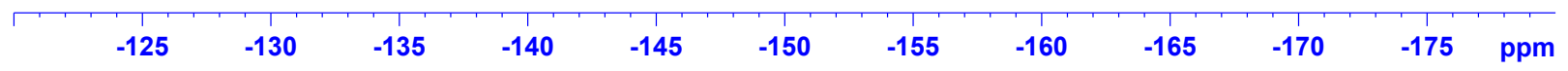




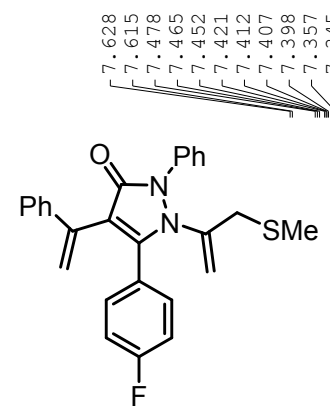

3aj
$\mathrm{CDCl}_{3}, 600 \mathrm{MHz}$
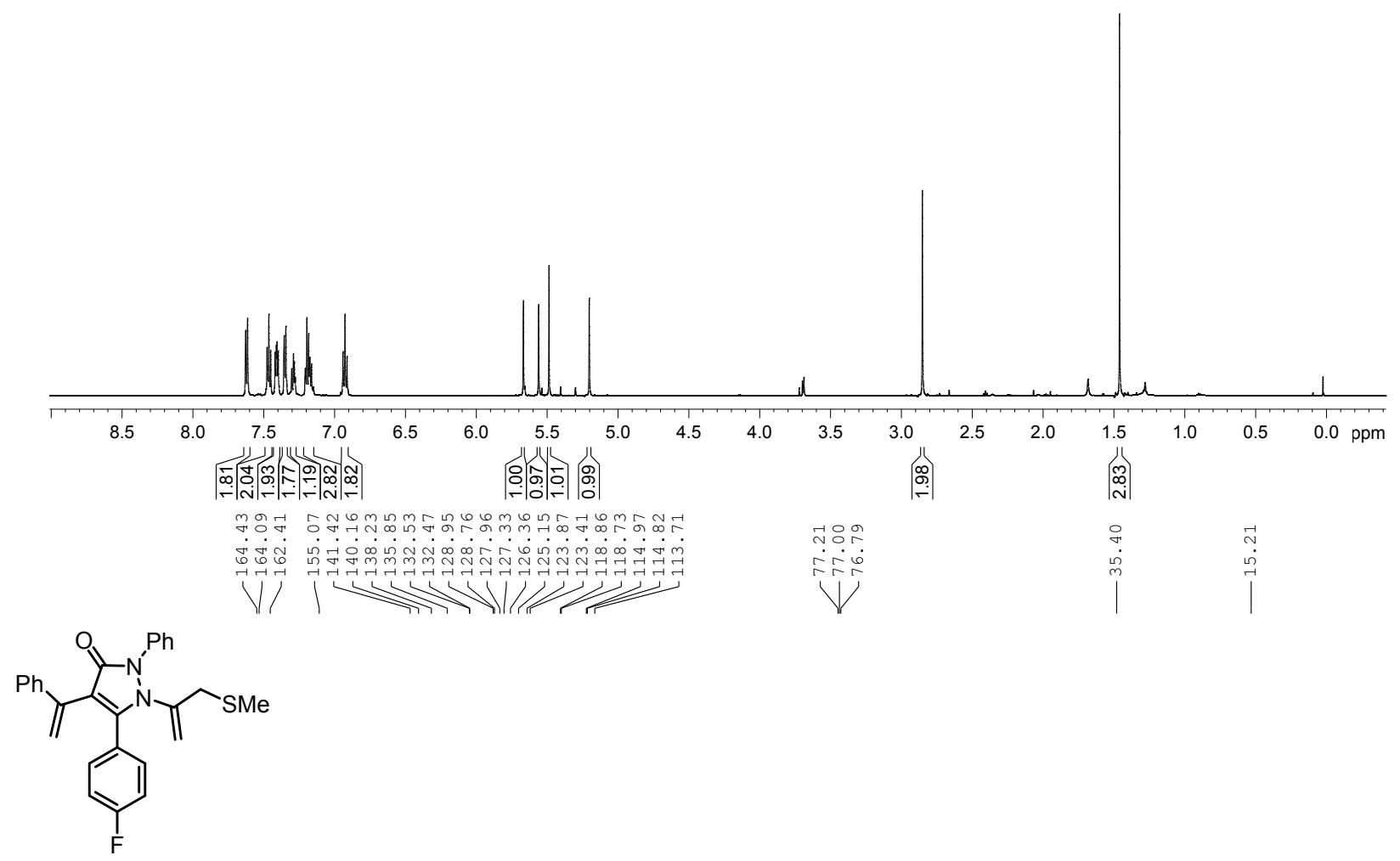

3aj

$\mathrm{CDCl}_{3}, 150 \mathrm{MHz}$

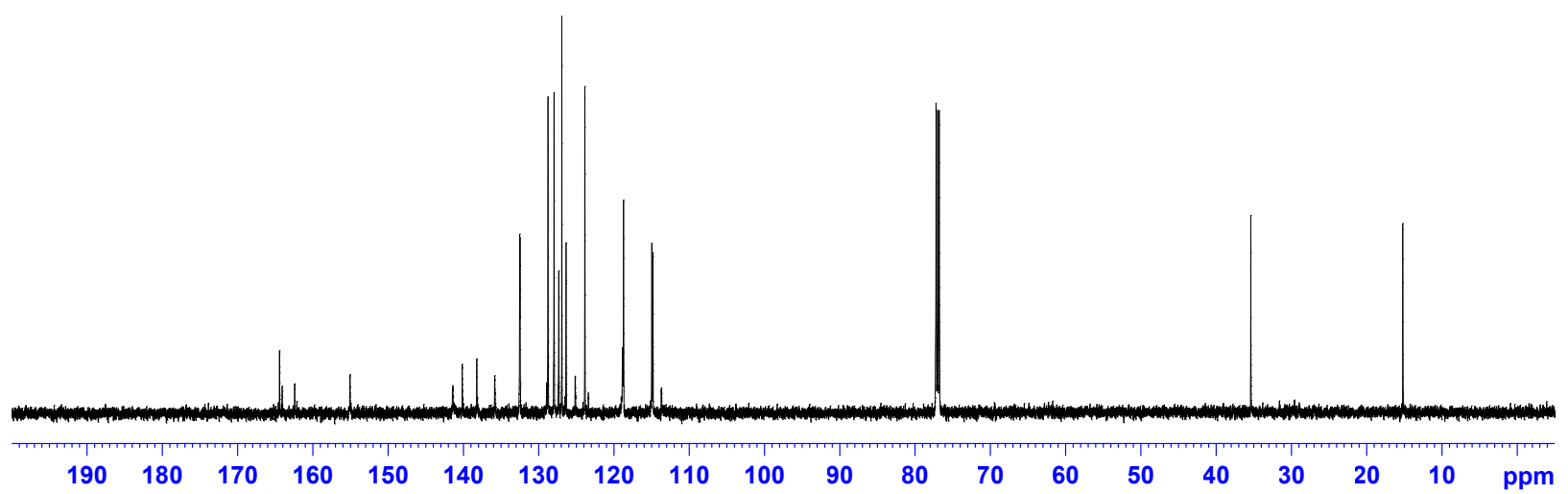



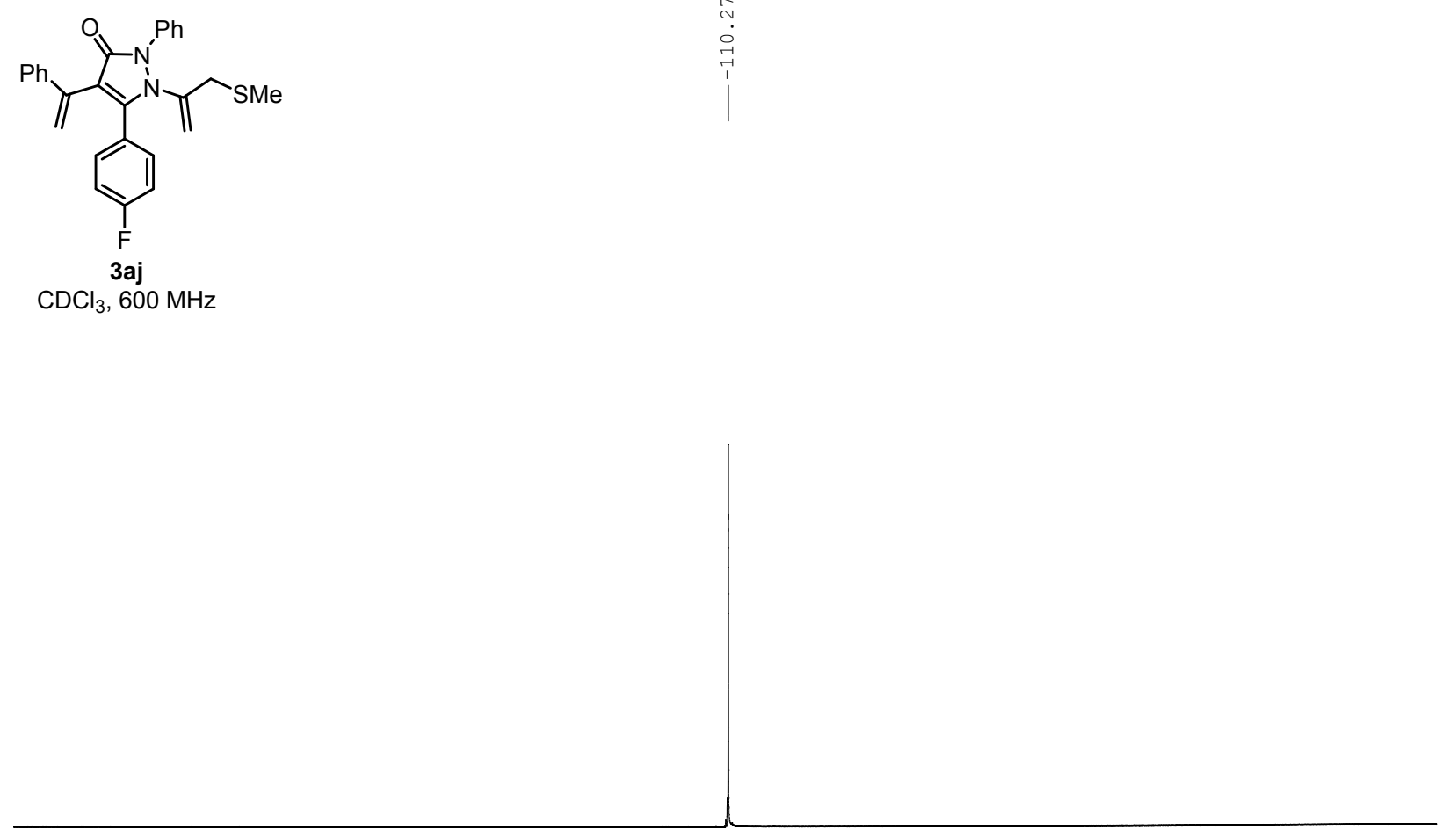

\begin{tabular}{rlllllllllll}
\hline-60 & -70 & -80 & -90 & -100 & -110 & -120 & -130 & -140 & -150 & -160 & ppm
\end{tabular}




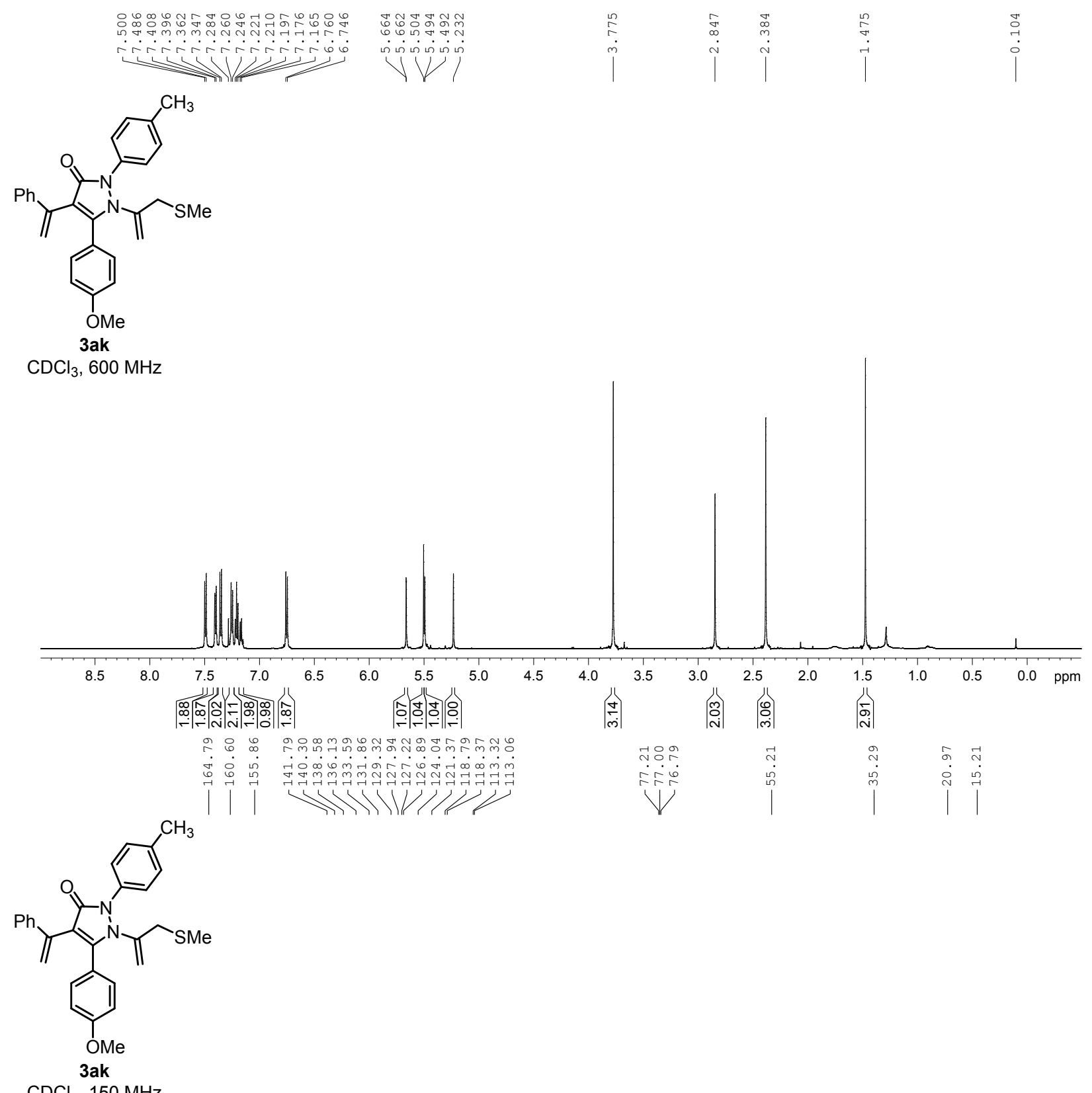

$\mathrm{CDCl}_{3}, 150 \mathrm{MHz}$

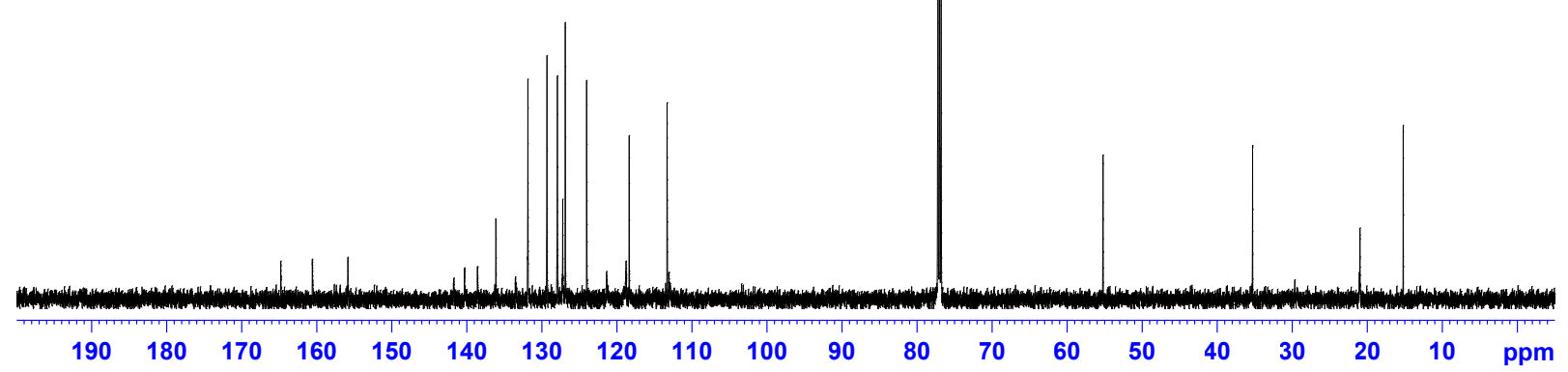




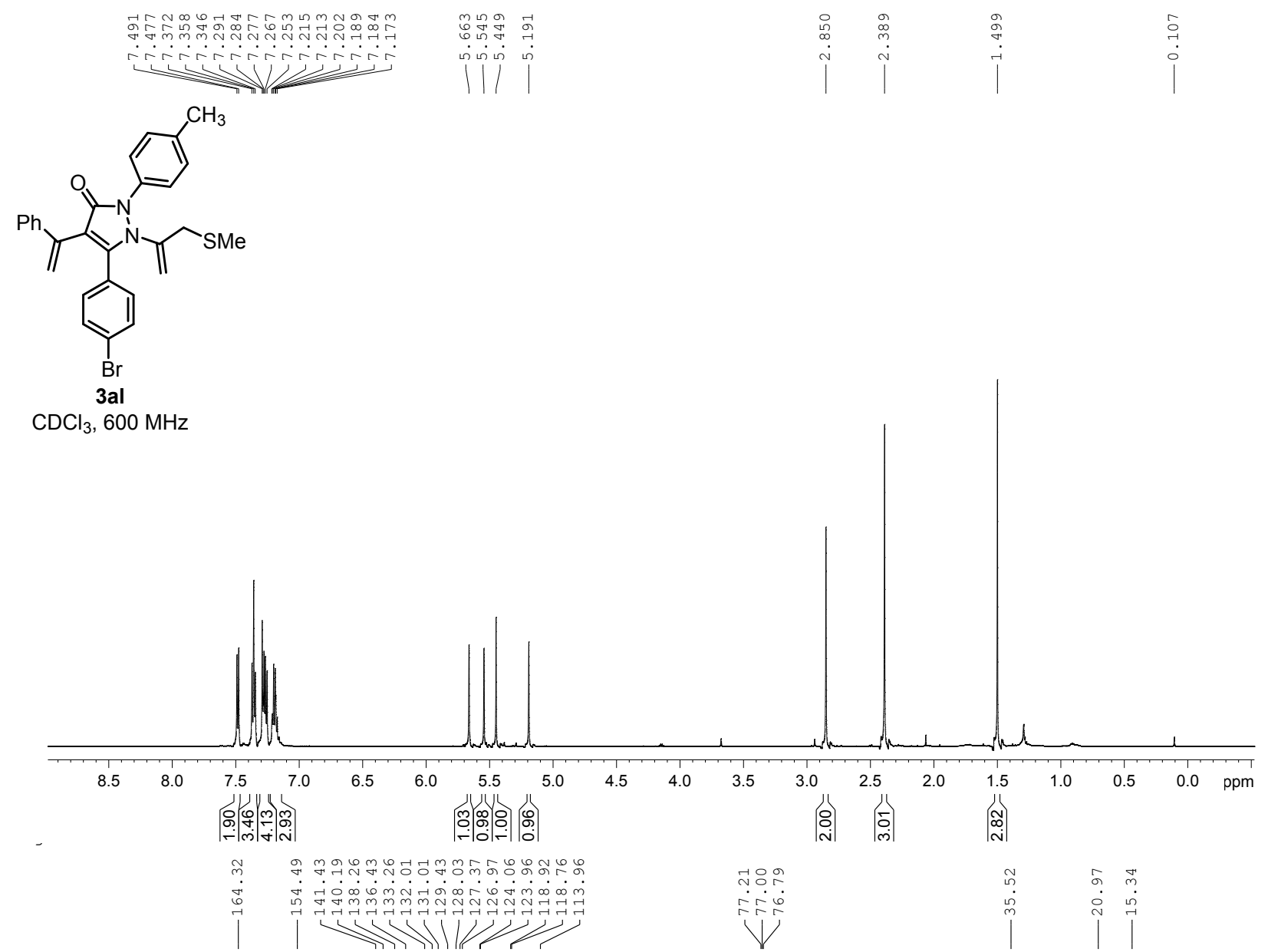<smiles>C=C(CC)c1c(-c2ccccc2)c(=O)n(-c2ccc(C)cc2)n1C(=C)CC</smiles>

$\mathrm{CDCl}_{3}, 150 \mathrm{MHz}$

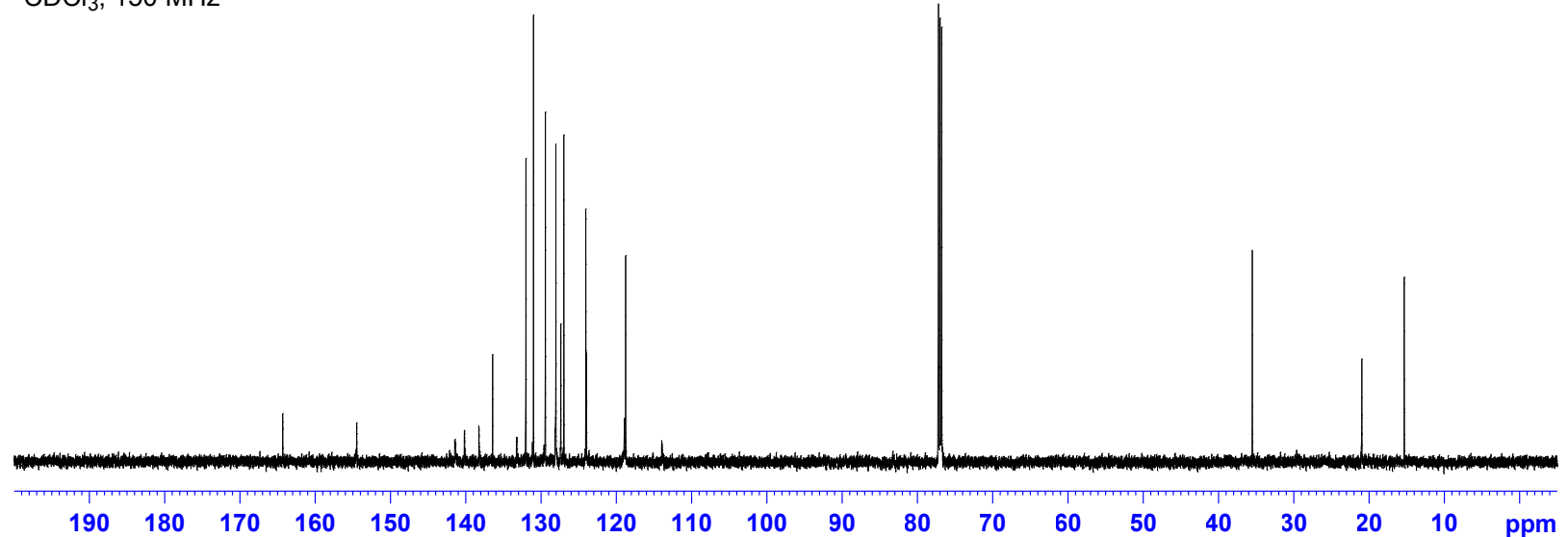



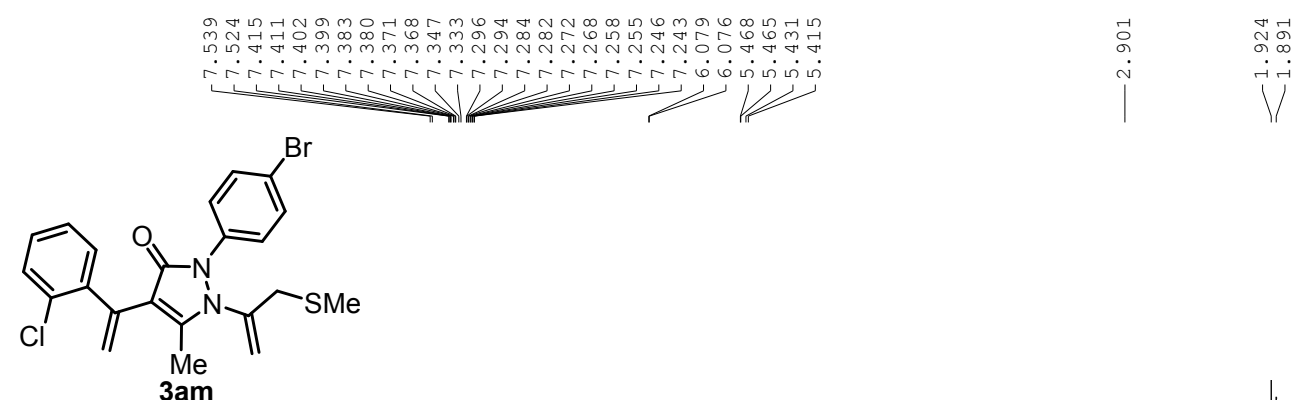

$\mathrm{CDCl}_{3}, 600 \mathrm{MHz}$
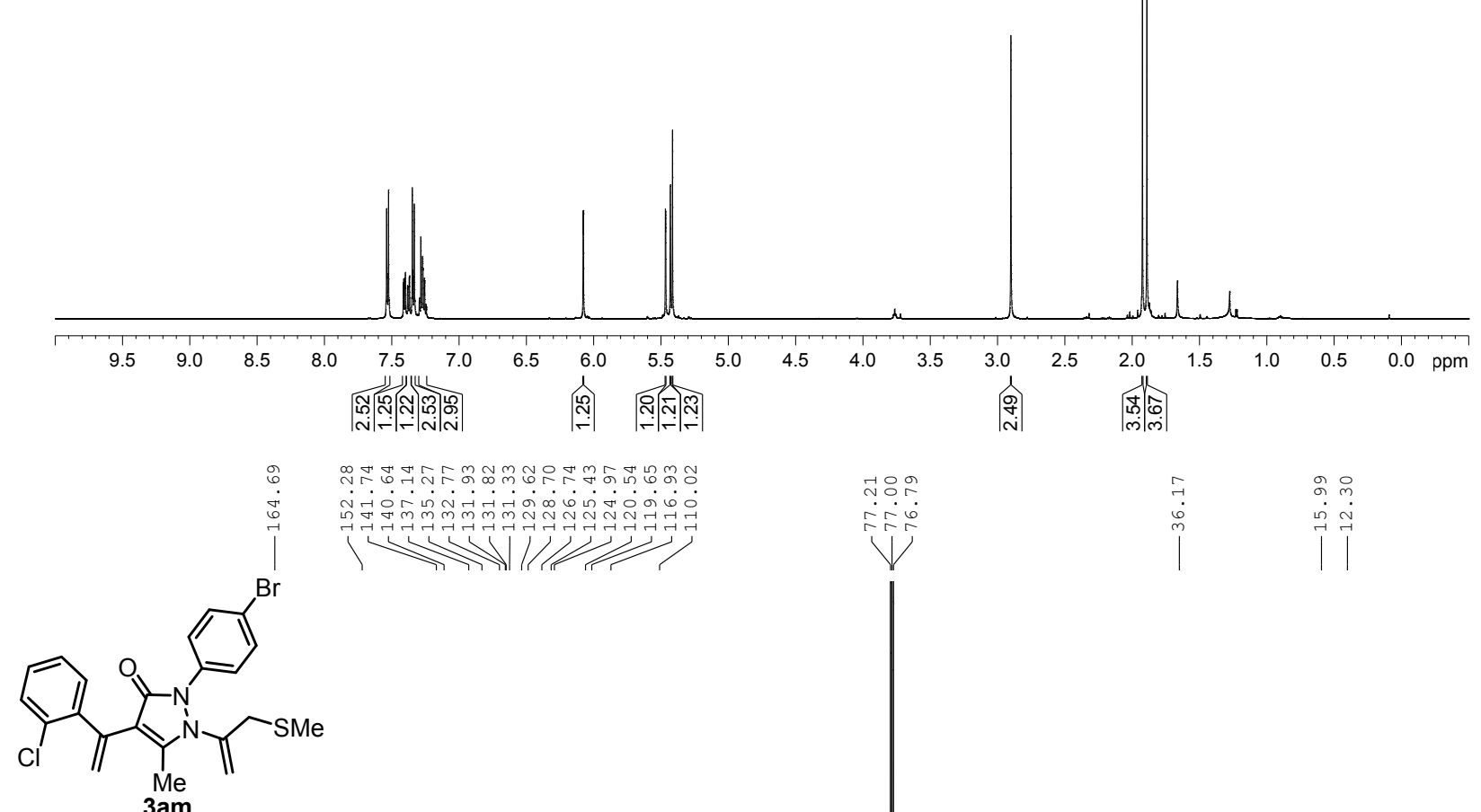

$\mathrm{CDCl}_{3}, 150 \mathrm{MHz}$

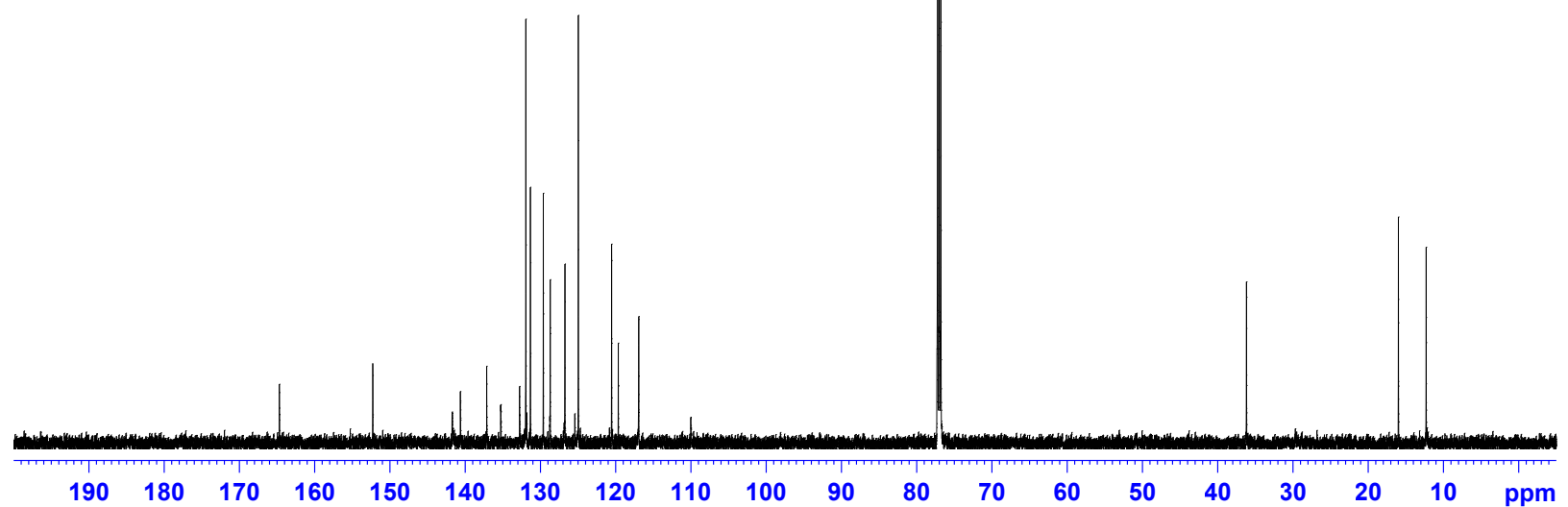



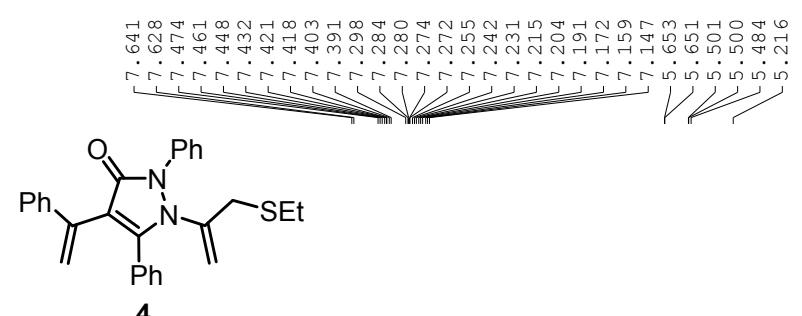

4

$\mathrm{CDCl}_{3}, 600 \mathrm{MHz}$
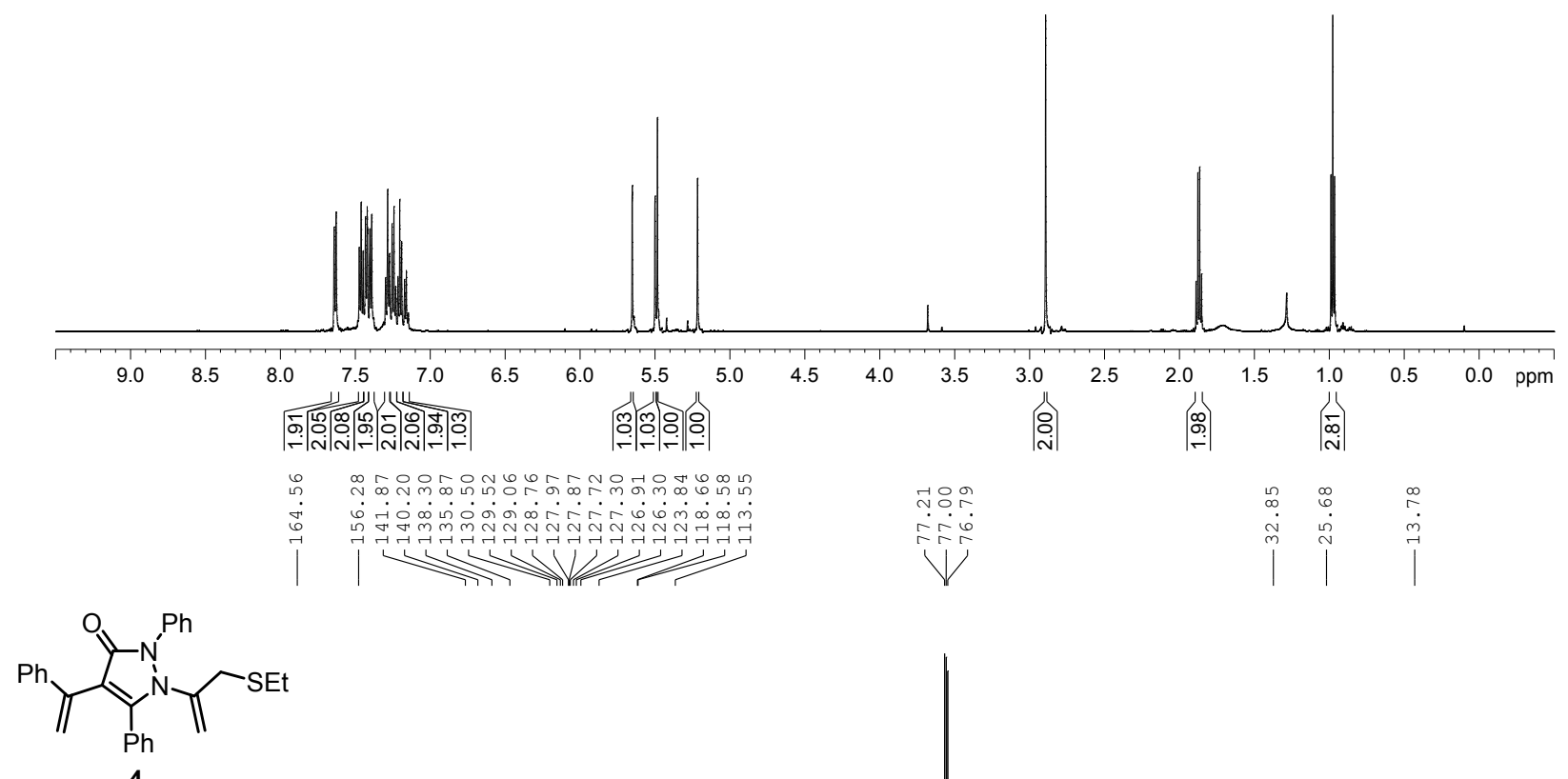

$\mathrm{CDCl}_{3}, 150 \mathrm{MHz}$

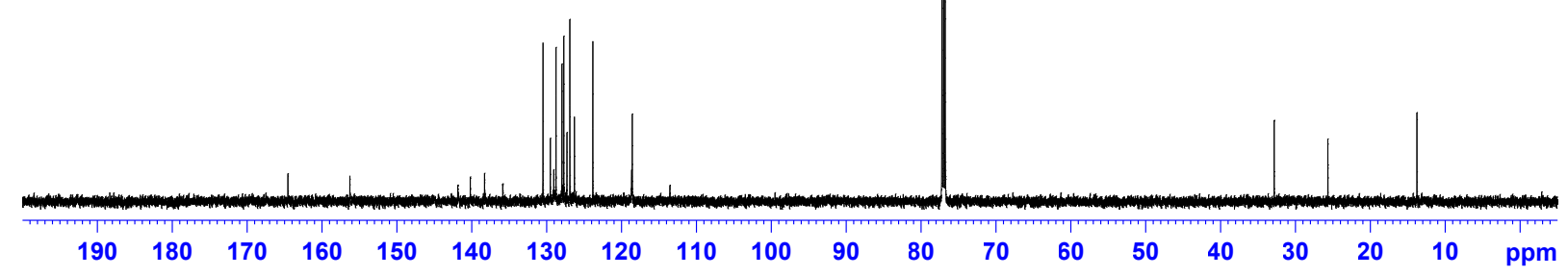



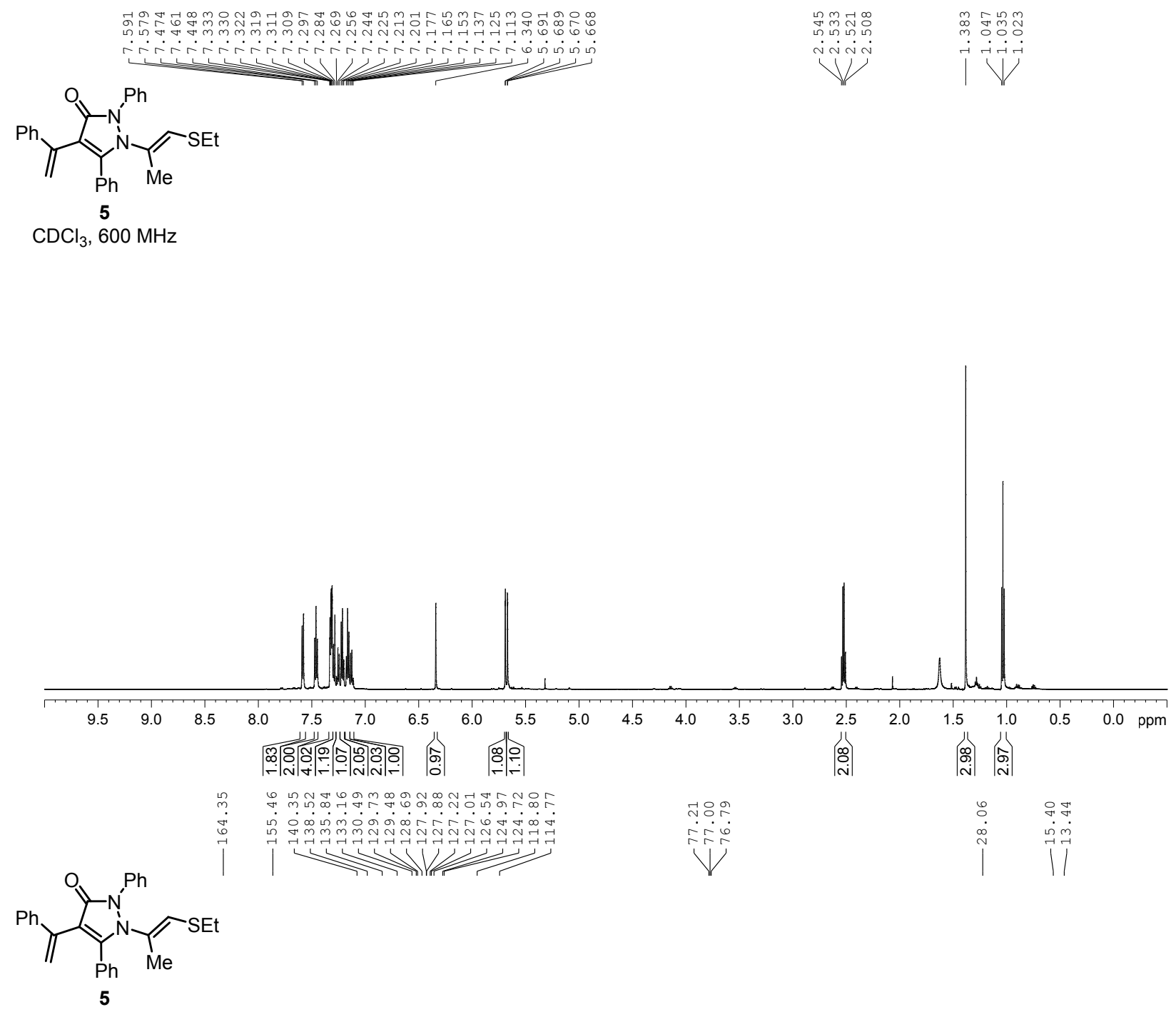

$\mathrm{CDCl}_{3}, 150 \mathrm{MHz}$

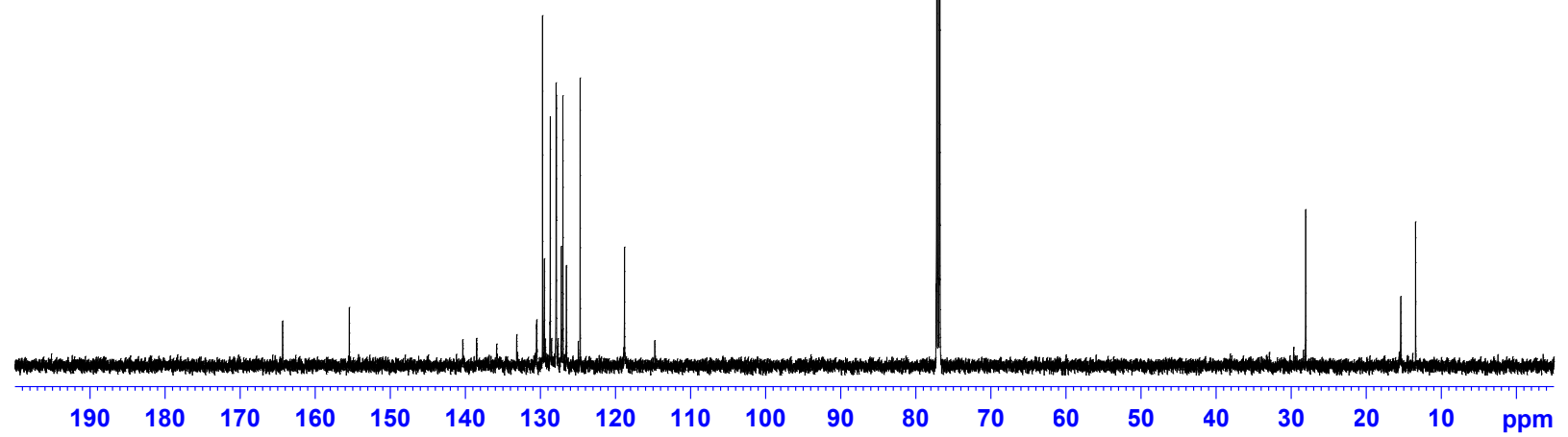




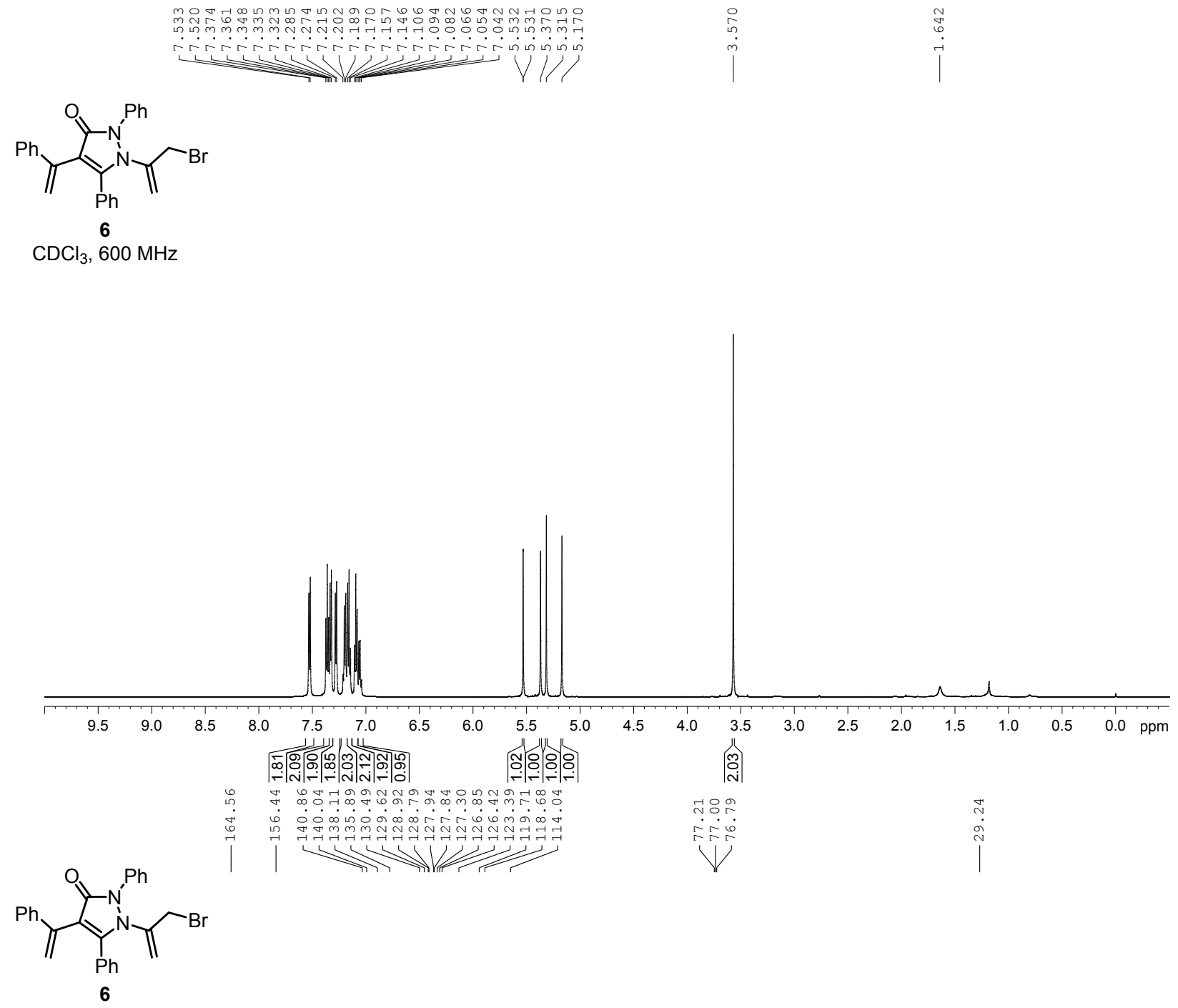

$\mathrm{CDCl}_{3}, 150 \mathrm{MHz}$

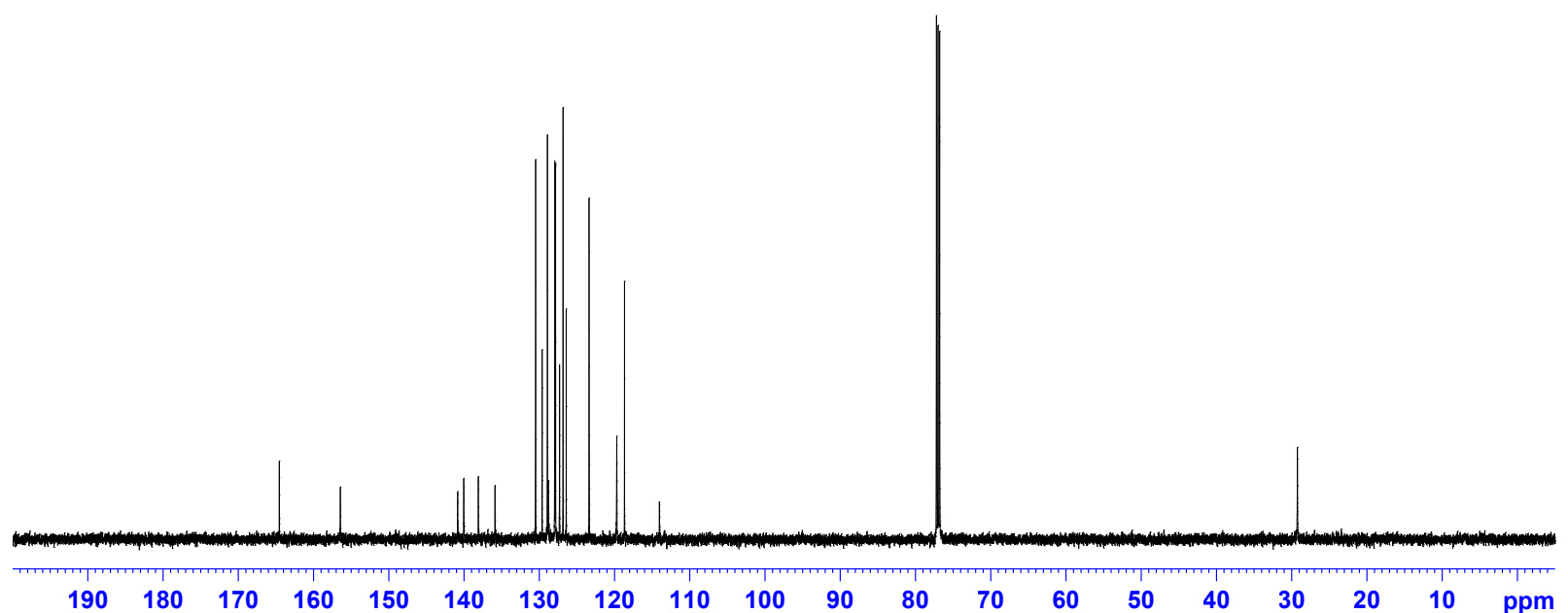




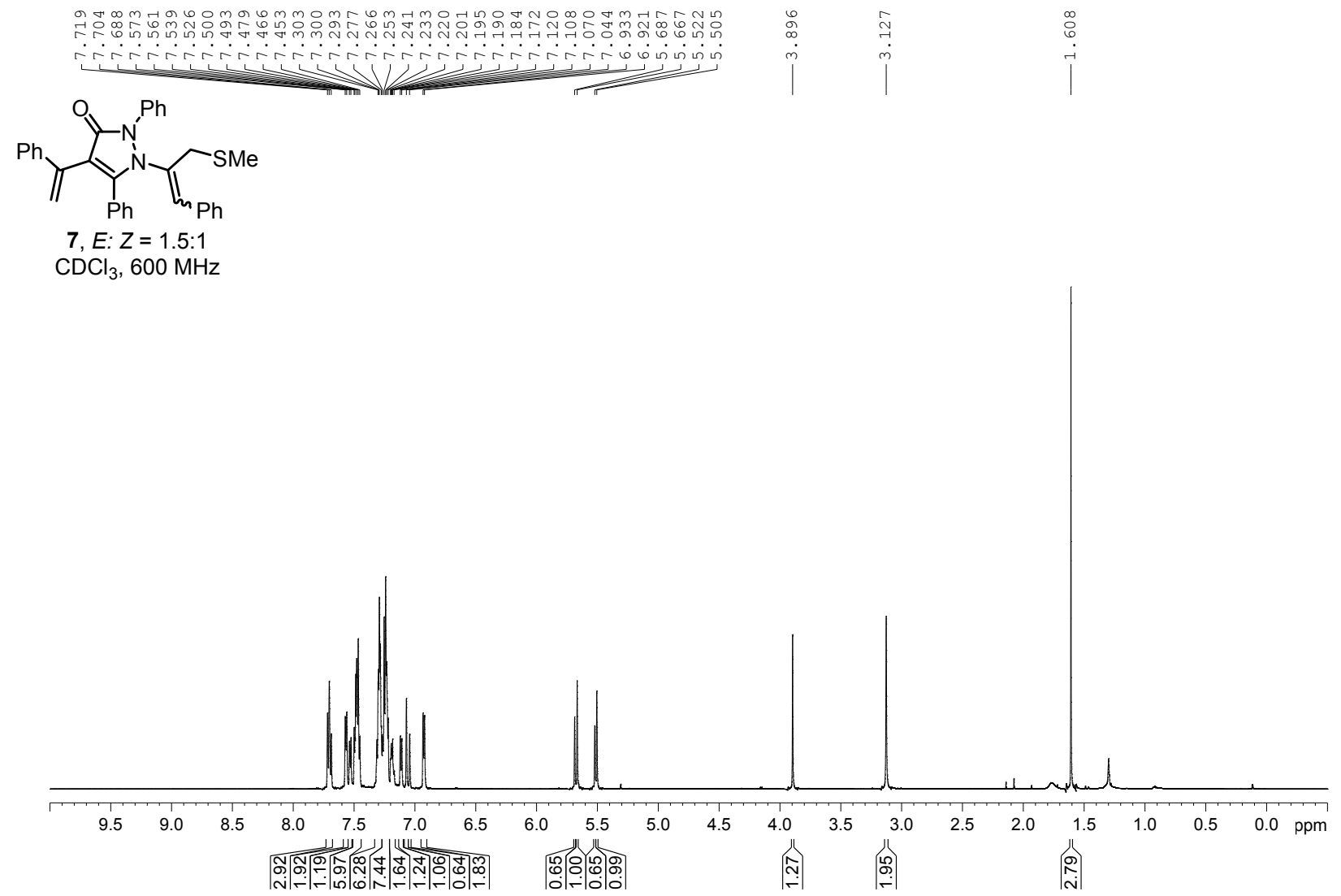

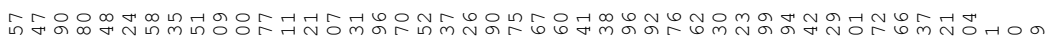

বुष

年

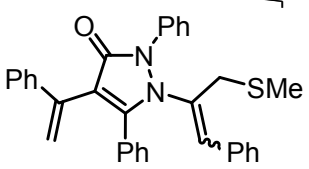

$7, E \cdot Z=1.5: 1$

$\mathrm{CDCl}_{3}, 150 \mathrm{MHz}$

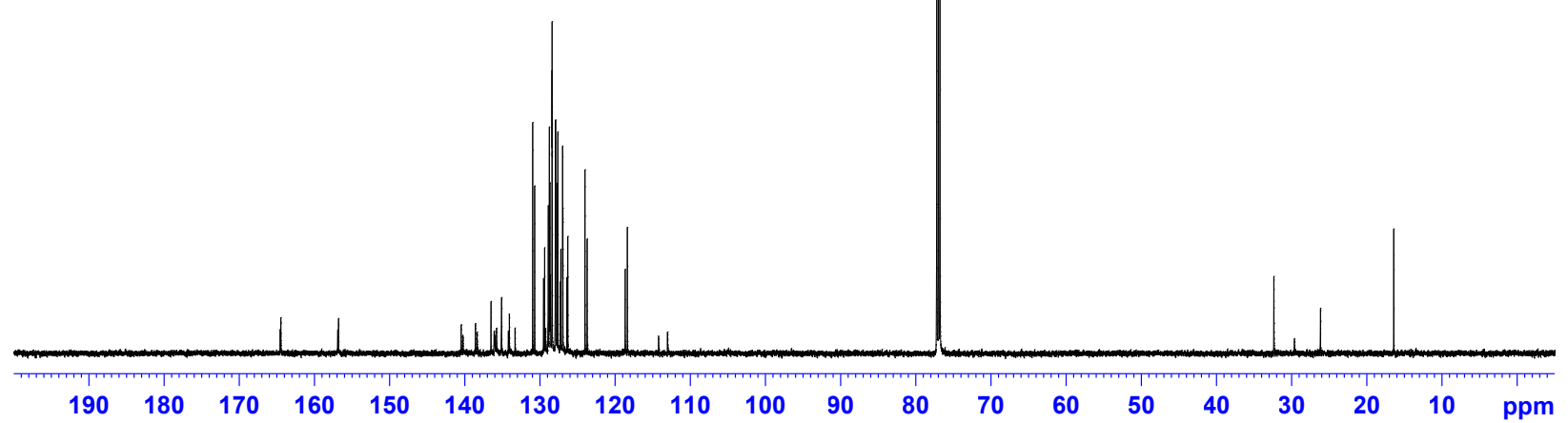




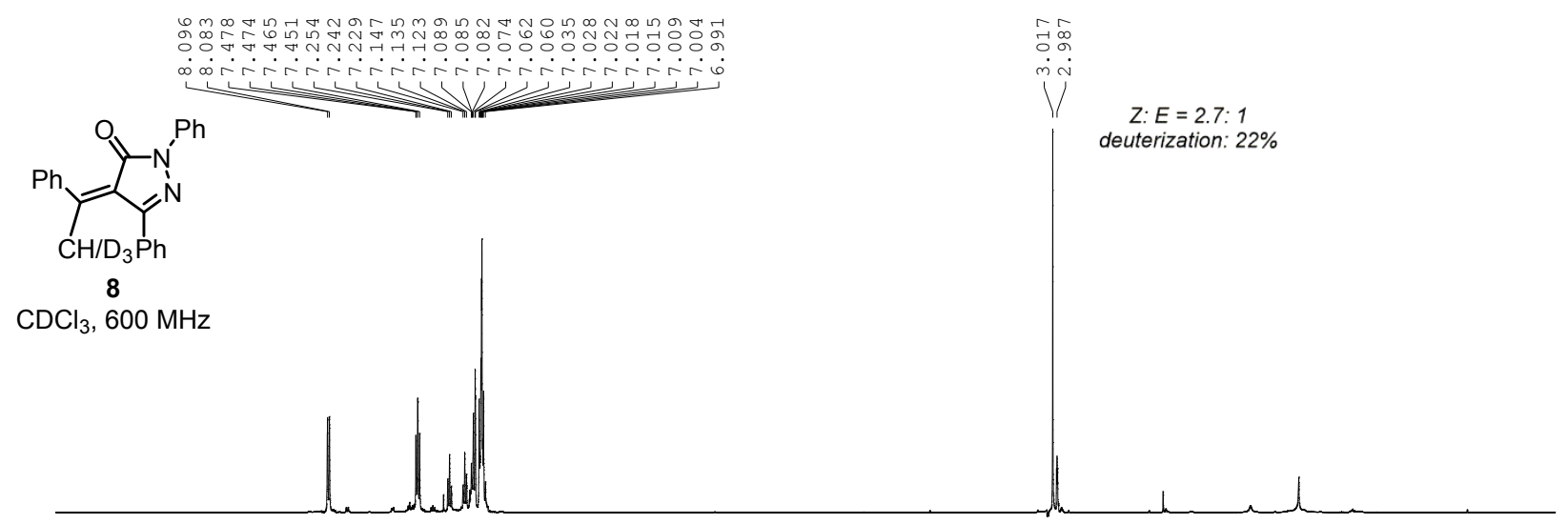

\begin{tabular}{|c|c|c|c|c|c|c|c|c|c|c|c|c|c|c|c|c|c|c|c|}
\hline 9.5 & 9.0 & 8.5 & $\left|\begin{array}{l}8.0 \\
N \\
F\end{array}\right|$ & $\begin{array}{l}7.5 \\
\text { o. } \\
\text { î }\end{array}$ & 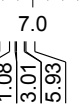 & 6.5 & 6.0 & 5.5 & 5.0 & 4.5 & 4.0 & 3.5 & 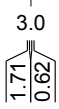 & 2.5 & 2.0 & 1.5 & 1.0 & 0.5 & 0.0 \\
\hline
\end{tabular}

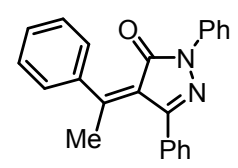

$1 a$

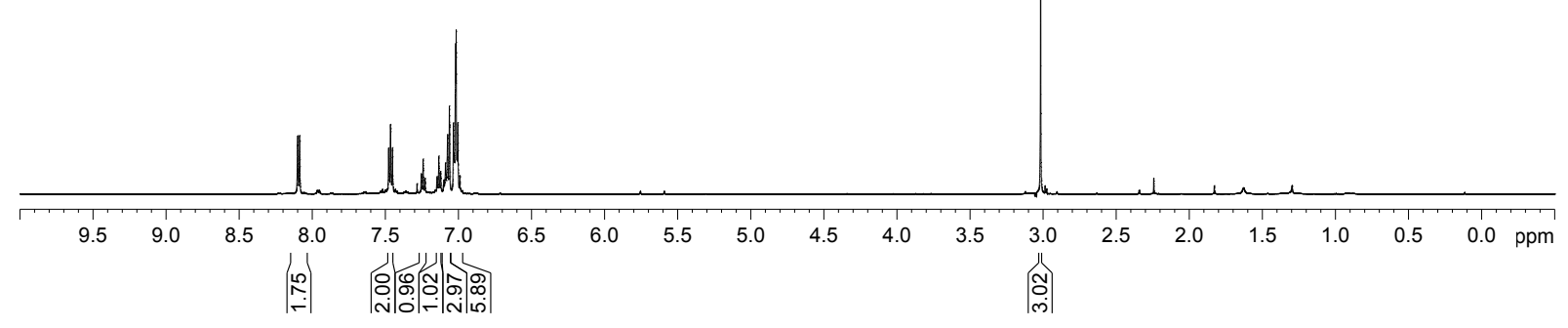



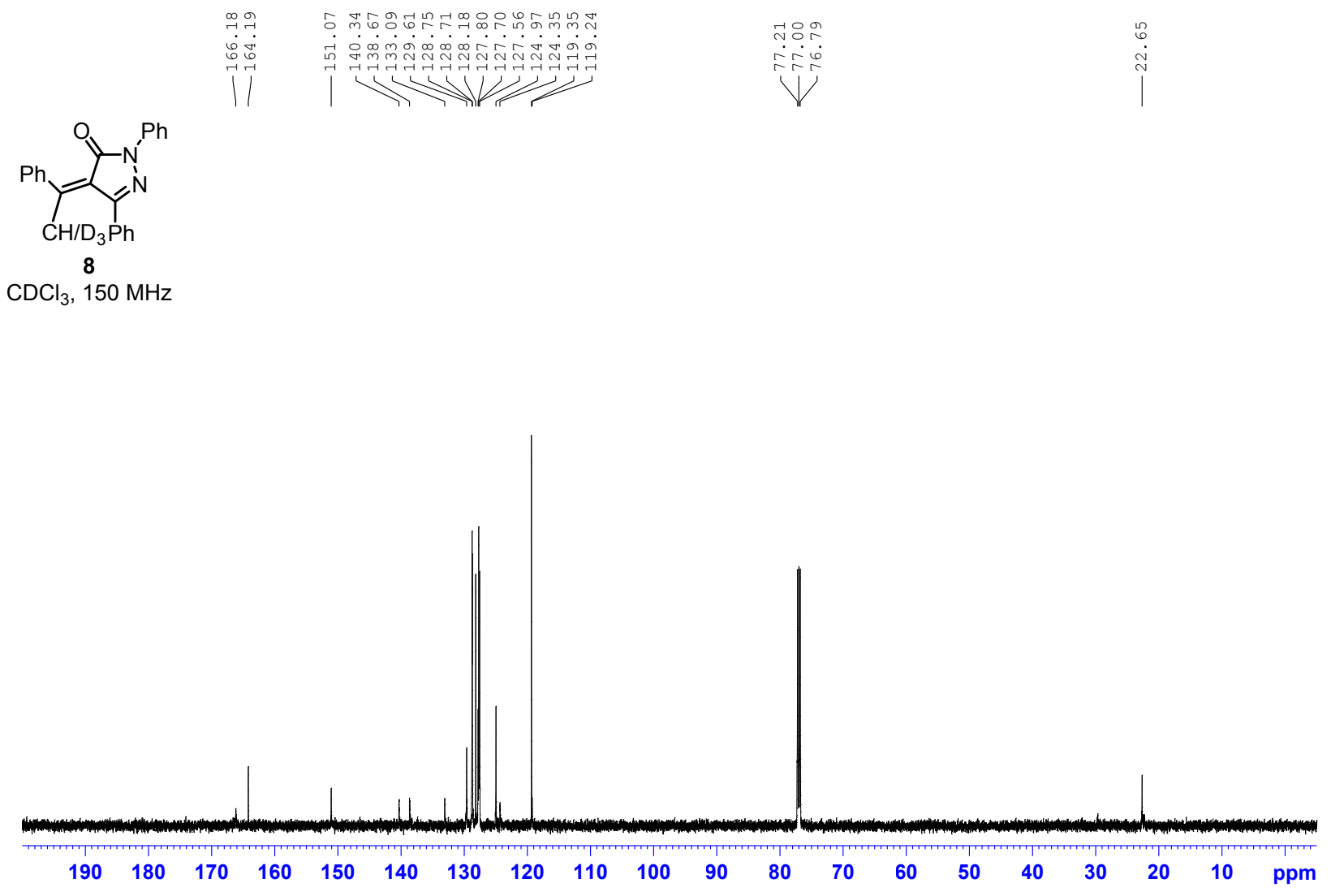


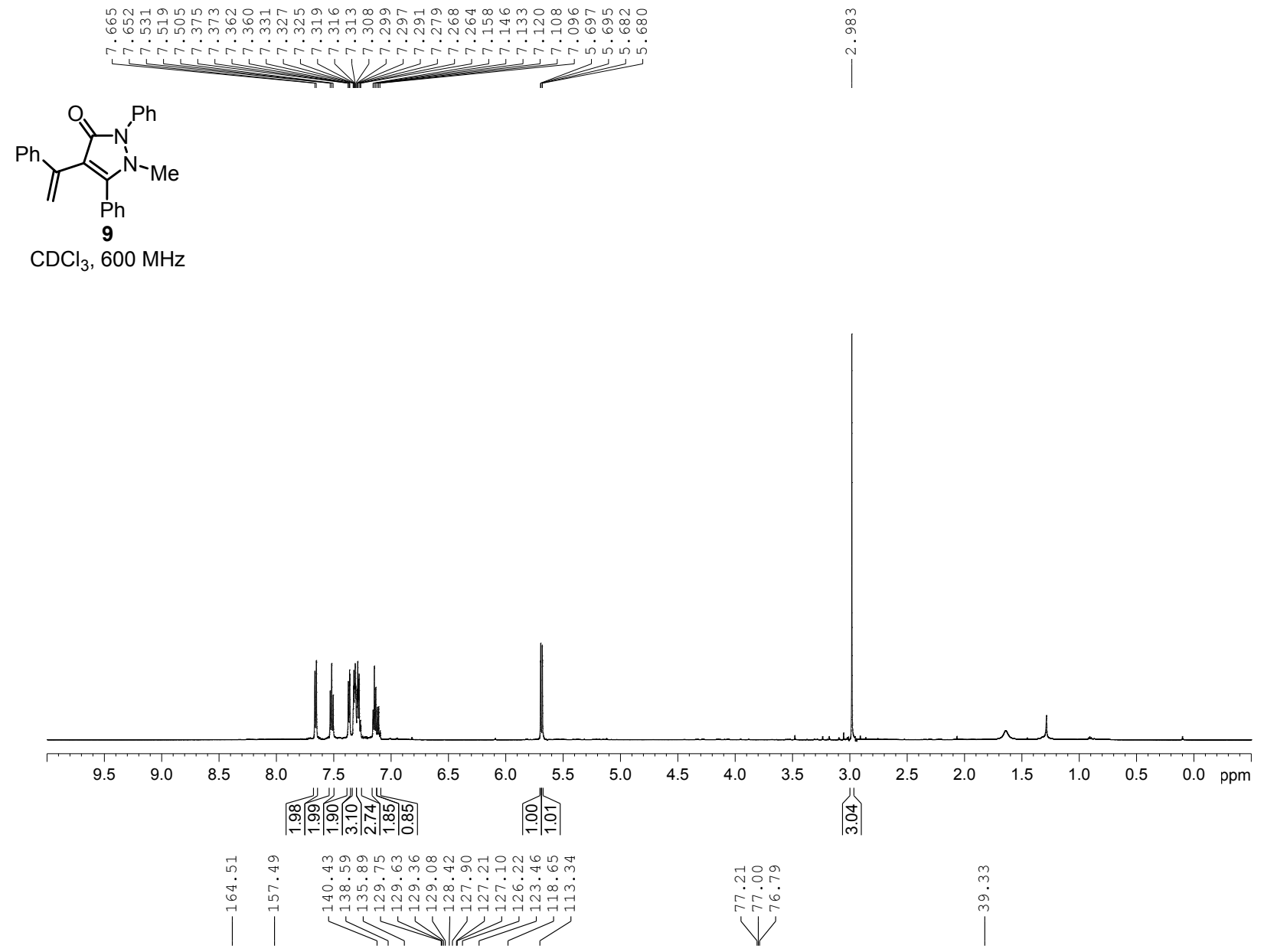<smiles>C=C(c1ccccc1)c1c(-c2ccccc2)n(C)c(=O)n1-c1ccccc1</smiles>

$\mathrm{CDCl}_{3}, 150 \mathrm{MHz}$

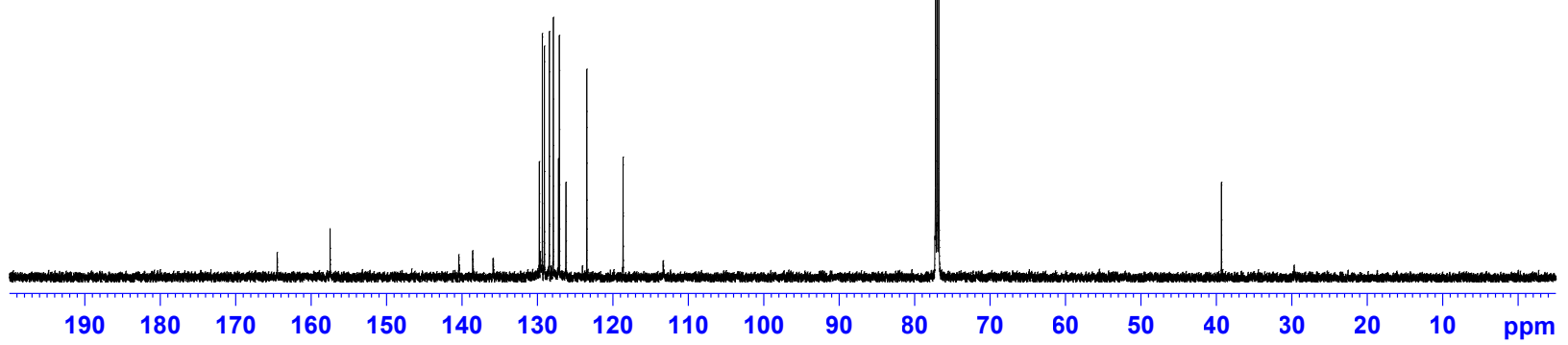

